\title{
A closer look at the $R_{D}$ and $R_{D^{*}}$ anomalies
}

\section{Debjyoti Bardhan, ${ }^{a}$ Pritibhajan Byakti $^{b}$ and Diptimoy Ghosh ${ }^{c}$}

\author{
${ }^{a}$ Department of Theoretical Physics, Tata Institute of Fundamental Research, \\ 1 Homi Bhabha Road, Mumbai 400005, India \\ ${ }^{b}$ Department of Theoretical Physics, Indian Association for the Cultivation of Science, \\ $2 A \&$ 2B, Raja S.C. Mullick Road, Jadavpur, Kolkata 700 032, India \\ ${ }^{c}$ Department of Particle Physics and Astrophysics, Weizmann Institute of Science, \\ Rehovot 76100, Israel \\ E-mail: debjyoti@theory.tifr.res.in, tppb@iacs.res.in, \\ diptimoy.ghosh@weizmann.ac.il
}

AbstRACT: The measurement of $R_{D}\left(R_{D^{*}}\right)$, the ratio of the branching fraction of $\bar{B} \rightarrow D \tau \bar{\nu}_{\tau}\left(\bar{B} \rightarrow D^{*} \tau \bar{\nu}_{\tau}\right)$ to that of $\bar{B} \rightarrow D l \bar{\nu}_{l}\left(\bar{B} \rightarrow D^{*} l \bar{\nu}_{l}\right)$, shows $1.9 \sigma(3.3 \sigma)$ deviation from its Standard Model (SM) prediction. The combined deviation is at the level of $4 \sigma$ according to the Heavy Flavour Averaging Group (HFAG). In this paper, we perform an effective field theory analysis (at the dimension 6 level) of these potential New Physics $(\mathrm{NP})$ signals assuming $\mathrm{SU}(3)_{\mathrm{C}} \times \mathrm{SU}(2)_{\mathrm{L}} \times \mathrm{U}(1)_{\mathrm{Y}}$ gauge invariance. We first show that, in general, $R_{D}$ and $R_{D^{*}}$ are theoretically independent observables and hence, their theoretical predictions are not correlated. We identify the operators that can explain the experimental measurements of $R_{D}$ and $R_{D^{*}}$ individually and also together. Motivated by the recent measurement of the $\tau$ polarisation in $\bar{B} \rightarrow D^{*} \tau \bar{\nu}_{\tau}$ decay, $P_{\tau}\left(D^{*}\right)$ by the Belle collaboration, we study the impact of a more precise measurement of $P_{\tau}\left(D^{*}\right)$ (and a measurement of $\left.P_{\tau}(D)\right)$ on the various possible NP explanations. Furthermore, we show that the measurement of $R_{D^{*}}$ in bins of $q^{2}$, the square of the invariant mass of the lepton-neutrino system, along with the information on $\tau$ polarisation and the forward-backward asymmetry of the $\tau$ lepton, can completely distinguish the various operator structures. We also provide the full expressions of the double differential decay widths for the individual $\tau$ helicities in the presence of all the 10 dimension- 6 operators that can contribute to these decays.

Keywords: Beyond Standard Model, Heavy Quark Physics

ARXIV EPRINT: 1610.03038 


\section{Contents}

1 Introduction 1

2 Operator basis 3

3 Observables 5

$4 \quad \bar{B} \rightarrow D$ form factors $\quad 6$

$5 \quad \bar{B} \rightarrow D^{*}$ form factors $\quad 9$

6 Expressions for $a_{\ell}^{D}, b_{\ell}^{D}$ and $c_{\ell}^{D}$ for $\bar{B} \rightarrow D \ell \bar{\nu}_{\ell} \quad 11$

7 Expressions for $a_{\ell}^{D^{*}}, b_{\ell}^{D^{*}}$ and $c_{\ell}^{D^{*}}$ for $\bar{B} \rightarrow D^{*} \ell \bar{\nu}_{\ell}$

8 Results 14

8.1 Explaining $R_{D}$ alone 14

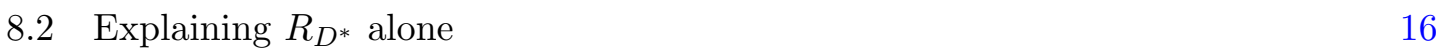

$\begin{array}{lll}8.3 & \text { Explaining } R_{D} \text { and } R_{D^{*}} \text { together } & 19\end{array}$

9 Summary 20

A Full expressions for $a_{\ell}^{D}, b_{\ell}^{D}$ and $c_{\ell}^{D} \quad 21$

B Full expressions for $a_{\ell}^{D^{*}}, b_{\ell}^{D^{*}}$ and $c_{\ell}^{D^{*}} \quad 22$

C Contribution of the Tensor operator $\mathcal{O}_{\text {TL }}^{c b \ell} \quad 27$

$\begin{array}{ll}\text { C.1 } \bar{B} \rightarrow D \tau \bar{\nu}_{\tau} & 27\end{array}$

C.2 $\bar{B} \rightarrow D^{*} \tau \bar{\nu}_{\tau}$

$\mathrm{D} \mathrm{SU}(3)_{\mathrm{C}} \times \mathrm{SU}(2)_{\mathrm{L}} \times \mathrm{U}(1)_{\mathrm{Y}}$ gauge invariance $\quad 28$

E RG running of Wilson coefficients 29

\section{Introduction}

In recent years, a number of experimental measurements involving $B$ meson decays have shown interesting deviations from their Standard Model (SM) expectations. Deviations have been seen both in the neutral current $b \rightarrow s$ decays $[1,2]^{1}$ as well as the charged current $b \rightarrow c$ processes. The most statistically significant deviation, at the $4 \sigma$ level [17], is seen in the combination of $R_{D}$ and $R_{D^{*}}$ which are defined as,

$$
R_{D^{(*)}}=\frac{\mathcal{B}\left(\bar{B} \rightarrow D^{(*)} \tau \bar{\nu}_{\tau}\right)}{\mathcal{B}\left(\bar{B} \rightarrow D^{(*)} l \bar{\nu}_{l}\right)}
$$

where $l=e$ or $\mu$. In table 1 , we collect all the relevant experimental results related to the $\bar{B} \rightarrow D^{(*)} \ell \nu_{\ell}$ decay processes.

\footnotetext{
${ }^{1}$ For theoretical implications, see for example [3-16] and the references therein.
} 


\begin{tabular}{|c|c|c|c|}
\hline \multicolumn{4}{|c|}{ List of Observables } \\
\hline \multirow{2}{*}{ Observable } & \multicolumn{2}{|r|}{ Experimental Results } & \multirow{2}{*}{ SM Prediction } \\
\hline & Experiment & Measured value & \\
\hline \multirow{3}{*}{$R_{D}$} & Belle & $0.375 \pm 0.064 \pm 0.026$ & \multirow{3}{*}{$\begin{array}{c}0.299 \pm 0.011[19] \\
0.300 \pm 0.008[22] \\
0.299 \pm 0.003[23] \\
\mathbf{0 . 3 0 0} \pm \mathbf{0 . 0 1 1}\end{array}$} \\
\hline & BaBar & $0.440 \pm 0.058 \pm 0.042 \quad[20,21]$ & \\
\hline & HFAG average & $0.397 \pm 0.040 \pm 0.028$ & \\
\hline \multirow{7}{*}{$R_{D^{*}}$} & Belle & $0.293 \pm 0.038 \pm 0.015 \quad[18]$ & \multirow{7}{*}{$\begin{array}{c}0.252 \pm 0.003[24] \\
\mathbf{0 . 2 5 4} \pm \mathbf{0 . 0 0 4}\end{array}$} \\
\hline & Belle & $0.302 \pm 0.030 \pm 0.011$ & \\
\hline & BaBar & $0.332 \pm 0.024 \pm 0.018 \quad[20,21]$ & \\
\hline & $\mathrm{LHCb}$ & $0.336 \pm 0.027 \pm 0.030 \quad[26]$ & \\
\hline & HFAG average & $0.316 \pm 0.016 \pm 0.010 \quad[17]$ & \\
\hline & Belle & $0.276 \pm 0.034_{-0.026}^{+0.029} \quad[27]$ & \\
\hline & Our average & $0.310 \pm 0.017$ & \\
\hline $\mathcal{B}\left(\bar{B} \rightarrow D \tau \bar{\nu}_{\tau}\right)$ & BaBar & $1.02 \pm 0.13 \pm 0.11 \% \quad[20]$ & $0.633 \pm 0.014 \%$ \\
\hline $\mathcal{B}\left(\bar{B} \rightarrow D^{*} \tau \bar{\nu}_{\tau}\right)$ & BaBar & $1.76 \pm 0.13 \pm 0.12 \% \quad[20]$ & $1.28 \pm 0.09 \%$ \\
\hline $\mathcal{B}\left(\bar{B} \rightarrow D l \bar{\nu}_{l}\right)$ & HFAG average & $2.13 \pm 0.03 \pm 0.09 \% \quad[17]$ & $2.11_{-0.10}^{+0.12} \%$ \\
\hline $\mathcal{B}\left(\bar{B} \rightarrow D^{*} l \bar{\nu}_{l}\right)$ & HFAG average & $4.93 \pm 0.01 \pm 0.11 \% \quad[17]$ & $5.04_{-0.42}^{+0.44} \%$ \\
\hline$P_{\tau}\left(\bar{B} \rightarrow D \tau \bar{\nu}_{\tau}\right)$ & & & $\begin{array}{c}0.325 \pm 0.009[28] \\
\mathbf{0 . 3 2 5} \pm \mathbf{0 . 0 1 2}\end{array}$ \\
\hline$P_{\tau}\left(\bar{B} \rightarrow D^{*} \tau \bar{\nu}_{\tau}\right)$ & Belle & $-0.44 \pm 0.47_{-0.17}^{+0.20}$ & $\begin{array}{c}-0.497 \pm 0.013[27,29] \\
-\mathbf{0 . 4 9 7} \pm \mathbf{0 . 0 0 8}\end{array}$ \\
\hline $\mathcal{A}_{F B}^{D}$ & & & $-0.360_{-0.001}^{+0.002}$ \\
\hline $\mathcal{A}_{F B}^{D^{*}}$ & & & $0.064 \pm 0.014$ \\
\hline
\end{tabular}

Table 1. The relevant observables, their experimental measurements and the SM predictions are shown. While computing the branching ratios, we have used $V_{c b}=0.04$. As HFAG has not yet included the latest Belle measurement of $R_{D^{*}}$ in their global average, we have taken a naive weighted average of the latest Belle result and the average given by HFAG. However, since the recent Belle result has a large uncertainty, it does not affect the previous world average in any significant way. The values given in boldface are our results for the SM predictions. Note that, for the $\bar{B} \rightarrow D^{*} \ell \bar{\nu}_{\ell}$ SM predictions, the uncertainties correspond to $2 \sigma$ uncertainties in the form factor parameters, see section 5 for more details.

Note that, we have used the notation $\ell$ to denote any lepton (e, $\mu$ or $\tau$ ) and $l$ to denote only the light leptons, e and $\mu$.

The large statistical significance of the anomaly in $R_{D}$ and $R_{D^{*}}$ has spurred a lot of interest in this decay modes in the last few years [24, 29-63] and various possible theoretical explanations have been proposed.

The main purpose of this work is to identify observables which can help distinguish the different NP Lorentz structures that can potentially solve the $R_{D}$ and $R_{D^{*}}$ anomalies. We first perform an operator analysis of these potential NP signals by considering all the dimension-6 operators that are consistent with SM gauge invariance. We compute the val- 
ues of the relevant Wilson coefficients (WCs) that explain the experimental measurements within their $1 \sigma$ ranges. It is important to note that we consider the presence of NP only in the tau-channel and not for the electron or the muon channels. Thus, in our calculations of $R_{D}$ and $R_{D^{*}}$, we use the SM values of the WCs in the denominator. For these values of the WCs, we compute the predictions for a few observables that have the potential to distinguish between the various NP operators. Although we provide numerical results only for the operators that are consistent with SM gauge invariance, we provide the analytical expressions for the double differential decay rates for the individual $\tau$ helicities for all the 10 independent dimension- 6 operators contributing to these decays. To our knowledge, we are the first in the literature to provide the full expressions.

As we show later, $R_{D}$ and $R_{D^{*}}$ are in general theoretically independent observables and the anomalies can exist independently. A future measurement might reveal a greater anomaly in one of them without affecting the other. Hence, in this paper, we attempt to explain each without worrying about the other initially, but then also point out how both can be explained together.

Very recently, the Belle collaboration reported the first measurement of the $\tau$-polarisation in the decay $\bar{B} \rightarrow D^{*} \tau \bar{\nu}_{\tau}[27]$. While the uncertainty in this measurement is rather large now, motivated by the possibility of more precise measurements in the future, we investigate how such a measurement can distinguish the various NP explanations of $R_{D}$ and $R_{D^{*}}$. Furthermore, we show that measurements of $R_{D^{*}}$ in bins of $q^{2}$ can provide important information about the nature of short distance physics. In fact, a combination of binwise $R_{D^{*}}$ and more precise measurements (that can be done in Belle II, for example) of $\tau$ polarisation in both the $\bar{B} \rightarrow D \tau \bar{\nu}_{\tau}$ and $\bar{B} \rightarrow D^{*} \tau \bar{\nu}_{\tau}$ decays can completely distinguish all the different NP operators. Moreover, we show that the forward-backward asymmetry of the $\tau$ lepton (in the $\tau-\nu_{\tau}$ rest frame) also has the potential to differentiate the various NP Lorentz structures.

The paper is organised as follows: in section 2 we write down all the operators relevant for this study and define the notations for the corresponding WCs. The various observables of our interest are defined in section 3. The sections 4 and 5 discuss the form factors required for the calculation of the decay amplitudes. The analytic expressions for the double differential decay widths for the individual lepton helicities are shown in sections 6 and 7. In the following section (section 8), we present all our numerical results. Finally, we summarise our findings in section 9.

The full expressions for the double differential decay widths are shown in the appendices $\mathrm{A}$ and $\mathrm{B}$, and the contribution of the tensor operator $\mathcal{O}_{\mathrm{TL}}$ is discussed in appendix $\mathrm{C}$. In appendix D, we show how our operators are related to the dimension-6 operators of [64]. The renormalisation group equations for the WCs are computed in appendix E.

\section{Operator basis}

The effective Lagrangian for the $b \rightarrow c \ell \bar{\nu}$ process at the dimension 6 level is given by,

$$
\begin{aligned}
\mathcal{L}_{\text {eff }}^{b \rightarrow c \ell \nu}=\frac{2 G_{F} V_{c b}}{\sqrt{2}} & \left(C_{9}^{c b \ell} \mathcal{O}_{9}^{c b \ell}+C_{9}^{c b \ell^{\prime}} \mathcal{O}_{9}^{c b \ell^{\prime}}+C_{10}^{c b \ell} \mathcal{O}_{10}^{c b \ell}+C_{10}^{c b \ell^{\prime}} \mathcal{O}_{10}^{c b \ell^{\prime}}+C_{s}^{c b \ell} \mathcal{O}_{s}^{c b \ell}+C_{s}^{c b \ell^{\prime}} \mathcal{O}_{s}^{c b \ell^{\prime}}\right. \\
& \left.+C_{p}^{c b \ell} \mathcal{O}_{p}^{c b \ell}+C_{p}^{c b \ell^{\prime}} \mathcal{O}_{p}^{c b \ell^{\prime}}+C_{T}^{c b \ell} \mathcal{O}_{T}^{c b \ell}+C_{T 5}^{c b \ell} \mathcal{O}_{T 5}^{c b \ell}\right)
\end{aligned}
$$


where $\mathcal{O}_{i}^{c b \ell}$ constitute a complete basis of 6 -dimensional operators and $C_{i}^{\text {cbl }}$ are the corresponding Wilson coefficients defined at the renormalization scale $\mu=m_{b}$. In the SM, $C_{9}^{c b \ell}=-C_{10}^{c b \ell}=1$ and all the other WCs vanish. The full set of operators is given by:

$$
\begin{aligned}
\mathcal{O}_{9}^{c b \ell} & =\left[\bar{c} \gamma^{\mu} \mathrm{P}_{L} b\right]\left[\bar{\ell} \gamma_{\mu} \nu\right] & \mathcal{O}_{9}^{c b \ell^{\prime}} & =\left[\bar{c} \gamma^{\mu} \mathrm{P}_{R} b\right]\left[\bar{\ell} \gamma_{\mu} \nu\right] \\
\mathcal{O}_{10}^{c b \ell} & =\left[\bar{c} \gamma^{\mu} \mathrm{P}_{L} b\right]\left[\bar{\ell} \gamma_{\mu} \gamma_{5} \nu\right] & \mathcal{O}_{10}^{c b \ell^{\prime}} & =\left[\bar{c} \gamma^{\mu} \mathrm{P}_{R} b\right]\left[\bar{\ell} \gamma_{\mu} \gamma_{5} \nu\right] \\
\mathcal{O}_{s}^{c b \ell} & =\left[\bar{c} \mathrm{P}_{L} b\right][\bar{\ell} \nu] & \mathcal{O}_{s}^{c b \ell^{\prime}} & =\left[\bar{c} \mathrm{P}_{R} b\right][\bar{\ell} \nu] \\
\mathcal{O}_{p}^{c b \ell} & =\left[\bar{c} \mathrm{P}_{L} b\right]\left[\left[\bar{\ell} \gamma_{5} \nu\right]\right. & \mathcal{O}_{p}^{c b \ell^{\prime}} & =\left[\bar{c} \mathrm{P}_{R} b\right]\left[\left[\bar{\ell} \gamma_{5} \nu\right]\right. \\
\mathcal{O}_{T}^{c b \ell} & =\left[\bar{c} \sigma^{\mu \nu} b\right]\left[\bar{\ell} \sigma_{\mu \nu} \nu\right] & \mathcal{O}_{T 5}^{c b \ell} & =\left[\bar{c} \sigma^{\mu \nu} b\right]\left[\bar{\ell} \sigma_{\mu \nu} \gamma_{5} \nu\right]
\end{aligned}
$$

The other possible tensor structures are related to $\mathcal{O}_{T}^{c b \ell}$ and $\mathcal{O}_{T 5}^{\text {cbl }}$ in the following way,

$$
\begin{aligned}
\epsilon_{\mu \nu \alpha \beta}\left[\bar{c} \sigma^{\mu \nu} b\right]\left[\bar{\ell} \sigma^{\alpha \beta} \nu\right] & =-2 i \mathcal{O}_{T 5}^{c b \ell} \\
{\left[\bar{c} \sigma^{\mu \nu} \gamma_{5} b\right]\left[\bar{\ell} \sigma_{\mu \nu} \gamma_{5} \nu\right] } & =\mathcal{O}_{T}^{c b \ell} \\
{\left[\bar{c} \sigma^{\mu \nu} \gamma_{5} b\right]\left[\bar{\ell} \sigma_{\mu \nu} \nu\right] } & =\mathcal{O}_{T 5}^{c b \ell} .
\end{aligned}
$$

Note that the above basis of operators is different from the one used in some earlier literature $[31,36]$. For example, the reference [31] uses the following set of operators,

$$
\begin{aligned}
\mathcal{O}_{\mathrm{VL}}^{c b \ell} & =\left[\bar{c} \gamma^{\mu} b\right]\left[\bar{\ell} \gamma_{\mu} P_{L} \nu\right] & \mathcal{O}_{\mathrm{VR}}^{c b \ell} & =\left[\bar{c} \gamma^{\mu} b\right]\left[\bar{\ell} \gamma_{\mu} P_{R} \nu\right] \\
\mathcal{O}_{\mathrm{AL}}^{c b \ell} & =\left[\bar{c} \gamma^{\mu} \gamma_{5} b\right]\left[\bar{\ell} \gamma_{\mu} P_{L} \nu\right] & \mathcal{O}_{\mathrm{AR}}^{c b \ell} & =\left[\bar{c} \gamma^{\mu} \gamma_{5} b\right]\left[\bar{\ell} \gamma_{\mu} P_{R} \nu\right] \\
\mathcal{O}_{\mathrm{SL}}^{c b \ell} & =[\bar{c} b]\left[\bar{\ell} P_{L} \nu\right] & & \mathcal{O}_{\mathrm{SR}}^{c b \ell}=[\bar{c} b]\left[\bar{\ell} P_{R} \nu\right] \\
\mathcal{O}_{\mathrm{PL}}^{c b \ell} & =\left[\bar{c} \gamma_{5} b\right]\left[\left[\bar{\ell} P_{L} \nu\right]\right. & \mathcal{O}_{\mathrm{PR}}^{c c \ell} & =\left[\bar{c} \gamma_{5} b\right]\left[\left[\bar{\ell} P_{R} \nu\right]\right. \\
\mathcal{O}_{\mathrm{TL}}^{c b \ell} & =\left[\bar{c} \sigma^{\mu \nu} b\right]\left[\bar{\ell} \sigma_{\mu \nu} P_{L} \nu\right] & \mathcal{O}_{\mathrm{TR}}^{c b \ell} & =\left[\bar{c} \sigma^{\mu \nu} b\right]\left[\bar{\ell} \sigma_{\mu \nu} P_{R} \nu\right]
\end{aligned}
$$

The Wilson coefficients of these two basis of operators are related through the following equations,

$$
\begin{array}{rlrl}
C_{\mathrm{VL}}^{c b \ell} & =\frac{1}{2}\left(C_{9}^{c b \ell}-C_{10}^{c b \ell}+C_{9}^{c b \ell^{\prime}}-C_{10}^{c b \ell^{\prime}}\right) & C_{\mathrm{SR}}^{c b \ell} & =\frac{1}{2}\left(C_{s}^{c b \ell}+C_{p}^{c b \ell}+C_{s}^{c b \ell^{\prime}}+C_{p}^{c b \ell^{\prime}}\right) \\
C_{\mathrm{AL}}^{c b \ell} & =\frac{1}{2}\left(-C_{9}^{c b \ell}+C_{10}^{c b \ell}+C_{9}^{c b \ell^{\prime}}-C_{10}^{c b \ell^{\prime}}\right) & C_{\mathrm{PR}}^{c b \ell} & =\frac{1}{2}\left(-C_{s}^{c b \ell}-C_{p}^{c b \ell}+C_{s}^{c b \ell^{\prime}}+C_{p}^{c b \ell^{\prime}}\right) \\
C_{\mathrm{SL}}^{c b \ell} & =\frac{1}{2}\left(C_{s}^{c b \ell}-C_{p}^{c b \ell}+C_{s}^{c b \ell^{\prime}}-C_{p}^{c b \ell^{\prime}}\right) & & C_{\mathrm{VR}}^{c b \ell}=\frac{1}{2}\left(C_{9}^{c b \ell}+C_{10}^{c b \ell}+C_{9}^{c b \ell^{\prime}}+C_{10}^{c b \ell^{\prime}}\right) \\
C_{\mathrm{PL}}^{c b \ell}=\frac{1}{2}\left(-C_{s}^{c b \ell}+C_{p}^{c b \ell}+C_{s}^{c b \ell^{\prime}}-C_{p}^{c b \ell^{\prime}}\right) & C_{\mathrm{AR}}^{c b \ell}=\frac{1}{2}\left(-C_{9}^{c b \ell}-C_{10}^{c b \ell}+C_{9}^{c b \ell^{\prime}}+C_{10}^{c b \ell^{\prime}}\right) \\
C_{\mathrm{TL}}^{c b \ell}=\left(C_{T}^{c b \ell}-C_{T 5}^{c b \ell}\right) & C_{\mathrm{TR}}^{c b \ell}=\left(C_{T}^{c b \ell}+C_{T 5}^{c b \ell}\right)
\end{array}
$$


We now assume the neutrino in the final state to be left handed. This implies that the WCs in eq. (2.1) satisfy the following relations,

$$
\begin{aligned}
C_{9}^{c b \ell} & =-C_{10}^{c b \ell} \\
C_{9}^{c b \ell^{\prime}} & =-C_{10}^{c b \ell^{\prime}} \\
C_{s}^{c b \ell} & =-C_{p}^{c b \ell} \\
C_{s}^{c b \ell^{\prime}} & =-C_{p}^{c b \ell^{\prime}} \\
C_{T}^{c b \ell} & =-C_{T 5}^{c b \ell} .
\end{aligned}
$$

Consequently, all the WCs in the right hand column of eq. (2.8) vanish. Note that, the operators on the left hand column of eq. (2.8) are the only ones that are consistent with the full gauge invariance of the SM. In appendix D, we show how these WCs are related to the 6-dimensional operators listed in [64]. Moreover, since many microscopic models do not generate the tensor operator, we neglect them in the main text and study its effect only in the appendix (see appendix C).

Although, we do not study the effects of the operators with a right handed neutrino (the ones in the right hand column of eq. (2.8)), we compute the full analytic expressions considering all the 10 operators for the first time in the literature. The results are presented in appendices $\mathrm{A}$ and $\mathrm{B}$.

\section{Observables}

The double differential branching fractions for the decays $\bar{B} \rightarrow D \ell \bar{\nu}_{\ell}$ and $\bar{B} \rightarrow D^{*} \ell \bar{\nu}_{\ell}$ can be written as

$$
\frac{d^{2} \mathcal{B}_{\ell}^{D^{(*)}}}{d q^{2} d(\cos \theta)}=\mathcal{N}\left|p_{D^{(*)}}\right|\left(a_{\ell}^{D^{(*)}}+b_{\ell}^{D^{(*)}} \cos \theta+c_{\ell}^{D^{(*)}} \cos ^{2} \theta\right)
$$

The normalisation factor, $\mathcal{N}$ and the absolute value of the $D^{(*)}$-meson momentum, $\left|p_{D^{(*)}}\right|$ are given by,

$$
\begin{aligned}
\mathcal{N} & =\frac{\tau_{B} G_{F}^{2}\left|V_{c b}\right|^{2} q^{2}}{256 \pi^{3} M_{B}^{2}}\left(1-\frac{m_{\ell}^{2}}{q^{2}}\right)^{2} \\
\left|p_{D^{(*)}}\right| & =\frac{\sqrt{\lambda\left(M_{B}^{2}, M_{D^{(*)}}^{2}, q^{2}\right)}}{2 M_{B}}
\end{aligned}
$$

where $\lambda(a, b, c)=a^{2}+b^{2}+c^{2}-2(a b+b c+c a)$. The angle $\theta$ is defined as the angle between the lepton and $D^{(*)}$-meson in the lepton-neutrino centre-of-mass frame, and $q^{2}$ is the invariant mass squared of the lepton-neutrino system.

The total branching fraction is given by,

$$
\mathcal{B}_{\ell}^{D^{(*)}}=\int \mathcal{N}\left|p_{D^{(*)}}\right|\left(2 a_{\ell}^{D^{(*)}}+\frac{2}{3} c_{\ell}^{D^{(*)}}\right) d q^{2}
$$


The observables $R_{D}$ and $R_{D^{*}}$ have already been defined in eq. (1.1). We now define binned $R_{D^{(*)}}$ in the following way,

$$
R_{D^{(*)}}\left[q^{2} \text { bin }\right]=\frac{\mathcal{B}_{\tau}^{D^{(*)}}\left[q^{2} \text { bin }\right]}{\mathcal{B}_{l}^{D^{(*)}}\left[q^{2} \text { bin }\right]}
$$

For the decays with $\tau$ lepton in the final state, the polarisation of the $\tau$ also constitutes an useful observable and can potentially be used to distinguish the NP Lorentz structures. The $\tau$ polarisation fraction is defined in the following way,

$$
P_{\tau}\left(D^{(*)}\right)=\frac{\Gamma_{\tau}^{D^{(*)}}(+)-\Gamma_{\tau}^{D^{(*)}}(-)}{\Gamma_{\tau}^{D^{(*)}}(+)+\Gamma_{\tau}^{D^{(*)}}(-)}
$$

where, $\Gamma_{\tau}^{D^{(*)}}(+)$ and $\Gamma_{\tau}^{D^{(*)}}(-)$ are the decay widths for positive and negative helicity $\tau$ leptons respectively.

The $\tau$ forward-backward asymmetry, $\mathcal{A}_{F B}^{D^{(*)}}$ is defined as

$$
\begin{aligned}
\mathcal{A}_{F B}^{D^{(*)}} & =\frac{\int_{0}^{\pi / 2} \frac{d \Gamma^{D^{(*)}}}{d \theta} d \theta-\int_{\pi / 2}^{\pi} \frac{d \Gamma^{D^{(*)}}}{d \theta} d \theta}{\int_{0}^{\pi / 2} \frac{d \Gamma^{D^{(*)}}}{d \theta} d \theta+\int_{\pi / 2}^{\pi} \frac{d \Gamma^{D^{(*)}}}{d \theta} d \theta} \\
& =\frac{\int b_{\tau}^{D^{(*)}}\left(q^{2}\right) d q^{2}}{\Gamma^{D^{(*)}}}
\end{aligned}
$$

where $\Gamma^{D^{(*)}}$ is the total decay width of $D^{(*)}$ and the angle $\theta$ has already been defined above. Note that, while the branching fractions depend on the functions $a_{\ell}^{D^{(*)}}$ and $c_{\ell}^{D^{(*)}}$, the forward-backward asymmetry depends only on $b_{\ell}^{D^{(*)}}$. Hence, they provide complementary information on the nature of the short distance physics.

\section{$4 \quad \bar{B} \rightarrow D$ form factors}

The hadronic matrix elements for $\bar{B} \rightarrow D$ transition are parametrised by ${ }^{2}$

$$
\begin{aligned}
\left\langle D\left(p_{D}, M_{D}\right)\left|\bar{c} \gamma^{\mu} b\right| \bar{B}\left(p_{B}, M_{B}\right)\right\rangle= & F_{+}\left(q^{2}\right)\left[\left(p_{B}+p_{D}\right)^{\mu}-\frac{M_{B}^{2}-M_{D}^{2}}{q^{2}} q^{\mu}\right] \\
& +F_{0}\left(q^{2}\right) \frac{M_{B}^{2}-M_{D}^{2}}{q^{2}} q^{\mu} \\
\left\langle D\left(p_{D}, M_{D}\right)\left|\bar{c} \gamma^{\mu} \gamma_{5} b\right| \bar{B}\left(p_{B}, M_{B}\right)\right\rangle= & 0 \\
\left\langle D\left(p_{D}, M_{D}\right)|\bar{c} b| \bar{B}\left(p_{B}, M_{B}\right)\right\rangle & =F_{0}\left(q^{2}\right) \frac{M_{B}^{2}-M_{D}^{2}}{m_{b}-m_{c}} \\
\left\langle D\left(p_{D}, M_{D}\right)\left|\bar{c} \gamma_{5} b\right| \bar{B}\left(p_{B}, M_{B}\right)\right\rangle & =0 \\
\left\langle D\left(p_{D}, M_{D}\right)\left|\bar{c} \sigma^{\mu \nu} b\right| \bar{B}\left(p_{B}, M_{B}\right)\right\rangle & =-i\left(p_{B}^{\mu} p_{D}^{\nu}-p_{B}^{\nu} p_{D}^{\mu}\right) \frac{2 F_{T}\left(q^{2}\right)}{M_{B}+M_{D}} \\
\left\langle D\left(p_{D}, M_{D}\right)\left|\bar{c} \sigma^{\mu \nu} \gamma_{5} b\right| \bar{B}\left(p_{B}, M_{B}\right)\right\rangle & =\varepsilon^{\mu \nu \rho \sigma} p_{B \rho} p_{D \sigma} \frac{2 F_{T}\left(q^{2}\right)}{M_{B}+M_{D}}
\end{aligned}
$$

\footnotetext{
${ }^{2}$ We use the convention $\epsilon^{0123}=1$. This implies $\epsilon_{0123}=-1$.
} 
Note that eq. (4.3) and eq. (4.6) are not independent equations and follow from eq. (4.1) and eq. (4.5) respectively. Multiplying the left hand side of eq. (4.1) by $q_{\mu}$ one gets

$$
\begin{aligned}
q_{\mu}\left\langle D\left(p_{D}, m_{D}\right)\left|\bar{c} \gamma^{\mu} b\right| \bar{B}\left(p_{B}, M_{B}\right)\right\rangle & =\text { Inverse Fourier transform of }\left\langle D\left|i \partial_{\mu}\left(\bar{c} \gamma^{\mu} b\right)\right| B\right\rangle \\
& =\text { Inverse Fourier transform of }\left\langle D\left|\left(i \partial_{\mu} \bar{c} \gamma^{\mu} b+\bar{c} \gamma^{\mu} i \partial_{\mu} b\right)\right| B\right\rangle \\
& =\left(m_{b}-m_{c}\right)\left\langle D\left(p_{D}, M_{D}\right)|\bar{c} b| \bar{B}\left(p_{B}, M_{B}\right)\right\rangle
\end{aligned}
$$

Similarly, the term proportional to $F_{+}$in the right hand side of eq. (4.1) vanishes upon multiplication by $q_{\mu}$ and gives

$$
\text { r.h.s. }=F_{0}\left(q^{2}\right)\left(M_{B}^{2}-M_{D}^{2}\right) \text {. }
$$

Thus, eq. (4.7) and eq. (4.8) taken together give us eq. (4.3).

In order to get eq. (4.6) from eq. (4.5) one has to use the identity,

$$
\sigma^{\mu \nu} \gamma_{5}=\frac{i}{2} \varepsilon^{\mu \nu \alpha \beta} \sigma_{\alpha \beta}
$$

Substituting the above identity into the left hand side of eq. (4.6) one gets,

$$
\begin{aligned}
\left\langle D\left(p_{D}, M_{D}\right)\left|\bar{c} \sigma^{\mu \nu} \gamma_{5} b\right| \bar{B}\left(p_{B}, M_{B}\right)\right\rangle & =\frac{i}{2} \varepsilon^{\mu \nu \alpha \beta}\left\langle D\left(p_{D}, M_{D}\right)\left|\bar{c} \sigma_{\alpha \beta} b\right| \bar{B}\left(p_{B}, M_{B}\right)\right\rangle \\
& =\frac{i}{2} \varepsilon^{\mu \nu \alpha \beta}\left(-i\left(p_{B \alpha} p_{D \beta}-p_{B \beta} p_{D \alpha}\right) \frac{2 F_{T}\left(q^{2}\right)}{M_{B}+M_{D}}\right) \\
& =\varepsilon^{\mu \nu \alpha \beta} p_{B \alpha} p_{D \beta} \frac{2 F_{T}\left(q^{2}\right)}{M_{B}+M_{D}}
\end{aligned}
$$

The form factors $F_{0}\left(q^{2}\right)$ and $F_{+}\left(q^{2}\right)$ have been calculated using lattice QCD techniques in [19]..$^{3}$ They are given by the following expressions,

$$
\begin{aligned}
& F_{+}(z)=\frac{1}{\phi_{+}(z)} \sum_{k=0}^{3} a_{k}^{+} z^{k}, \\
& F_{0}(z)=\frac{1}{\phi_{0}(z)} \sum_{k=0}^{3} a_{k}^{0} z^{k}
\end{aligned}
$$

where

$$
z \equiv z\left(q^{2}\right)=\frac{\sqrt{\left(M_{B}+M_{D}\right)^{2}-q^{2}}-\sqrt{4 M_{B} M_{D}}}{\sqrt{\left(M_{B}+M_{D}\right)^{2}-q^{2}}+\sqrt{4 M_{B} M_{D}}} .
$$

\footnotetext{
${ }^{3}$ There has been another Lattice calculation of these form factors with similar results [22].
} 

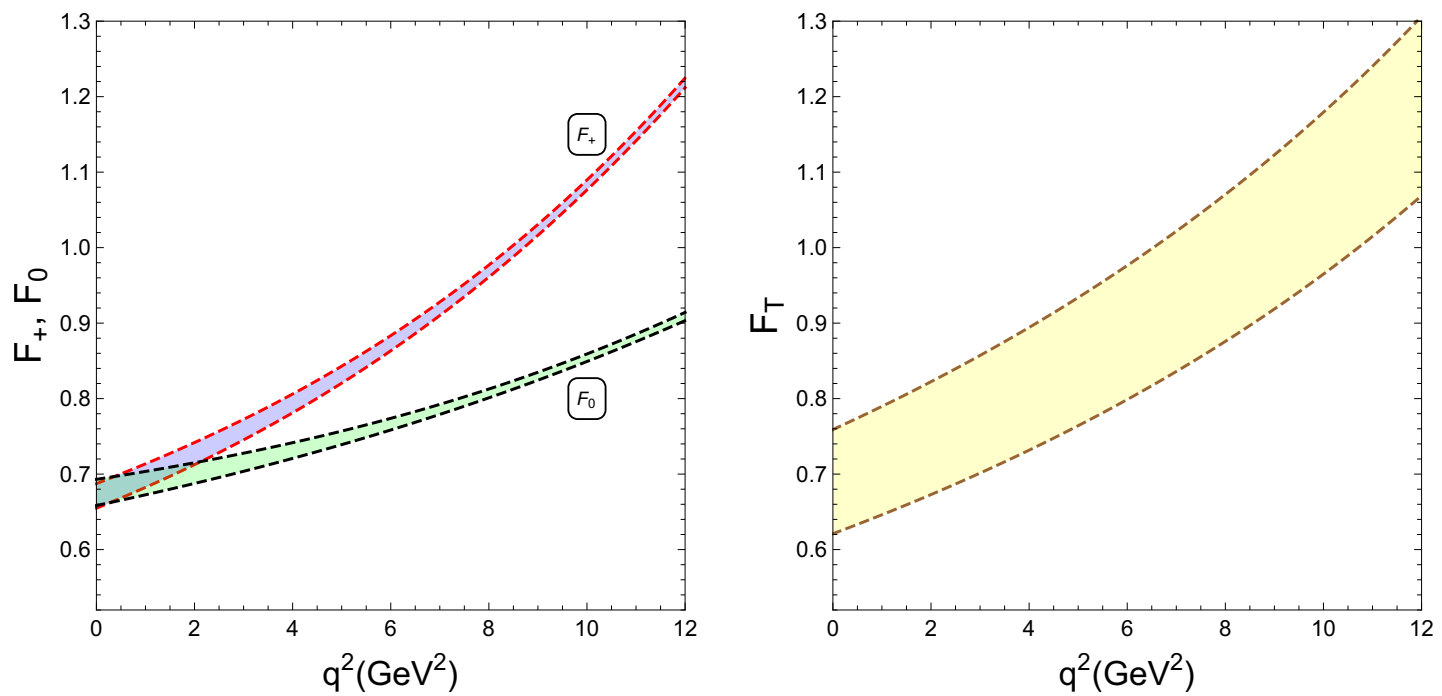

Figure 1. The $q^{2}$ dependence of the form factors $F_{0}, F_{+}$and $F_{T}$. The uncertainty bands for $F_{0}$ and $F_{+}$correspond to a $\chi^{2} \leq 1.646$ where the $\chi^{2}$ is computed using the expression $\chi^{2}(\mathbf{x})=\left(\mathbf{x}-\mathbf{x}_{\mathbf{0}}\right)^{T} \mathbf{V}^{-1}\left(\mathbf{x}-\mathbf{x}_{\mathbf{0}}\right)$ where $\mathbf{x}=\left(a_{0}^{+}, a_{1}^{+}, a_{2}^{+}, a_{3}^{+}, a_{0}^{0}, a_{1}^{0}, a_{2}^{0}, a_{3}^{0}\right)$ and $x_{0}$ consists of the central values given in table 2 . The covariance matrix $V$ is computed from the correlation matrix $\rho_{i j}$ given in table 3 using the formula $V_{i j}=\sigma_{i}(\mathbf{x}) \rho_{i j} \sigma_{j}(\mathbf{x})$ where $\sigma(\mathbf{x})$ is the vector of uncertainties given in tables 2 . The uncertainty band for $F_{T}$ is obtained by simply taking a $\pm 10 \%$ uncertainty on the central value.

The functions $\phi_{+}(z)$ and $\phi_{0}(z)$ are given by,

$$
\begin{aligned}
\phi_{+}(z) & =1.1213 \frac{(1+z)^{2}(1-z)^{1 / 2}}{[(1+r)(1-z)+2 \sqrt{r}(1+z)]^{5}}, \\
\phi_{0}(z) & =0.5299 \frac{(1+z)(1-z)^{3 / 2}}{[(1+r)(1-z)+2 \sqrt{r}(1+z)]^{4}},
\end{aligned}
$$

where, $r=M_{D} / M_{B}$.

The central values, uncertainties, and correlation matrix for the parameters $a_{k}^{0}$ and $a_{k}^{+}$ are shown in tables 2 and 3 .

As the tensor form factor $F_{T}$ has not been computed from lattice QCD, we have taken them from [65]. Following [65], we write $F_{T}\left(q^{2}\right)$ as,

$$
F_{T}\left(q^{2}\right)=\frac{0.69}{\left(1-\frac{q^{2}}{(6.4 \mathrm{GeV})^{2}}\right)\left(1-0.56 \frac{q^{2}}{(6.4 \mathrm{GeV})^{2}}\right)}
$$

In figure 1 , we show the $q^{2}$ dependences of $F_{0}, F_{+}$and $F_{T}$ following the above expressions. 


\begin{tabular}{|l|cccc|cccc|}
\hline & $a_{0}^{+}$ & $a_{1}^{+}$ & $a_{2}^{+}$ & $a_{3}^{+}$ & $a_{0}^{0}$ & $a_{1}^{0}$ & $a_{2}^{0}$ & $a_{3}^{0}$ \\
\hline Values & 0.01261 & -0.0963 & 0.37 & -0.05 & 0.01140 & -0.0590 & 0.19 & -0.03 \\
Uncertainties & 0.00010 & 0.0033 & 0.11 & 0.90 & 0.00009 & 0.0028 & 0.10 & 0.87 \\
\hline
\end{tabular}

Table 2. The central values and uncertainties for the parameters $a_{k}^{0}$ and $a_{k}^{+}$from ref. [19] (table XI of their arXiv version 1).

\begin{tabular}{|r|rrrrrrrrr|}
\hline & $a_{0}^{+}$ & $a_{1}^{+}$ & $a_{2}^{+}$ & $a_{3}^{+}$ & $a_{0}^{0}$ & $a_{1}^{0}$ & $a_{2}^{0}$ & $a_{3}^{0}$ \\
\hline$a_{0}^{+}$ & 1.00000 & 0.24419 & -0.08658 & 0.01207 & 0.00000 & 0.23370 & 0.03838 & -0.05639 \\
$a_{1}^{+}$ & & 1.00000 & -0.57339 & 0.25749 & 0.00000 & 0.80558 & -0.25493 & -0.15014 \\
$a_{2}^{+}$ & & & 1.00000 & -0.64492 & 0.00000 & -0.44966 & 0.66213 & 0.05120 \\
$a_{3}^{+}$ & & & & 1.00000 & 0.00000 & 0.11311 & -0.20100 & 0.23714 \\
$a_{0}^{0}$ & & & & & 1.00000 & 0.00000 & 0.00000 & 0.00000 \\
$a_{1}^{0}$ & & & & & & 1.00000 & -0.44352 & 0.02485 \\
$a_{2}^{0}$ & & & & & & & 1.00000 & -0.46248 \\
$a_{3}^{0}$ & & & & & & & & 1.00000 \\
\hline
\end{tabular}

Table 3. The correlation matrix for the parameters $a_{k}^{0}$ and $a_{k}^{+}$from ref. [19] (table XI of their arXiv version 1).

\section{$5 \quad \bar{B} \rightarrow D^{*}$ form factors}

The hadronic matrix elements for $\bar{B} \rightarrow D^{*}$ transition are parametrised by

$$
\begin{aligned}
& \left\langle D^{*}\left(p_{D^{*}}, M_{D^{*}}\right)\left|\bar{c} \gamma_{\mu} b\right| \bar{B}\left(p_{B}, M_{B}\right)\right\rangle=i \varepsilon_{\mu \nu \rho \sigma} \epsilon^{\nu *} p_{B}^{\rho} p_{D^{*}}^{\sigma} \frac{2 V\left(q^{2}\right)}{M_{B}+M_{D^{*}}} \\
& \left\langle D^{*}\left(p_{D^{*}, M_{D^{*}}}\right)\left|\bar{c} \gamma_{\mu} \gamma_{5} b\right| \bar{B}\left(p_{B}, M_{B}\right)\right\rangle=2 M_{D^{*}} \frac{\epsilon^{*} \cdot q}{q^{2}} q_{\mu} A_{0}\left(q^{2}\right)+\left(M_{B}+M_{D^{*}}\right)\left[\epsilon_{\mu}^{*}-\frac{\epsilon^{*} \cdot q}{q^{2}} q_{\mu}\right] A_{1}\left(q^{2}\right) \\
& -\frac{\epsilon^{*} \cdot q}{M_{B}+M_{D^{*}}}\left[\left(p_{B}+p_{D^{*}}\right)_{\mu}-\frac{M_{B}^{2}-M_{D^{*}}^{2}}{q^{2}} q_{\mu}\right] A_{2}\left(q^{2}\right) \\
& \left\langle D^{*}\left(p_{D^{*}}, M_{D^{*}}\right)|\bar{c} b| \bar{B}\left(p_{B}, M_{B}\right)\right\rangle=0 \\
& \left\langle D^{*}\left(p_{D^{*}}, M_{D^{*}}\right)\left|\bar{c} \gamma_{5} b\right| \bar{B}\left(p_{B}, M_{B}\right)\right\rangle=-\epsilon^{*} . q \frac{2 M_{D^{*}}}{m_{b}+m_{c}} A_{0}\left(q^{2}\right) \\
& \left\langle D^{*}\left(p_{D^{*}}, M_{D^{*}}\right)\left|\bar{c} \sigma_{\mu \nu} b\right| \bar{B}\left(p_{B}, M_{B}\right)\right\rangle=-\varepsilon_{\mu \nu \alpha \beta}\left[-\epsilon^{\alpha *}\left(p_{D^{*}}+p_{B}\right)^{\beta} T_{1}\left(q^{2}\right)\right. \\
& +\frac{M_{B}^{2}-M_{D^{*}}^{2}}{q^{2}} \epsilon^{* \alpha} q^{\beta}\left(T_{1}\left(q^{2}\right)-T_{2}\left(q^{2}\right)\right) \\
& \left.+2 \frac{\epsilon^{*} \cdot q}{q^{2}} p_{B}^{\alpha} p_{D^{*}}^{\beta}\left(T_{1}\left(q^{2}\right)-T_{2}\left(q^{2}\right)-\frac{q^{2}}{M_{B}^{2}-M_{D^{*}}^{2}} T_{3}\left(q^{2}\right)\right)\right]
\end{aligned}
$$

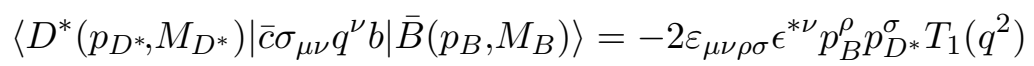


None of the form factors $V, A_{0}, A_{1}, A_{2}, T_{1}, T_{2}, T_{3}$ has been calculated in Lattice QCD. We used the heavy quark effective theory (HQET) form factors based on [66]. These form factors can be written in terms of the HQET form factors in the following way $[36,66]$,

$$
\begin{aligned}
& V\left(q^{2}\right)=\frac{M_{B}+M_{D^{*}}}{2 \sqrt{M_{B} M_{D^{*}}}} h_{V}\left(w\left(q^{2}\right)\right), \\
& A_{1}\left(q^{2}\right)=\frac{\left(M_{B}+M_{D^{*}}\right)^{2}-q^{2}}{2 \sqrt{M_{B} M_{D^{*}}}\left(M_{B}+M_{D^{*}}\right)} h_{A_{1}}\left(w\left(q^{2}\right)\right) \\
& A_{2}\left(q^{2}\right)=\frac{M_{B}+M_{D^{*}}}{2 \sqrt{M_{B} M_{D^{*}}}}\left[h_{A_{3}}\left(w\left(q^{2}\right)\right)+\frac{M_{D^{*}}}{M_{B}} h_{A_{2}}\left(w\left(q^{2}\right)\right)\right] \\
& A_{0}\left(q^{2}\right)=\frac{1}{2 \sqrt{M_{B} M_{D^{*}}}}\left[\frac{\left(M_{B}+M_{D^{*}}\right)^{2}-q^{2}}{2 M_{D^{*}}} h_{A_{1}}\left(w\left(q^{2}\right)\right)\right. \\
& \left.-\frac{M_{B}^{2}-M_{D^{*}}^{2}+q^{2}}{2 M_{B}} h_{A_{2}}\left(w\left(q^{2}\right)\right)-\frac{M_{B}^{2}-M_{D^{*}}^{2}-q^{2}}{2 M_{D^{*}}} h_{A_{3}}\left(w\left(q^{2}\right)\right)\right] \\
& T_{1}\left(q^{2}\right)=\frac{1}{2 \sqrt{M_{B} M_{D^{*}}}}\left[\left(M_{B}+M_{D^{*}}\right) h_{T_{1}}\left(w\left(q^{2}\right)\right)-\left(M_{B}-M_{D^{*}}\right) h_{T_{2}}\left(w\left(q^{2}\right)\right)\right] \\
& T_{2}\left(q^{2}\right)=\frac{1}{2 \sqrt{M_{B} M_{D^{*}}}}\left[\frac{\left(M_{B}+M_{D^{*}}\right)^{2}-q^{2}}{M_{B}+M_{D^{*}}} h_{T_{1}}\left(w\left(q^{2}\right)\right)\right. \\
& \left.-\frac{\left(M_{B}-M_{D^{*}}\right)^{2}-q^{2}}{M_{B}-M_{D^{*}}} h_{T_{2}}\left(w\left(q^{2}\right)\right)\right] \\
& T_{3}\left(q^{2}\right)=\frac{1}{2 \sqrt{M_{B} M_{D^{*}}}}\left[\left(M_{B}-M_{D^{*}}\right) h_{T_{1}}\left(w\left(q^{2}\right)\right)-\left(M_{B}+M_{D^{*}}\right) h_{T_{2}}\left(w\left(q^{2}\right)\right)\right. \\
& \left.-2 \frac{M_{B}^{2}-M_{D^{*}}^{2}}{M_{B}} h_{T_{3}}\left(w\left(q^{2}\right)\right)\right],
\end{aligned}
$$

where,

$$
\begin{aligned}
h_{V}(w)= & R_{1}(w) h_{A_{1}}(w) \\
h_{A_{2}}(w)= & \frac{R_{2}(w)-R_{3}(w)}{2 r_{D^{*}}} h_{A_{1}}(w) \\
h_{A_{3}}(w)= & \frac{R_{2}(w)+R_{3}(w)}{2} h_{A_{1}}(w) \\
h_{T_{1}}(w)= & \frac{1}{2\left(1+r_{D^{*}}^{2}-2 r_{D^{*}} w\right)}\left[\frac{m_{b}-m_{c}}{M_{B}-M_{D^{*}}}\left(1-r_{D^{*}}\right)^{2}(w+1) h_{A_{1}}(w)\right. \\
& \left.-\frac{m_{b}+m_{c}}{M_{B}+M_{D^{*}}}\left(1+r_{D^{*}}\right)^{2}(w-1) h_{V}(w)\right] \\
h_{T_{2}}(w)= & \frac{\left(1-r_{D^{*}}^{2}\right)(w+1)}{2\left(1+r_{D^{*}}^{2}-2 r_{D^{*}} w\right)}\left[\frac{m_{b}-m_{c}}{M_{B}-M_{D^{*}}} h_{A_{1}}(w)-\frac{m_{b}+m_{c}}{M_{B}+M_{D^{*}}} h_{V}(w)\right] \\
h_{T_{3}}(w)= & -\frac{1}{2\left(1+r_{D^{*}}\right)\left(1+r_{D^{*}}^{2}-2 r_{D^{*}} w\right)}\left[2 \frac{m_{b}-m_{c}}{M_{B}-M_{D^{*}}} r_{D^{*}}(w+1) h_{A_{1}}(w)\right. \\
& -\frac{m_{b}-m_{c}}{M_{B}-M_{D^{*}}}\left(1+r_{D^{*}}^{2}-2 r_{D^{*}} w\right)\left(h_{A_{3}}(w)-r_{D^{*}} h_{A_{2}}(w)\right) \\
& \left.-\frac{m_{b}+m_{c}}{M_{B}+M_{D^{*}}}\left(1+r_{D^{*}}\right)^{2} h_{V}(w)\right]
\end{aligned}
$$



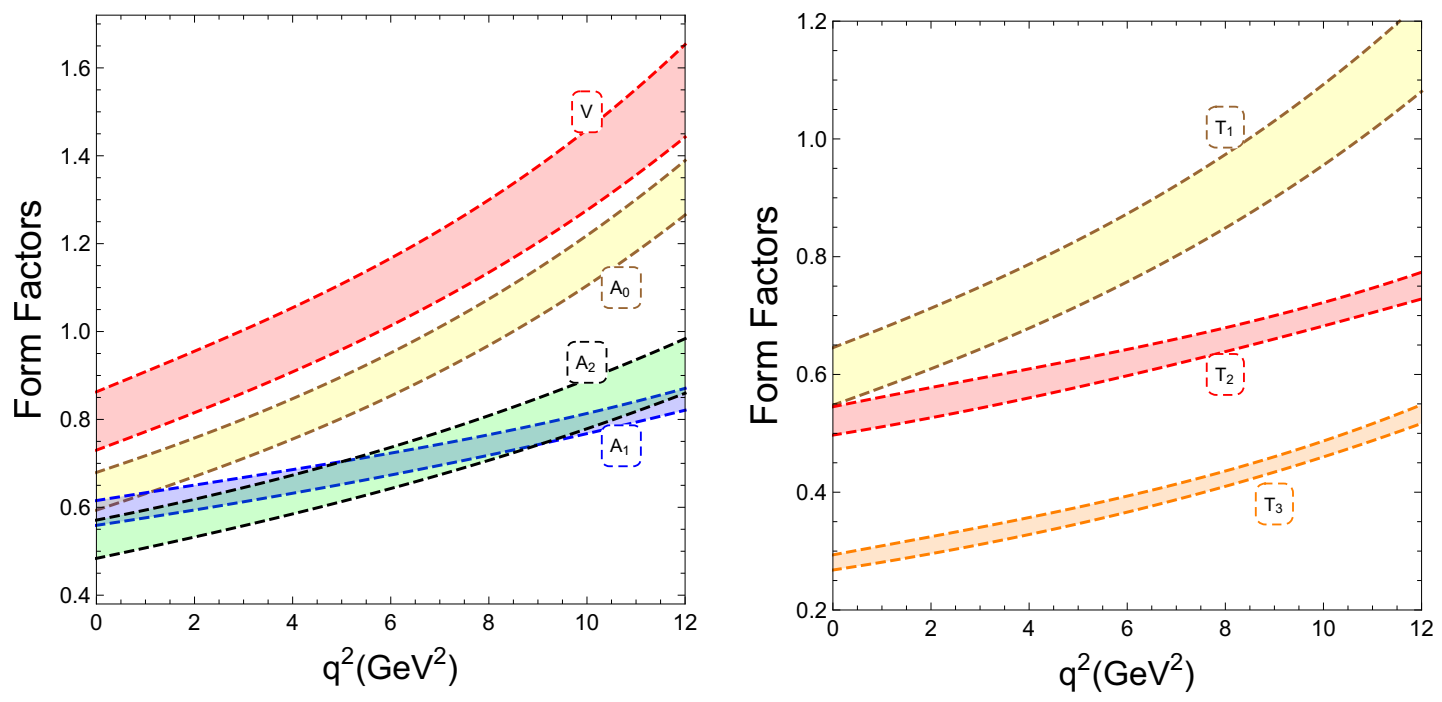

Figure 2. The $q^{2}$ dependence of the $B \rightarrow D^{*}$ form factors. The bands correspond to two times the uncertainties given in eq. (5.11).

$$
\begin{aligned}
h_{A_{1}}(w) & =h_{A_{1}}(1)\left[1-8 \rho_{D^{*}}^{2} z+\left(53 \rho_{D^{*}}^{2}-15\right) z^{2}-\left(231 \rho_{D^{*}}^{2}-91\right) z^{3}\right] \\
R_{1}(w) & =R_{1}(1)-0.12(w-1)+0.05(w-1)^{2} \\
R_{2}(w) & =R_{2}(1)+0.11(w-1)-0.06(w-1)^{2} \\
R_{3}(w) & =1.22-0.052(w-1)+0.026(w-1)^{2}
\end{aligned}
$$

Here, $r_{D^{*}}=M_{D^{*}} / M_{B}, w\left(q^{2}\right)=\left(M_{B}^{2}+M_{D^{*}}^{2}-q^{2}\right) / 2 M_{B} M_{D^{*}}$ and $z(w)=(\sqrt{w+1}-$ $\sqrt{2}) /(\sqrt{w+1}+\sqrt{2})$.

The numerical values of the relevant parameters of the form factors along with their respective $1 \sigma$ errors are given by

$$
\begin{aligned}
R_{1}(1) & =1.406 \pm 0.033, R_{2}(1)=0.853 \pm 0.020, \rho_{D^{*}}^{2}=1.207 \pm 0.026[17] \\
h_{A_{1}}(1) & =0.906 \pm 0.013[67] .
\end{aligned}
$$

In figure 2 we show the $q^{2}$ dependence of the form factors using these numerical values. As there have been no lattice calculations of these form factors, in order to be conservative, we use two times larger uncertainties than those quoted above.

\section{Expressions for $a_{\ell}^{D}, b_{\ell}^{D}$ and $c_{\ell}^{D}$ for $\bar{B} \rightarrow D \ell \bar{\nu}_{\ell}$}

The quantities $a_{\ell}^{D}, b_{\ell}^{D}$ and $c_{\ell}^{D}$ for positive helicity lepton are given by:

$$
\begin{aligned}
a_{\ell}^{D}(+)= & \frac{2\left(M_{B}^{2}-M_{D}^{2}\right)^{2}}{\left(m_{b}-m_{c}\right)^{2}}\left|\mathbf{C}_{\mathbf{S L}}^{\ell}\right|^{\mathbf{2}} \mathbf{F}_{\mathbf{0}}^{\mathbf{2}} \\
& +m_{\ell}\left[\frac{4\left(M_{B}^{2}-M_{D}^{2}\right)^{2}}{q^{2}\left(m_{b}-m_{c}\right)} \mathcal{R}\left(\mathbf{C}_{\mathbf{V L}}^{\ell} \mathbf{C}_{\mathbf{S L}}^{\ell *}\right) \mathbf{F}_{\mathbf{0}}^{\mathbf{2}}\right] \\
& +m_{\ell}^{2}\left[\frac{2\left(M_{B}^{2}-M_{D}^{2}\right)^{2}}{q^{4}}\left|\mathbf{C}_{\mathbf{V L}}^{\ell}\right|^{\mathbf{2}} \mathbf{F}_{\mathbf{0}}^{\mathbf{2}}\right]
\end{aligned}
$$




$$
\begin{aligned}
b_{\ell}^{D}(+)= & -m_{\ell}\left[\frac{8\left|p_{D}\right| M_{B}\left(M_{B}^{2}-M_{D}^{2}\right)}{q^{2}\left(m_{b}-m_{c}\right)} \mathcal{R}\left(\mathbf{C}_{\mathbf{S L}}^{\ell} \mathbf{C}_{\mathbf{V L}}^{\ell *}\right) \mathbf{F}_{\mathbf{0}} \mathbf{F}_{+}\right] \\
& -m_{\ell}^{2}\left[\frac{8\left|p_{D}\right| M_{B}\left(M_{B}^{2}-M_{D}^{2}\right)}{q^{4}}\left|\mathbf{C}_{\mathbf{V L}}^{\ell}\right|^{2} \mathbf{F}_{\mathbf{0}} \mathbf{F}_{+}\right] \\
c_{\ell}^{D}(+)= & m_{\ell}^{2}\left[\frac{8\left|p_{D}\right|^{2} M_{B}^{2}}{q^{4}}\left|\mathbf{C}_{\mathbf{V L}}^{\ell}\right|^{2} \mathbf{F}_{+}^{2}\right]
\end{aligned}
$$

Their expressions for the negative helicity lepton are,

$$
\begin{aligned}
& a_{\ell}^{D}(-)=\frac{8 M_{B}^{2}\left|p_{D}\right|^{2}}{q^{2}}\left|\mathbf{C}_{\mathbf{V L}}^{\ell}\right|^{\mathbf{2}} \mathbf{F}_{+}^{2} \\
& b_{\ell}^{D}(-)=0 \\
& c_{\ell}^{D}(-)=-\frac{8 M_{B}^{2}\left|p_{D}\right|^{2}}{q^{2}}\left|\mathbf{C}_{\mathbf{V L}}^{\ell}\right|^{2} \mathbf{F}_{+}^{2}
\end{aligned}
$$

Note that, the WCs $C_{A L}^{\ell}$ and $C_{P L}^{\ell}$ do not contribute to this decay. This is because the corresponding QCD matrix elements vanish, as can be seen from eqs. (4.2) and (4.4).

The lepton mass dependence of the various terms can also be understood easily. As the vector operators do not change the chirality of the fermion line, because of the left chiral nature of the neutrino, the outgoing (negatively charged) lepton also has negative chirality (and hence negative helicity in the massless limit). Thus the production of a left-handed lepton through the vector operator does not need a mass insertion. By a similar argument, one can see that the production of a right-handed lepton through the scalar operator does not need any mass insertion. The amplitude for the production of a right-handed lepton through a vector operator, on the other hand, clearly requires a mass insertion in order to flip the lepton helicity. This explains why the terms proportional to $\left|C_{V L}^{\ell}\right|^{2}$ in eqs. (6.1)-(6.3) have $m_{\ell}^{2}$ and the interference terms proportional to $\mathcal{R}\left(C_{\mathrm{SL}}^{\ell} C_{\mathrm{VL}}^{\ell *}\right)$ have $m_{\ell}$ in front, while there is no such dependence in eqs. (6.4)-(6.6).

The full expressions for $a_{\ell}^{D}, b_{\ell}^{D}$ and $c_{\ell}^{D}$ including all the operators in eq. (2.6) are shown in appendix A.

\section{Expressions for $a_{\ell}^{D^{*}}, b_{\ell}^{D^{*}}$ and $c_{\ell}^{D^{*}}$ for $\bar{B} \rightarrow D^{*} \ell \bar{\nu}_{\ell}$}

The quantities $a_{\ell}^{D^{*}}, b_{\ell}^{D^{*}}$ and $c_{\ell}^{D^{*}}$ for positive and negative helicitiy leptons are given by,

$$
\begin{aligned}
a_{\ell}^{D^{*}}(-)= & \frac{8 M_{B}^{2}\left|p_{D^{*}}\right|^{2}}{\left(M_{B}+M_{D^{*}}\right)^{2}}\left|\mathbf{C}_{\mathbf{V L}}^{\ell}\right|^{\mathbf{2}} \mathbf{V}^{\mathbf{2}}+\frac{\left(M_{B}+M_{D^{*}}\right)^{2}\left(8 M_{D^{*}}^{2} q^{2}+\lambda\right)}{2 M_{D^{*}}^{2} q^{2}}\left|\mathbf{C}_{\mathbf{A L}}^{\ell}\right|^{\mathbf{2}} \mathbf{A}_{\mathbf{1}}^{\mathbf{2}} \\
& +\frac{8 M_{B}^{4}\left|p_{D^{*}}\right|^{4}}{M_{D^{*}}^{2}\left(M_{B}+M_{D^{*}}\right)^{2} q^{2}}\left|\mathbf{C}_{\mathbf{A L}}^{\ell}\right|^{\mathbf{2}} \mathbf{A}_{\mathbf{2}}^{\mathbf{2}} \\
& -\frac{4\left|p_{D^{*}}\right|^{2} M_{B}^{2}\left(M_{B}^{2}-M_{D^{*}}^{2}-q^{2}\right)}{M_{D^{*}}^{2} q^{2}}\left|\mathbf{C}_{\mathbf{A L}}^{\ell}\right|^{\mathbf{2}}\left(\mathbf{A}_{\mathbf{1}} \mathbf{A}_{\mathbf{2}}\right)
\end{aligned}
$$




$$
\begin{aligned}
& b_{\ell}^{D^{*}}(-)=-16\left|p_{D^{*}}\right| M_{B} \mathcal{R}\left(\mathbf{C}_{\mathbf{V L}}^{\ell} \mathbf{C}_{\mathbf{A L}}^{\ell *}\right)\left(\mathbf{V A}_{\mathbf{1}}\right) \\
& c_{\ell}^{D^{*}}(-)=\frac{8\left|p_{D^{*}}\right|^{2} M_{B}^{2}}{\left(M_{B}+M_{D^{*}}\right)^{2}}\left|\mathbf{C}_{\mathbf{V L}}^{\ell}\right|^{2} \mathbf{V}^{\mathbf{2}}-\frac{\left(M_{B}+M_{D^{*}}\right)^{2} \lambda}{2 M_{D^{*}}^{2} q^{2}}\left|\mathbf{C}_{\mathbf{A L}}^{\ell}\right|^{2} \mathbf{A}_{\mathbf{1}}^{2} \\
& -\frac{8\left|p_{D^{*}}\right|^{4} M_{B}^{4}}{\left(M_{B}+M_{D^{*}}\right)^{2} M_{D^{*}}^{2} q^{2}}\left|\mathbf{C}_{\mathbf{A L}}^{\ell}\right|^{2} \mathbf{A}_{\mathbf{2}}^{2} \\
& +\frac{4\left|p_{D^{*}}\right|^{2} M_{B}^{2}\left(M_{B}^{2}-M_{D^{*}}^{2}-q^{2}\right)}{M_{D^{*}}^{2} q^{2}}\left|\mathbf{C}_{\mathbf{A L}}^{\ell}\right|^{2}\left(\mathbf{A}_{\mathbf{1}} \mathbf{A}_{\mathbf{2}}\right) \\
& a_{\ell}^{D^{*}}(+)=\frac{8\left|p_{D^{*}}\right|^{2} M_{B}^{2}}{\left(m_{b}+m_{c}\right)^{2}}\left|\mathbf{C}_{\mathbf{P L}}^{\ell}\right|^{2} \mathbf{A}_{\mathbf{0}}^{\mathbf{2}} \\
& -m_{\ell}\left[\frac{16\left|p_{D^{*}}\right|^{2} M_{B}^{2}}{\left(m_{b}+m_{c}\right) q^{2}} \mathcal{R}\left(\mathbf{C}_{\mathbf{A L}}^{\ell} \mathbf{C}_{\mathbf{P L}}^{\ell *}\right) \mathbf{A}_{\mathbf{0}}^{2}\right] \\
& +m_{\ell}^{2}\left[\frac{8\left|p_{D^{*}}\right|^{2} M_{B}^{2}}{q^{4}}\left|\mathbf{C}_{\mathbf{A L}}^{\ell}\right|^{2} \mathbf{A}_{\mathbf{0}}^{\mathbf{2}}+\frac{8\left|p_{D^{*}}\right|^{2} M_{B}^{2}}{\left(M_{B}+M_{D^{*}}\right)^{2} q^{2}}\left|\mathbf{C}_{\mathbf{V L}}^{\ell}\right|^{2} \mathbf{V}^{\mathbf{2}}\right. \\
& \left.+\frac{2\left(M_{B}+M_{D^{*}}\right)^{2}}{q^{2}}\left|\mathbf{C}_{\mathbf{A L}}^{\ell}\right|^{2} \mathbf{A}_{\mathbf{1}}^{2}\right] \\
& b_{\ell}^{D^{*}}(+)=m_{\ell}\left[\frac{4\left|p_{D^{*}}\right| M_{B}\left(M_{B}+M_{D^{*}}\right)\left(M_{B}^{2}-M_{D^{*}}^{2}-q^{2}\right)}{M_{D^{*}}\left(m_{b}+m_{c}\right) q^{2}} \mathcal{R}\left(\mathbf{C}_{\mathbf{A L}}^{\ell} \mathbf{C}_{\mathbf{P L}}^{\ell *}\right) \mathbf{A}_{\mathbf{0}} \mathbf{A}_{\mathbf{1}}\right. \\
& \left.-\frac{16}{\left(m_{b}+m_{c}\right)} \frac{\left|p_{D^{*}}\right|^{3} M_{B}^{3}}{\left(M_{B}+M_{D^{*}}\right) M_{D^{*}} q^{2}} \mathcal{R}\left(\mathbf{C}_{\mathbf{A L}}^{\ell} \mathbf{C}_{\mathbf{P L}}^{\ell *}\right) \mathbf{A}_{\mathbf{0}} \mathbf{A}_{\mathbf{2}}\right] \\
& +m_{\ell}^{2}\left[-\frac{4\left|p_{D^{*}}\right| M_{B}\left(M_{B}+M_{D^{*}}\right)}{M_{D^{*}} q^{4}}\left(M_{B}^{2}-M_{D^{*}}^{2}-q^{2}\right)\left|\mathbf{C}_{\mathbf{A L}}^{\ell}\right|^{2} \mathbf{A}_{\mathbf{0}} \mathbf{A}_{\mathbf{1}}\right. \\
& \left.+\frac{16\left|p_{D^{*}}\right|^{3} M_{B}^{3}}{\left(M_{B}+M_{D^{*}}\right) M_{D^{*} q^{4}}}\left|\mathbf{C}_{\mathbf{A L}}^{\ell}\right|^{2} \mathbf{A}_{\mathbf{0}} \mathbf{A}_{\mathbf{2}}\right] \\
& c_{\ell}^{D^{*}}(+)=m_{\ell}^{2}\left[-\frac{8\left|p_{D^{*}}\right|^{2} M_{B}^{2}}{\left(M_{B}+M_{D^{*}}\right)^{2} q^{2}}\left|\mathbf{C}_{\mathbf{V L}}^{\ell}\right|^{\mathbf{2}} \mathbf{V}^{\mathbf{2}}+\frac{\left(M_{B}+M_{D^{*}}\right)^{2} \lambda}{2 M_{D^{*}}^{2} q^{4}}\left|\mathbf{C}_{\mathbf{A L}}^{\ell}\right|^{2} \mathbf{A}_{1}^{2}\right. \\
& +\frac{8\left|p_{D^{*}}\right|^{4} M_{B}^{4}}{M_{D^{*}}^{2}\left(M_{B}+M_{D^{*}}\right)^{2} q^{4}}\left|\mathbf{C}_{\mathbf{A L}}^{\ell}\right|^{2} \mathbf{A}_{\mathbf{2}}^{2} \\
& \left.-\frac{4\left|p_{D^{*}}\right|^{2} M_{B}^{2}}{M_{D^{*}}^{2} q^{4}}\left(M_{B}^{2}-M_{D^{*}}^{2}-q^{2}\right)\left|\mathbf{C}_{\mathbf{A L}}^{\ell}\right|^{2}\left(\mathbf{A}_{\mathbf{1}} \mathbf{A}_{\mathbf{2}}\right)\right]
\end{aligned}
$$

The WC $C_{S L}^{\ell}$ does not contribute to this decay because the corresponding QCD matrix element vanishes as can be seen from eq. (5.3). The lepton mass dependence of the various terms can be understood in the same way as the $\bar{B} \rightarrow D \ell \bar{\nu}_{\ell}$ decay. Note also the absence of interference terms proportional to $\mathcal{R}\left(C_{V L}^{\ell} C_{P L}^{\ell *}\right)$ in the above expressions.

We provide the completely general result taking into account all the operators in eq. (2.6) in appendix B. 

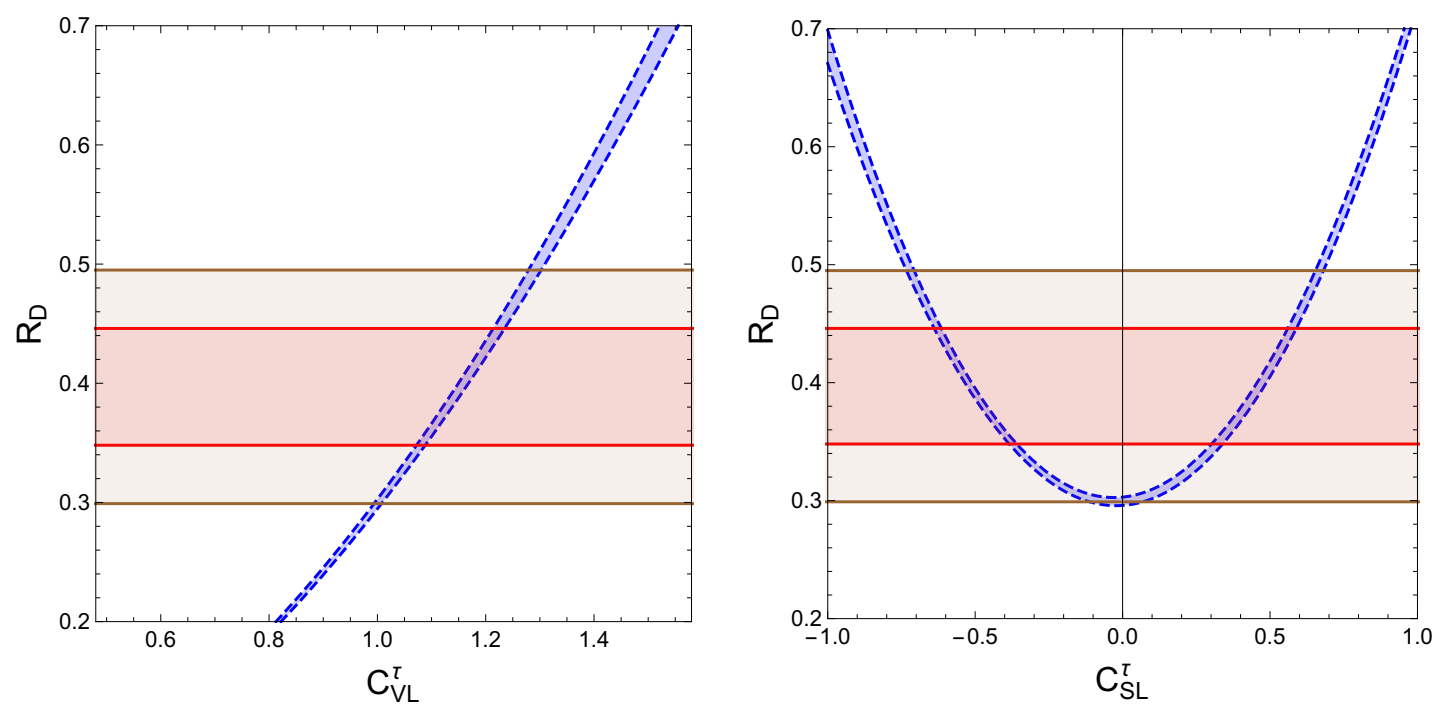

Figure 3. The dependence of $R_{D}$ with respect to the variation of the WCs $C_{V L}^{\tau}$ (left) and $C_{S L}^{\tau}$ (right).

\section{Results}

\subsection{Explaining $R_{D}$ alone}

As mentioned in section 6 , the $\bar{B} \rightarrow D \tau \bar{\nu}_{\tau}$ amplitude depends only on the WCs $C_{V L}^{\tau}$ and $C_{S L}^{\tau}$. In figure 3 , we show $R_{D}$ as function of $C_{V L}^{\tau}$ and $C_{S L}^{\tau}$. In the right plot, we set $C_{V L}^{\tau}$ to its $\mathrm{SM}$ value $\left.C_{V L}^{\tau}\right|_{\mathrm{SM}}=1$ and vary $C_{S L}^{\tau}$, while in the left plot, we hold $C_{S L}^{\tau}$ fixed at its $\mathrm{SM}$ value $\left.C_{S L}^{\tau}\right|_{\mathrm{SM}}=0$ and change $C_{V L}^{\tau}$. The red and brown shades correspond to the experimentally allowed $1 \sigma$ and $2 \sigma$ ranges (see table 1 ), for which we have added the statistical and systematic uncertainties in quadrature.

The ranges of $C_{V L}^{\tau}$ and $C_{S L}^{\tau}$ that are consistent with $R_{D}$ at $1 \sigma$ are shown in the second row of table 4 . The ranges for $C_{S L}^{\tau}$ are slightly asymmetric about zero because of its interference with $C_{V L}^{\tau}$. In the rows 3,4 and 5-8, we also show the predictions for $P_{\tau}(D)$, $\mathcal{A}_{F B}^{D}$ and $R_{D}$ in four different bins for the allowed ranges of $C_{V L}^{\tau}$ and $C_{S L}^{\tau}$. Note that, $\mathcal{A}_{F B}^{D}$ and the polarisation fraction $P_{\tau}(D)$ are independent of $C_{V L}^{\tau}$ if $C_{S L}^{\tau}$ is set to zero. This is because, in this case the differential decay rate is proportional to $\left|C_{V L}^{\tau}\right|^{2}$ and hence, the dependence on $C_{V L}^{\tau}$ drops out in $P_{\tau}(D)$ and $\mathcal{A}_{F B}^{D}$. This is why the ranges for $P_{\tau}(D)$ and $\mathcal{A}_{F B}^{D}$ in the third and fourth columns are identical. The binwise $R_{D}$ values are also graphically represented in figure 4 . The left and the right panels correspond to the WCs $C_{V L}^{\tau}$ and $C_{S L}^{\tau}$ respectively. The SM predictions are shown in red. One can conclude from figure 4 that the binwise $R_{D}$ does not help distinguish the two WCs $C_{V L}^{\tau}$ and $C_{S L}^{\tau}$.

The predictions for $P_{\tau}(D), \mathcal{A}_{F B}^{D}$ are pictorially presented in the left and middle panel of figure 5. As mentioned earlier, in the absence of $C_{S L}^{\tau}, P_{\tau}(D)$ and $\mathcal{A}_{F B}^{D}$ are completely independent of $C_{V L}^{\tau}$. Hence, neither measurement can distinguish between $C_{V L}^{\tau}=1$ and other values of $C_{V L}^{\tau}$. However, the predictions are very different for $C_{S L}^{\tau}$. Therefore, a 


\begin{tabular}{|c|l|c|c|c|}
\hline \multirow{7}{*}{} & & $\mathrm{SM}$ & $\begin{array}{c}\mathbf{C}_{\mathbf{V L}} \\
\left(\mathbf{C}_{\mathbf{S L}}=0\right)\end{array}$ & $\begin{array}{c}\mathbf{C}_{\mathbf{S L}} \\
\left(\mathbf{C}_{\mathbf{V L}}=1\right)\end{array}$ \\
\cline { 2 - 5 } & $1 \sigma$ range of the WC & & {$[1.073,1.222]$} & $\begin{array}{c}{[-0.656,-0.342]} \\
{[0.296,0.596]}\end{array}$ \\
\cline { 2 - 5 }$R_{D}[\mathrm{bin}]$ & $P_{\tau}(D)$ & {$[0.313,0.336]$} & {$[0.313,0.336]$} & {$[0.408,0.556]$} \\
\hline \multirow{3}{*}{} & $\mathcal{A}_{F B}^{D}$ & {$[-0.361,-0.358]$} & {$[-0.361,-0.358]$} & {$[-0.168,-0.022]$} \\
& {$\left[m_{\tau}^{2}-5\right] \mathrm{GeV}^{2}$} & {$[0.154,0.158]$} & {$[0.178,0.236]$} & {$[0.161,0.181]$} \\
& {$[5-7] \mathrm{GeV}^{2}$} & {$[0.980,1.003]$} & {$[1.127,1.505]$} & {$[1.125,1.502]$} \\
& {$[7-9] \mathrm{GeV}^{2}$} & {$[1.776,1.823]$} & {$[2.049,2.741]$} & {$[2.294,3.669]$} \\
\hline
\end{tabular}

Table 4. The values of the WCs consistent with the $1 \sigma$ experimental range for $R_{D}$ are shown in the second row. The subsequent rows show the predictions for $P_{\tau}(D), \mathcal{A}_{F B}^{D}$ and $R_{D}$ in four $q^{2}$ bins for the WC ranges shown in the second row.
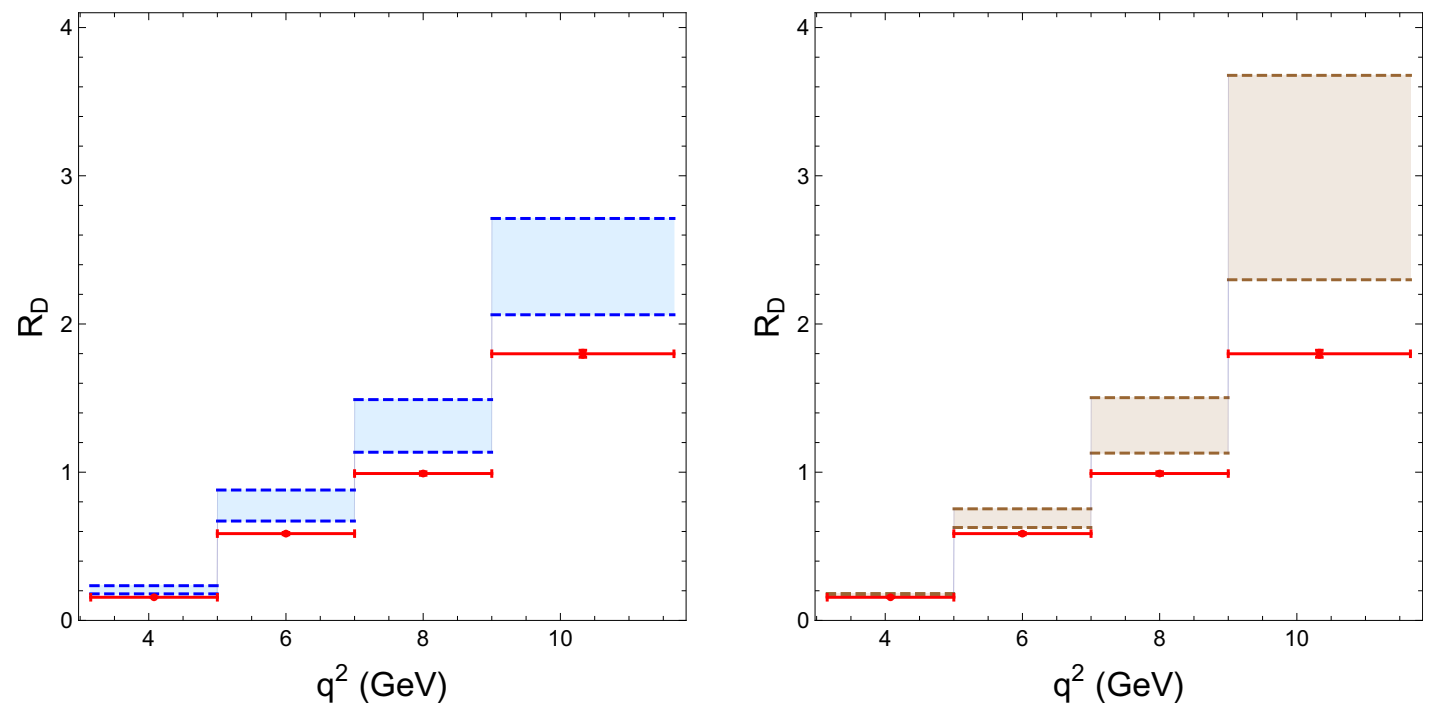

Figure 4. The binwise $R_{D}$ for four $q^{2}$ bins. On the left, $C_{V L}^{\tau}$ is varied, while on the right, $C_{S L}^{\tau}$ is varied within their $1 \sigma$ allowed ranges.

measurement of $P_{\tau}(D)$ will tell us whether NP in the form of scalar operator $\mathcal{O}_{S L}^{c b \tau}$ exists or not. Moreover, the two separate ranges of $C_{S L}^{\tau}$ which satisfy the experimental bounds give very different values of $\mathcal{A}_{F B}^{D}$, indicated by the subscripts "+" and "-" in the middle figure.

In the right panel of figure 5 , we also show the normalised differential decay width as a function of $q^{2}$. As for the case of $P_{\tau}(D)$ and $\mathcal{A}_{F B}^{D}$, the normalised differential decay width is independent of $C_{V L}^{\tau}$ for $C_{S L}^{\tau}=0$. The blue solid line is the SM prediction, and the black and red dashed lines are the predictions for two representative values of $C_{S L}^{\tau}, C_{S L}^{\tau}=-0.650$ and 0.310 respectively. While producing these plots, we have used the central values of the form 

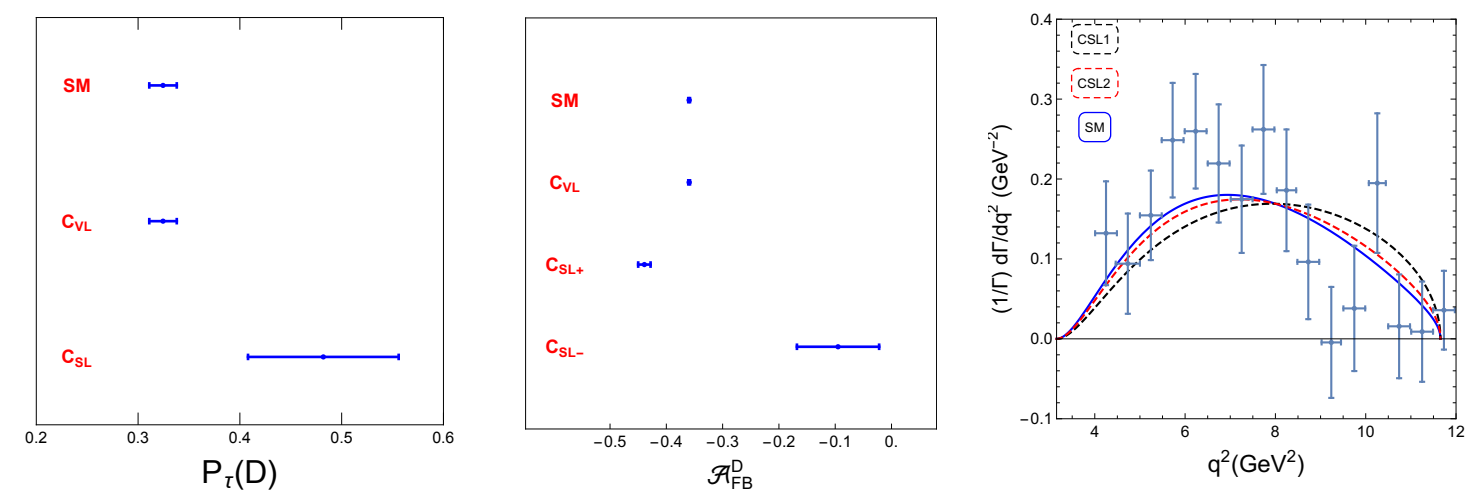

Figure 5. Predictions for the polarisation fraction $P_{\tau}(D)$ (left), $\mathcal{A}_{F B}^{D}$ (middle) and the differential decay width (right). The subscripts "+" and "" in the middle figure correspond to the two ranges of $C_{S L}^{\tau}$ that satisfy the experimental bounds of $R_{D}$. In the right graph showing the normalised differential decay width, the solid blue line is the SM prediction. The dashed black and red lines correspond to $C_{S L}^{\tau}=-0.650$ and 0.310 respectively. The data points shown on the right plot are due to the BaBar collaboration and are taken from [21].
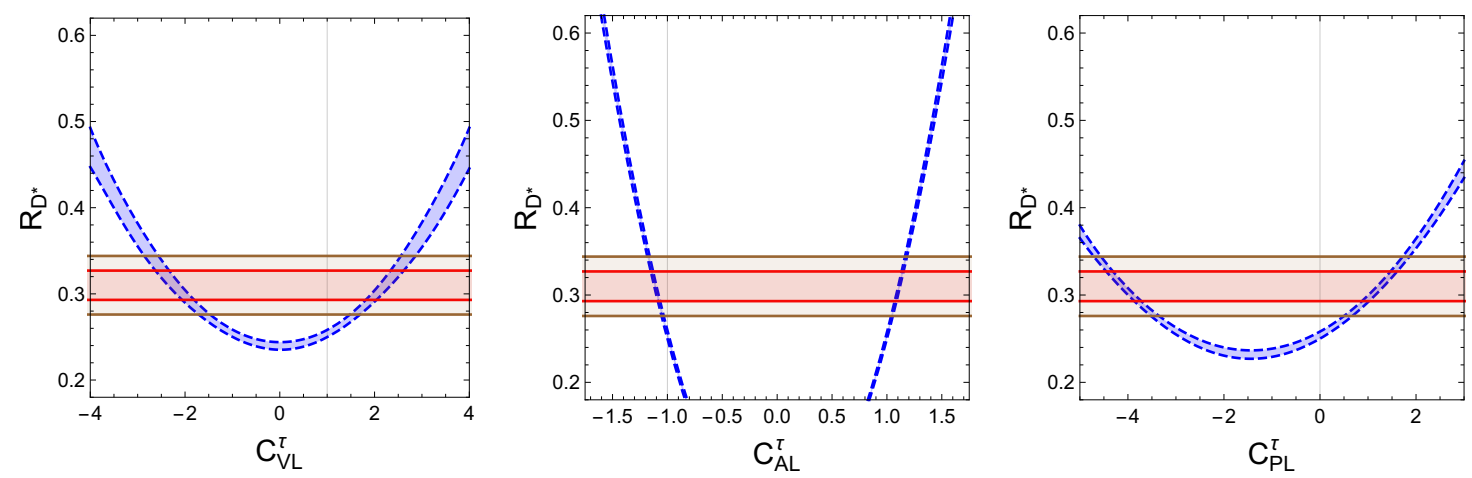

Figure 6. The dependence of $R_{D^{*}}$ with respect to the variation of the WCs $C_{V L}^{\tau}$ (left), $C_{A L}^{\tau}$ (middle) and $C_{P L}^{\tau}$ (right). A thin vertical line shows the SM values of the WCs.

factors. The blue data points are from the BaBar measurement reported in [21]. It is clear that the differential decay width is not a good discriminant of the various NP operators.

\subsection{Explaining $R_{D^{*}}$ alone}

The $\bar{B} \rightarrow D^{*} \tau \bar{\nu}_{\tau}$ decay amplitude depends on three WCs, $C_{V L}^{\tau}, C_{A L}^{\tau}$ and $C_{P L}^{\tau}$. In figure 6, we show $R_{D^{*}}$ as function of these WCs. In each of the plots, the WCs that are not varied are all set to their SM values. The red and brown shades correspond to the experimentally allowed $1 \sigma$ and $2 \sigma$ ranges respectively (see table 1 ).

The ranges of $C_{V L}^{\tau}, C_{A L}^{\tau}$ and $C_{P L}^{\tau}$ that are consistent with the experimental value of $R_{D^{*}}$ at $1 \sigma$ are shown in the second row of table 5 . We only show the ranges that are closest to the SM values of the WCs. In the rows 3,4 and 5-8, we also show the predictions for $P_{\tau}\left(D^{*}\right), \mathcal{A}_{F B}^{D^{*}}$ and $R_{D^{*}}$ in four different bins for these allowed ranges of $C_{V L}^{\tau}, C_{A L}^{\tau}$ and $C_{P L}^{\tau}$. 


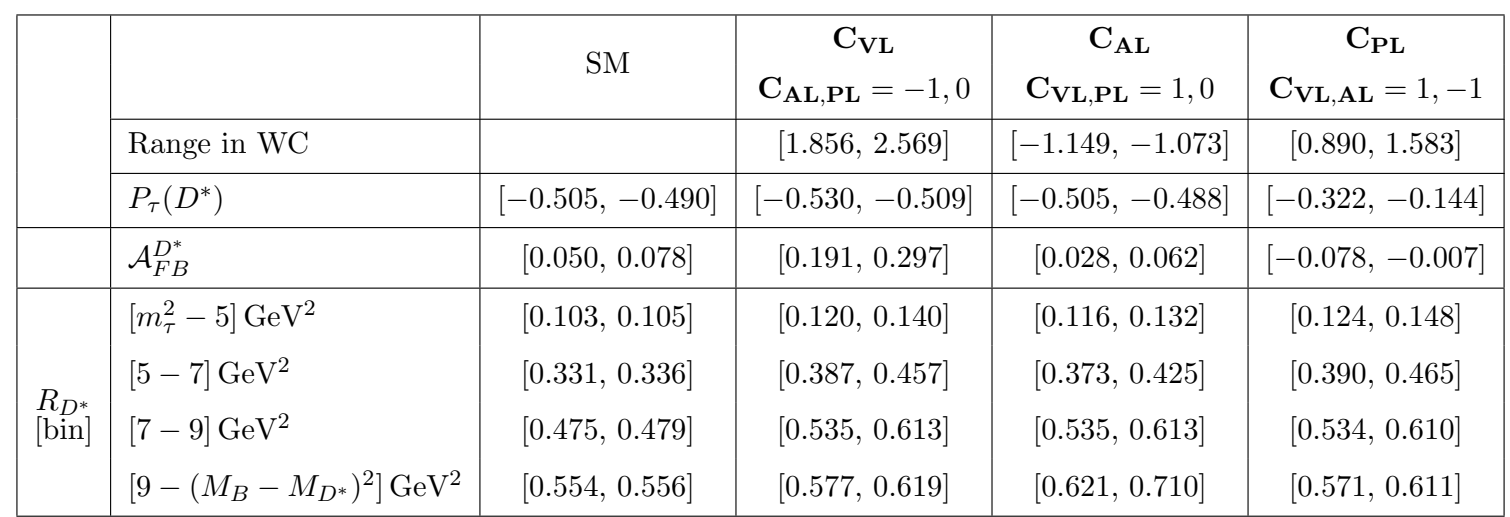

Table 5. The values of the WCs consistent with the $1 \sigma$ experimental range for $R_{D}^{*}$ are shown in the second row. We only show the ranges that are closest to the SM values of the WCs. The subsequent rows show the predictions for $P_{\tau}\left(D^{*}\right), \mathcal{A}_{F B}^{D^{*}}$ and $R_{D^{*}}$ in four $q^{2}$ bins for the WC ranges shown in the second row.
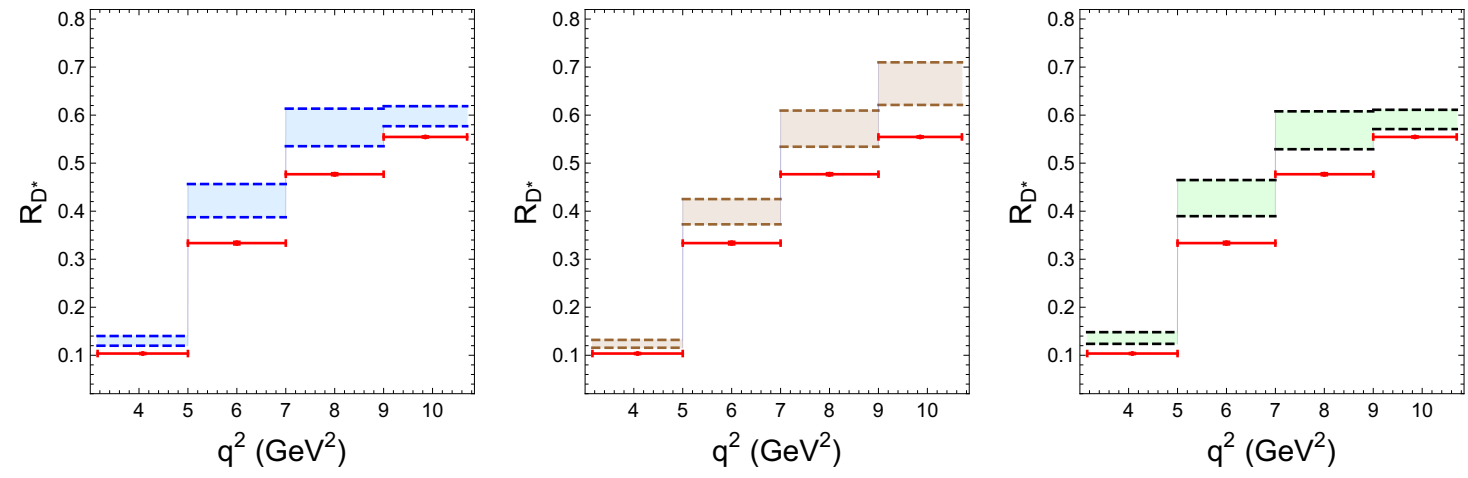

Figure 7. The binwise $R_{D}^{*}$ for four $q^{2}$ bins. On the left, $C_{V L}^{\tau}$ is varied, in the middle $C_{A L}^{\tau}$ is varied, annd on the right, $C_{P L}^{\tau}$ is varied within their $1 \sigma$ allowed ranges. The SM predictions are shown in red.

The binwise $R_{D^{*}}$ values are also plotted in figure 7 . The left, middle and the right panels correspond to the variation of WCs $C_{V L}^{\tau}, C_{A L}^{\tau}$ and $C_{S L}^{\tau}$ respectively. The $1 \sigma$ and $2 \sigma$ experimental values are shown in red and brown respectively. It can be seen that $R_{D^{*}}$ in the last bin can be used to distinguish between $C_{V L}^{\tau}$ (or $\left.C_{P L}^{\tau}\right)$ and $C_{A L}^{\tau}$.

The predictions for $P_{\tau}\left(D^{*}\right)$ are pictorially presented in the left panel of figure 8. We do not show the recent Belle measurements in this figure because the uncertainties are rather large. Instead, we show a projection for Belle II $20 \mathrm{ab}^{-1}$ (which is expected to be collected by the end of 2021 [68]) assuming that the systematic uncertainty will go down by a factor of two compared to that in the recent Belle measurement. It is then possible to distinguish $C_{P L}^{\tau}$ from the other WCs. The middle panel of figure 8 shows the predictions of $\mathcal{A}_{F B}^{D^{*}}$ pictorially. It can be seen that a measurement of $\mathcal{A}_{F B}^{D^{*}}$ can also potentially differentiate the various operators. In the right panel of figure 8 , we show the normalised differential decay width as a function of $q^{2}$ for some representative values of the WCs from table 5. It can be seen that the shape of the distribution does not change dramatically across the various NP explanations of $R_{D^{*}}$. 

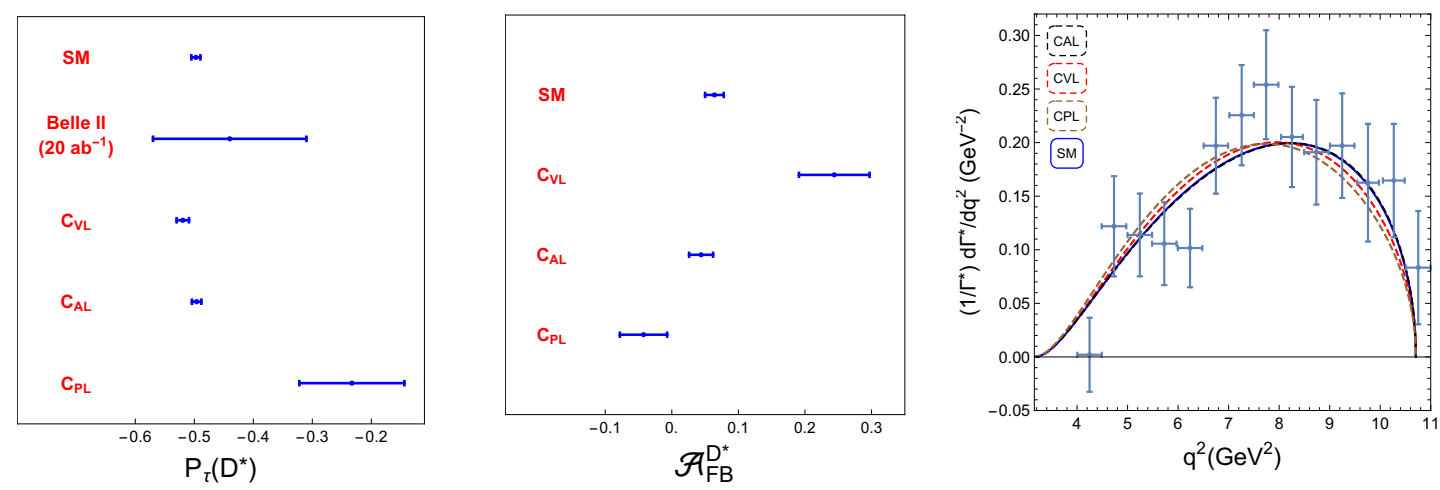

Figure 8. Predictions for the polarisation fraction $P_{\tau}\left(D^{*}\right)$ (left), $\mathcal{A}_{F B}^{D^{*}}$ (middle) and the differential decay width (right). In the left plot, the Belle II $20 \mathrm{ab}^{-1}$ projection is obtained by i) scaling down the statistical uncertainty of the recent Belle measurement by the ratio of the luminosities i.e., $\sqrt{20 / 0.71} \mathrm{ii}$ ) assuming the systematic uncertainty to go down by a factor of two, and adding them in quadrature. The central value is assumed to remain unchanged. On the right plot, The solid blue line is the SM prediction. The dashed black, red and brown lines correspond to $C_{A L}^{\tau}=-1.12$, $C_{V L}^{\tau}=1.9$ and $C_{P L}^{\tau}=1.5$ respectively, where in each case every other $\mathrm{WC}$ is set to their SM values. Note that the black dashed curve is indistinguishable from the SM curve. The data is due to a BaBar measurement reported in [21].
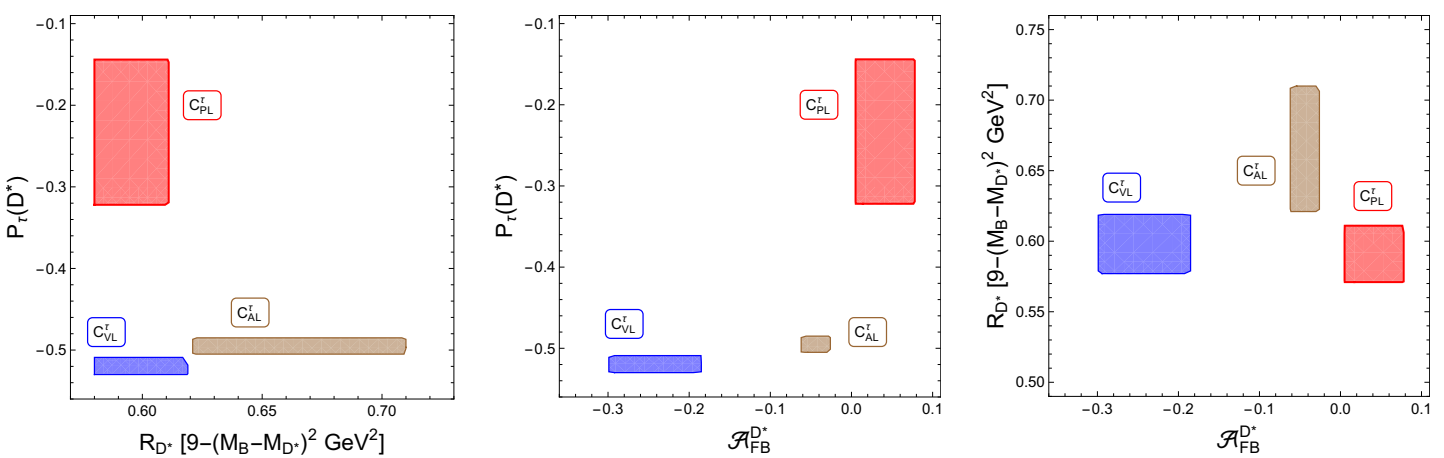

Figure 9. The predictions for $P_{\tau}\left(D^{*}\right), R_{D^{*}}$ in the last bin and $\mathcal{A}_{F B}^{D^{*}}$ are shown in three different planes for the ranges of the three WCs $C_{V L}^{\tau}, C_{A L}^{\tau}$ and $C_{P L}^{\tau}$ given in table 5. We remind the readers that, we have inflated the uncertainties in the form factor parameters in eq. (5.11) by a factor of two. Hence, the ranges of $P_{\tau}\left(D^{*}\right)$ and $R_{D^{*}}$ shown here are rather conservative.

In figure 9 , we show the predictions for $P_{\tau}\left(D^{*}\right), R_{D^{*}}$ in the last bin and $\mathcal{A}_{F B}^{D^{*}}$ in three different planes for the three WCs $C_{V L}^{\tau}, C_{A L}^{\tau}$ and $C_{P L}^{\tau}$ when their values are restricted to the ranges shown in table 5. Interestingly, we find that each of the three pairs of observables can potentially distinguish between the WCs unambiguously. Hence, the measurements of these observables by the experimental collaborations ought to be very much on the cards in their future runs. 


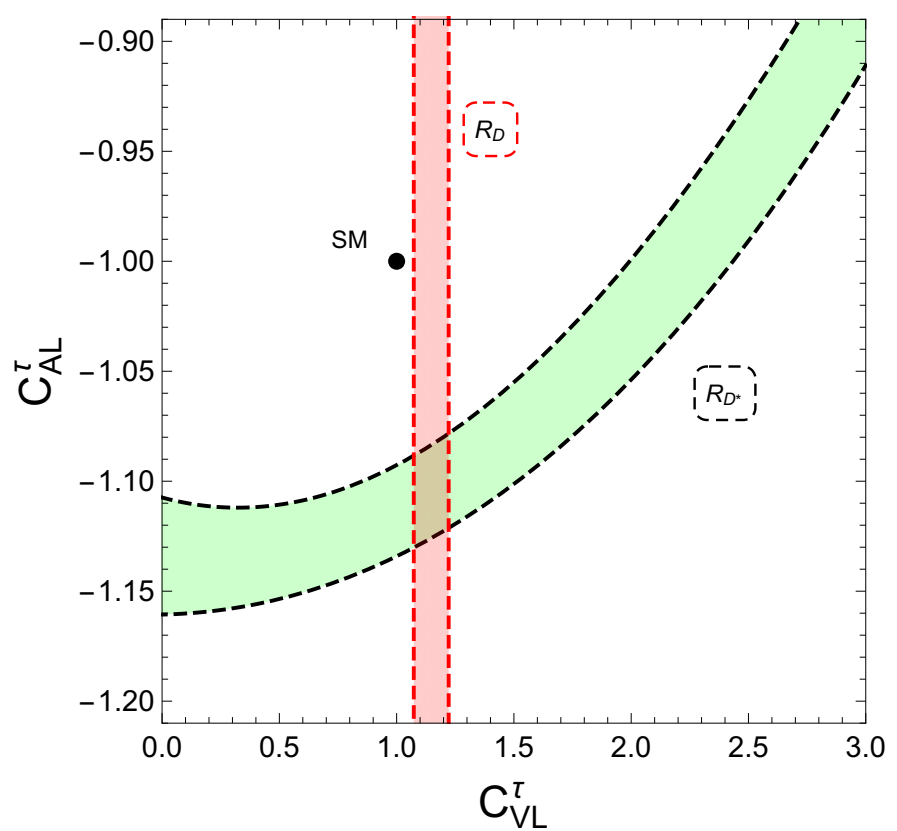

Figure 10. Allowed region in the $C_{V L}^{\tau}-C_{A L}^{\tau}$ plane by $R_{D}$ and $R_{D^{*}}$ measurements .

\subsection{Explaining $R_{D}$ and $R_{D^{*}}$ together}

We have seen from section 8.1 and 8.2 that while $R_{D}$ gets contributions from $C_{V L}^{\tau}$ and $C_{S L}^{\tau}, R_{D^{*}}$ is affected by $C_{V L}^{\tau}, C_{A L}^{\tau}$ and $C_{P L}^{\tau}$. Therefore, in general, these two observables are theoretically independent. In the basis of WCs defined by $\left\{C_{V L}^{\tau}, C_{A L}^{\tau}, C_{S L}^{\tau}, C_{P L}^{\tau}\right\}$, the $C_{V L}^{\tau}$ direction is the only direction that affects both. However, as can be seen from tables 4 and 5, the range of $C_{V L}^{\tau}$ ( i.e., $[1.073,1.222]$ ) that explains $R_{D}$ within $1 \sigma$ is different from the range (i.e., $[1.849,2.648]$ ) that explains $R_{D^{*}}$ successfully within $1 \sigma$. Thus $R_{D}$ and $R_{D^{*}}$ can not be explained simultaneously by invoking NP only of type $C_{V L}^{\tau}$. Figure 10 shows the allowed region in the $C_{V L}^{\tau}-C_{A L}^{\tau}$ plane by the $R_{D}$ and $R_{D^{*}}$ measurements. As $C_{A L}^{\tau}$ does not contribute to the $\bar{B} \rightarrow D \tau \bar{\nu}_{\tau}$ decay, the allowed region for $C_{V L}^{\tau}$ from $R_{D}$ (the red region) is independent of the value of $C_{A L}^{\tau}$. On the other hand, both the WCs $C_{V L}^{\tau}$ and $C_{A L}^{\tau}$ contribute to the $\bar{B} \rightarrow D^{*} \tau \bar{\nu}_{\tau}$ decay and hence the values of these WCs allowed by $R_{D^{*}}$ measurement are correlated. The overlap of the red and the green regions correspond to $C_{V L}^{\tau} \in[1.073,1.222]$ and $C_{A L}^{\tau} \in[-1.144,-1.062]$.

Hence, a minimum value of $C_{V L}^{\tau} \approx-C_{A L}^{\tau} \approx 1.07$ which translates to $\Delta\left(C_{9}-C_{10}\right) \approx 0.15$ (i.e, $15 \%$ shift from the SM values) can explain both $R_{D}$ and $R_{D}^{*}$ successfully. This correspond to the operator $\left[\bar{c} \gamma^{\mu} \mathrm{P}_{L} b\right]\left[\bar{\ell} \gamma_{\mu} \mathrm{P}_{L} \nu\right]$ with a coefficient $g_{N P}^{2} / \Lambda^{2}$ where $\Lambda$ is given by $\Lambda \approx g_{\mathrm{NP}} 2.25 \mathrm{TeV}$.

The predictions for $P_{\tau}\left(D^{*}\right), \mathcal{A}_{F B}^{D^{*}}$ and binwise $R_{D^{*}}$ for the above ranges of $C_{V L}^{\tau}$ and $C_{A L}^{\tau}$ are given in table 6 . 


\begin{tabular}{|c|c|c|c|c|c|}
\hline$C_{V L}^{\tau}$ & $P_{\tau}\left(D^{*}\right)$ & \multicolumn{4}{|c|}{$R_{D^{*}}[\mathrm{bin}]$} \\
\hline$\in[1.073,1.222]$ & $\in[-0.507,-0.489]$ & {$\left[m_{\tau}^{2}-5\right] \mathrm{GeV}^{2}$} & {$[5-7] \mathrm{GeV}^{2}$} & {$[7-9] \mathrm{GeV}^{2}$} & {$\left[9-\left(M_{B}-M_{D^{*}}\right)^{2}\right] \mathrm{GeV}^{2}$} \\
\hline $\begin{array}{c}C_{A L}^{\tau} \\
\in[-1.144,-1.067]\end{array}$ & $\begin{array}{c}\mathcal{A}_{F B}^{D^{*}} \\
\in[0.055,0.092]\end{array}$ & {$[0.116,0.131]$} & {$[0.373,0.426]$} & {$[0.535,0.609]$} & {$[0.616,0.706]$} \\
\hline
\end{tabular}

Table 6. Predictions for $P_{\tau}\left(D^{*}\right), \mathcal{A}_{F B}^{D^{*}}$ and binwise $R_{D^{*}}$ for the values of WCs satisfying both the observations simultaneously. The $1 \sigma$ range of the WCs is given in the first column.

\section{Summary}

In this paper we have performed a model independent analysis of the $R_{D}$ and $R_{D^{*}}$ anomalies using dimension-6 operators that arise in a gauge invariant way. Among the four WCs $C_{V L}^{\tau}$, $C_{A L}^{\tau}, C_{S L}^{\tau}$ and $C_{P L}^{\tau}$, only $C_{V L}^{\tau}$ and $C_{S L}^{\tau}$ contribute to $R_{D}$. On the other hand, $R_{D^{*}}$ gets contributions from $C_{V L}^{\tau}, C_{A L}^{\tau}$ and $C_{P L}^{\tau}$. Thus, $C_{V L}^{\tau}$ is the only WC that affects both (barring tensor operator that is discussed in appendix C) and hence, these two observables are in general theoretically independent. In view of this, initially we studied the solutions of $R_{D}$ and $R_{D^{*}}$ anomalies independent of each other. We obtained the ranges of the WCs that are allowed by the $R_{D}$ and $R_{D}^{*}$ measurements at $1 \sigma$. We also discussed the possibility of simultaneous solutions of these two anomalies.

For the allowed ranges of the WCs, we computed the predictions for both $R_{D}$ and $R_{D^{*}}$ in four different $q^{2}$ bins, the forward-backward asymmetry, $\mathcal{A}_{F B}^{D^{(*)}}$ and the polarisation fraction of the final state $\tau$ lepton. We show that measuring the $\tau$ polarisation in $\bar{B} \rightarrow$ $D^{*} \tau \bar{\nu}_{\tau}$ decays along with the value of $R_{D^{*}}$ in the last $q^{2}$ bin can distinguish between the three WCs which contribute to this process. This is graphically presented in figure 9 . Similarly, as seen in figure 5 , the measurement of the $\tau$ polarisation in $\bar{B} \rightarrow D \tau \bar{\nu}_{\tau}$ decay can in principle be used to distinguish the two WCs $C_{V L}^{\tau}$ and $C_{S L}^{\tau}$. Furthermore, we find that the forward-backward asymmetry of the $\tau$ lepton is also a powerful discriminant of the various WCs (see figures 5 and 9). Unlike $P_{\tau}(D)$, it can even distinguish the sign of the scalar operator for the $\bar{B} \rightarrow D \tau \bar{\nu}_{\tau}$ decay. We hope that the experimental collaborations will take a note of this and make these measurements in near future.

Additionally, in the appendix we also provide the analytic expressions for the double differential decay widths for individual $\tau$ helicities taking into account all the 10 dimension6 operators listed out in section 2 . To our knowledge, we are the first to provide the full expressions in the literature.

Although we have not considered the tensor operator $\mathcal{O}_{\mathrm{TL}}$ in the main text, we have explored its effects on the $R_{D}$ and $R_{D}^{*}$ anomalies in appendix C. We have shown that there exists a small range of $C_{\mathrm{TL}}$ that is consistent with both the anomalies.

\section{Acknowledgments}

We thank Abhishek M. Iyer for collaboration in the very first stage of this work. 
A Full expressions for $a_{\ell}^{D}, b_{\ell}^{D}$ and $c_{\ell}^{D}$

For the negative helicity of the lepton:

$$
\begin{aligned}
& \frac{1}{8} a_{\ell}^{D}(-)=\frac{M_{B}^{2}\left|p_{D}\right|^{2}}{q^{2}}\left|\mathbf{C}_{\mathbf{V L}}^{\ell}\right|^{\mathbf{2}} \mathbf{F}_{+}^{\mathbf{2}}+\frac{\left(M_{B}^{2}-M_{D}^{2}\right)^{2}}{4\left(m_{b}-m_{c}\right)^{2}}\left|\mathbf{C}_{\mathbf{S R}}^{\ell}\right|^{\mathbf{2}} \mathbf{F}_{\mathbf{0}}^{\mathbf{2}} \\
& +m_{\ell}\left[\frac{\left(M_{B}^{2}-M_{D}^{2}\right)^{2}}{2 q^{2}\left(m_{b}-m_{c}\right)} \mathcal{R}\left(\mathbf{C}_{\mathbf{S R}}^{\ell} \mathbf{C}_{\mathbf{V R}}^{\ell *}\right) \mathbf{F}_{\mathbf{0}}^{2}+\frac{4 M_{B}^{2}\left|p_{D}\right|^{2}}{q^{2}\left(M_{B}+M_{D}\right)} \mathcal{R}\left(\mathbf{C}_{\mathbf{T L}}^{\ell} \mathbf{C}_{\mathbf{V L}}^{\ell *}\right) \mathbf{F}_{+} \mathbf{F}_{\mathbf{T}}\right] \\
& +m_{\ell}^{2}\left[\frac{\left(M_{B}^{2}-M_{D}^{2}\right)^{2}}{4 q^{4}}\left|\mathbf{C}_{\mathbf{V R}}^{\ell}\right|^{\mathbf{2}} \mathbf{F}_{\mathbf{0}}^{\mathbf{2}}+\frac{4\left|p_{D}\right|^{2} M_{B}^{2}}{q^{2}\left(M_{B}+M_{D}\right)^{2}}\left|\mathbf{C}_{\mathbf{T L}}^{\ell}\right|^{\mathbf{2}} \mathbf{F}_{\mathbf{T}}^{\mathbf{2}}\right] \\
& \frac{1}{8} b_{\ell}^{D}(-)=\left[-2\left|p_{D}\right| M_{B} \frac{M_{B}-M_{D}}{m_{b}-m_{c}} \mathcal{R}\left(\mathbf{C}_{\mathbf{S R}}^{\ell} \mathbf{C}_{\mathbf{T R}}^{\ell *}\right) \mathbf{F}_{\mathbf{0}} \mathbf{F}_{\mathbf{T}}\right] \\
& -m_{\ell}\left[\frac{2\left|p_{D}\right| M_{B}\left(M_{B}-M_{D}\right)}{q^{2}} \mathcal{R}\left(\mathbf{C}_{\mathbf{V R}}^{\ell} \mathbf{C}_{\mathbf{T R}}^{\ell *}\right) \mathbf{F}_{\mathbf{0}} \mathbf{F}_{\mathbf{T}}\right. \\
& \left.+\frac{\left|p_{D}\right| M_{B}\left(M_{B}^{2}-M_{D}^{2}\right)}{q^{2}\left(m_{b}-m_{c}\right)} \mathcal{R}\left(\mathbf{C}_{\mathbf{S R}}^{\ell} \mathbf{C}_{\mathbf{V R}}^{\ell *}\right) \mathbf{F}_{\mathbf{0}} \mathbf{F}_{+}\right] \\
& -m_{\ell}^{2}\left[\frac{\left|p_{D}\right| M_{B}\left(M_{B}^{2}-M_{D}^{2}\right)}{q^{4}}\left|\mathbf{C}_{\mathbf{V R}}^{\ell}\right|^{\mathbf{2}} \mathbf{F}_{\mathbf{0}} \mathbf{F}_{+}\right] \\
& \frac{1}{8} c_{\ell}^{D}(-)=\left[\frac{4 M_{B}^{2}\left|p_{D}\right|^{2}}{\left(M_{B}+M_{D}\right)^{2}}\left|\mathbf{C}_{\mathbf{T R}}^{\ell}\right|^{\mathbf{2}} \mathbf{F}_{\mathbf{T}}^{\mathbf{2}}-\frac{M_{B}^{2}\left|p_{D}\right|^{2}}{q^{2}}\left|\mathbf{C}_{\mathbf{V L}}^{\ell}\right|^{\mathbf{2}} \mathbf{F}_{+}^{\mathbf{2}}\right] \\
& -m_{\ell}\left[\frac{4\left|p_{D}\right|^{2} M_{B}^{2}}{q^{2}\left(M_{B}+M_{D}\right)}\left(\mathcal{R}\left(\mathbf{C}_{\mathbf{V L}}^{\ell} \mathbf{C}_{\mathbf{T L}}^{\ell}\right) \mathbf{F}_{+} \mathbf{F}_{\mathbf{T}}-\mathcal{R}\left(\mathbf{C}_{\mathbf{V R}}^{\ell} \mathbf{C}_{\mathbf{T R}}^{\ell *}\right) \mathbf{F}_{+} \mathbf{F}_{\mathbf{T}}\right)\right] \\
& +m_{\ell}^{2}\left[\frac{\left|p_{D}\right|^{2} M_{B}^{2}}{q^{4}}\left|\mathbf{C}_{\mathbf{V R}}^{\ell}\right|^{\mathbf{2}} \mathbf{F}_{+}^{2}-\frac{4\left|p_{D}\right|^{2} M_{B}^{2}}{\left(M_{B}+M_{D}\right)^{2} q^{2}}\left|\mathbf{C}_{\mathbf{T L}}^{\ell}\right|^{\mathbf{2}} \mathbf{F}_{\mathbf{T}}^{\mathbf{2}}\right] .
\end{aligned}
$$

For the positive helicity of the lepton:

$$
\begin{aligned}
& \frac{1}{8} a_{\ell}^{D}(+)=\frac{M_{B}^{2}\left|p_{D}\right|^{2}}{q^{2}}\left|\mathbf{C}_{\mathbf{V R}}^{\ell}\right|^{\mathbf{2}} \mathbf{F}_{+}^{\mathbf{2}}+\frac{\left(M_{B}^{2}-M_{D}^{2}\right)^{2}}{4\left(m_{b}-m_{c}\right)^{2}}\left|\mathbf{C}_{\mathbf{S L}}^{\ell}\right|^{\mathbf{2}} \mathbf{F}_{\mathbf{0}}^{\mathbf{2}} \\
& +m_{\ell}\left[\frac{\left(M_{B}^{2}-M_{D}^{2}\right)^{2}}{2 q^{2}\left(m_{b}-m_{c}\right)} \mathcal{R}\left(\mathbf{C}_{\mathbf{S L}}^{\ell} \mathbf{C}_{\mathbf{V L}}^{\ell *}\right) \mathbf{F}_{\mathbf{0}}^{2}+\frac{4 M_{B}^{2}\left|p_{D}\right|^{2}}{q^{2}\left(M_{B}+M_{D}\right)} \mathcal{R}\left(\mathbf{C}_{\mathbf{V R}}^{\ell} \mathbf{C}_{\mathbf{T R}}^{\ell *}\right) \mathbf{F}_{+} \mathbf{F}_{\mathbf{T}}\right] \\
& +m_{\ell}^{2}\left[\frac{\left(M_{B}^{2}-M_{D}^{2}\right)^{2}}{4 q^{4}}\left|\mathbf{C}_{\mathbf{V L}}^{\ell}\right|^{\mathbf{2}} \mathbf{F}_{\mathbf{0}}^{\mathbf{2}}+\frac{4 M_{B}^{2}\left|p_{D}\right|^{2}}{q^{2}\left(M_{B}+M_{D}\right)^{2}}\left|\mathbf{C}_{\mathbf{T R}}^{\ell}\right|^{\mathbf{2}} \mathbf{F}_{\mathbf{T}}^{\mathbf{2}}\right] \\
& \frac{1}{8} b_{\ell}^{D}(+)=\left[-2 M_{B}\left|p_{D}\right| \frac{M_{B}-M_{D}}{m_{b}-m_{c}} \mathcal{R}\left(\mathbf{C}_{\mathbf{S L}}^{\ell} \mathbf{C}_{\mathbf{T L}}^{\ell *}\right) \mathbf{F}_{\mathbf{0}} \mathbf{F}_{\mathbf{T}}\right] \\
& -m_{\ell}\left[\frac{2\left|p_{D}\right|\left(M_{B}-M_{D}\right) M_{B}}{q^{2}} \mathcal{R}\left(\mathbf{C}_{\mathbf{V L}}^{\ell} \mathbf{C}_{\mathbf{T L}}^{\ell *}\right) \mathbf{F}_{\mathbf{0}} \mathbf{F}_{\mathbf{T}}\right. \\
& \left.+\frac{\left|p_{D}\right| M_{B}\left(M_{B}^{2}-M_{D}^{2}\right)}{q^{2}\left(m_{b}-m_{c}\right)} \mathcal{R}\left(\mathbf{C}_{\mathbf{S L}}^{\ell} \mathbf{C}_{\mathbf{V L}}^{\ell *}\right) \mathbf{F}_{\mathbf{0}} \mathbf{F}_{+}\right] \\
& -m_{\ell}^{2}\left[\frac{\left|p_{D}\right| M_{B}\left(M_{B}^{2}-M_{D}^{2}\right)}{q^{4}}\left|\mathbf{C}_{\mathbf{V L}}^{\ell}\right|^{\mathbf{2}} \mathbf{F}_{\mathbf{0}} \mathbf{F}_{+}\right]
\end{aligned}
$$




$$
\begin{aligned}
\frac{1}{8} c_{\ell}^{D}(+)= & {\left[\frac{4\left|p_{D}\right|^{2} M_{B}^{2}}{\left(M_{B}+M_{D}\right)^{2}}\left|\mathbf{C}_{\mathbf{T L}}^{\ell}\right|^{\mathbf{2}} \mathbf{F}_{\mathbf{T}}^{2}-\frac{\left|p_{D}\right|^{2} M_{B}^{2}}{q^{2}}\left|\mathbf{C}_{\mathbf{V R}}^{\ell}\right|^{2} \mathbf{F}_{+}^{2}\right] } \\
& -m_{\ell}\left[\frac{4\left|p_{D}\right|^{2} M_{B}^{2}}{\left(M_{B}+M_{D}\right) q^{2}} \mathcal{R}\left(\mathbf{C}_{\mathbf{V R}}^{\ell} \mathbf{C}_{\mathbf{T R}}^{\ell *}\right) \mathbf{F}_{+} \mathbf{F}_{\mathbf{T}}-\frac{4 M_{B}^{2}\left|p_{D}\right|^{2}}{\left(M_{B}+M_{D}\right) q^{2}} \mathcal{R}\left(\mathbf{C}_{\mathbf{V L}}^{\ell} \mathbf{C}_{\mathbf{T L}}^{\ell *}\right) \mathbf{F}_{+} \mathbf{F}_{\mathbf{T}}\right] \\
& +m_{\ell}^{2}\left[\frac{\left|p_{D}\right|^{2} M_{B}^{2}}{q^{4}}\left|\mathbf{C}_{\mathbf{V L}}^{\ell}\right|^{\mathbf{2} \mathbf{F}_{+}^{2}}-\frac{4\left|p_{D}\right|^{2} M_{B}^{2}}{\left(M_{B}+M_{D}\right)^{2} q^{2}}\left|\mathbf{C}_{\mathbf{T R}}^{\ell}\right|^{\mathbf{2}} \mathbf{F}_{\mathbf{T}}^{2}\right]
\end{aligned}
$$

\section{B Full expressions for $a_{\ell}^{D^{*}}, b_{\ell}^{D^{*}}$ and $c_{\ell}^{D^{*}}$}

$$
\begin{aligned}
& a_{\ell}^{D^{*}}(-) \\
& =\frac{8 M_{B}^{2}\left|p_{D^{*}}\right|^{2}}{\left(M_{B}+M_{D^{*}}\right)^{2}}\left|\mathbf{C}_{\mathbf{V L}}^{\ell}\right|^{\mathbf{2}} \mathbf{V}^{\mathbf{2}}+\frac{\left(M_{B}+M_{D^{*}}\right)^{2}\left(8 M_{D^{*}}^{2} q^{2}+\lambda\right)}{2 M_{D^{*}}^{2} q^{2}}\left|\mathbf{C}_{\mathbf{A L}}^{\ell}\right|^{\mathbf{2}} \mathbf{A}_{\mathbf{1}}^{\mathbf{2}} \\
& +\frac{8 M_{B}^{4}\left|p_{D^{*}}\right|^{4}}{M_{D^{*}}^{2}\left(M_{B}+M_{D^{*}}\right)^{2} q^{2}}\left|\mathbf{C}_{\mathbf{A L}}^{\ell}\right|^{2} \mathbf{A}_{2}^{2} \\
& -\frac{4\left|p_{D^{*}}\right|^{2} M_{B}^{2}\left(M_{B}^{2}-M_{D^{*}}^{2}-q^{2}\right)}{M_{D^{*}}^{2} q^{2}}\left|\mathbf{C}_{\mathbf{A L}}^{\ell}\right|^{2} \mathbf{A}_{\mathbf{1}} \mathbf{A}_{\mathbf{2}} \\
& +\frac{32 M_{B}^{2}\left|p_{D^{*}}\right|^{2}}{q^{2}}\left|\mathbf{C}_{\mathbf{T R}}^{\ell}\right|^{\mathbf{2}} \mathbf{T}_{\mathbf{1}}^{\mathbf{2}}+\frac{8\left(M_{B}^{2}-M_{D^{*}}^{2}\right)^{2}}{q^{2}}\left|\mathbf{C}_{\mathbf{T R}}^{\ell}\right|^{\mathbf{2}} \mathbf{T}_{\mathbf{2}}^{\mathbf{2}} \\
& +m_{\ell}\left[\frac{32 M_{B}^{2}\left|p_{D^{*}}\right|^{2}}{q^{2}\left(M_{B}+M_{D^{*}}\right)} \mathcal{R}\left(\mathbf{C}_{\mathbf{V L}}^{\ell} \mathbf{C}_{\mathbf{T L}}^{\ell *}\right) \mathbf{V} \mathbf{T}_{\mathbf{1}}\right. \\
& +\frac{8\left(M_{B}+M_{D^{*}}\right)\left(2 M_{D^{*}}^{2}\left(M_{B}^{2}-M_{D^{*}}^{2}\right)+M_{B}^{2}\left|p_{D^{*}}\right|^{2}\right)}{q^{2} M_{D^{*}}^{2}} \mathcal{R}\left(\mathbf{C}_{\mathbf{A L}}^{\ell} \mathbf{C}_{\mathbf{T L}}^{\ell *}\right) \mathbf{A}_{\mathbf{1}} \mathbf{T}_{\mathbf{2}} \\
& -\frac{8 M_{B}^{2}\left(M_{B}^{2}-M_{D^{*}}^{2}-q^{2}\right)\left|p_{D^{*}}\right|^{2}}{q^{2}\left(M_{B}-M_{D^{*}}\right) M_{D^{*}}^{2}} \mathcal{R}\left(\mathbf{C}_{\mathbf{A L}}^{\ell} \mathbf{C}_{\mathbf{T L}}^{\ell *}\right) \mathbf{A}_{\mathbf{1}} \mathbf{T}_{\mathbf{3}} \\
& -\frac{8 M_{B}^{2}\left(M_{B}^{2}+3 M_{D^{*}}^{2}-q^{2}\right)\left|p_{D^{*}}\right|^{2}}{q^{2}\left(M_{B}+M_{D^{*}}\right) M_{D^{*}}^{2}} \mathcal{R}\left(\mathbf{C}_{\mathbf{A L}}^{\ell} \mathbf{C}_{\mathbf{T L}}^{\ell *}\right) \mathbf{A}_{\mathbf{2}} \mathbf{T}_{\mathbf{2}} \\
& +\frac{32 M_{B}^{4}\left|p_{D^{*}}\right|^{4}}{q^{2} M_{D^{*}}^{2}\left(M_{B}+M_{D^{*}}\right)\left(M_{B}^{2}-M_{D^{*}}^{2}\right)} \mathcal{R}\left(\mathbf{C}_{\mathbf{A L}}^{\ell} \mathbf{C}_{\mathbf{T L}}^{\ell *}\right) \mathbf{A}_{\mathbf{2}} \mathbf{T}_{\mathbf{3}} \\
& +\frac{32 M_{B}^{2}\left|p_{D^{*}}\right|^{2}}{\left(M_{B}+M_{D^{*}}\right) q^{2}} \mathcal{R}\left(\mathbf{C}_{\mathbf{V R}}^{\ell} \mathbf{C}_{\mathbf{T R}}^{\ell *}\right) \mathbf{V T}_{\mathbf{1}} \\
& \left.-\frac{8\left(M_{B}-M_{D^{*}}\right)\left(M_{B}+M_{D^{*}}\right)^{2}}{q^{2}} \mathcal{R}\left(\mathbf{C}_{\mathbf{A R}}^{\ell} \mathbf{C}_{\mathbf{T R}}^{\ell *}\right) \mathbf{A}_{\mathbf{1}} \mathbf{T}_{\mathbf{2}}\right] \\
& +m_{\ell}^{2}\left[\frac{32 M_{B}^{2}\left|p_{D^{*}}\right|^{2}}{q^{4}}\left|\mathbf{C}_{\mathbf{T L}}^{\ell}\right|^{2} \mathbf{T}_{\mathbf{1}}^{\mathbf{2}}\right. \\
& +\frac{2\left(8 M_{D^{*}}^{2}\left(2\left(M_{B}^{2}+M_{D^{*}}^{2}\right)-q^{2}\right) q^{2}+\left(4 M_{D^{*}}^{2}+q^{2}\right) \lambda\right)}{q^{4} M_{D^{*}}^{2}}\left|\mathbf{C}_{\mathbf{T L}}^{\ell}\right|^{\mathbf{2}} \mathbf{T}_{\mathbf{2}}^{\mathbf{2}} \\
& \left.+\frac{32 M_{B}^{4}\left|p_{D^{*}}\right|^{4}}{q^{2} M_{D^{*}}^{2}\left(M_{B}^{2}-M_{D^{*}}^{2}\right)^{2}}\left|\mathbf{C}_{\mathbf{T L}}^{\ell}\right|^{\mathbf{2}} \mathbf{T}_{\mathbf{3}}^{\mathbf{2}}-\frac{16 M_{B}^{2}\left|p_{D^{*}}\right|^{2}\left(M_{B}^{2}+3 M_{D^{*}}^{2}-q^{2}\right)}{q^{2} M_{D^{*}}^{2}\left(M_{B}^{2}-M_{D^{*}}^{2}\right)}\left|\mathbf{C}_{\mathbf{T L}}^{\ell}\right|^{2} \mathbf{T}_{\mathbf{2}} \mathbf{T}_{\mathbf{3}}\right]
\end{aligned}
$$




$$
\begin{aligned}
& b_{\ell}^{D^{*}}(-)=-16\left|p_{D^{*}}\right| M_{B} \mathcal{R}\left(\mathbf{C}_{\mathbf{V L}}^{\ell} \mathbf{C}_{\mathbf{A} \mathbf{L}}^{\ell *}\right) \mathbf{V A}_{\mathbf{1}} \\
& +\frac{32 M_{B}^{3}\left|p_{D^{*}}\right|^{3}}{\left(m_{b}+m_{c}\right)\left(M_{B}^{2}-M_{D^{*}}^{2}\right) M_{D^{*}}} \mathcal{R}\left(\mathbf{C}_{\mathbf{P R}}^{\ell} \mathbf{C}_{\mathbf{T R}}^{\ell *}\right) \mathbf{A}_{\mathbf{0}} \mathbf{T}_{\mathbf{3}} \\
& -\frac{8 M_{B}\left|p_{D^{*}}\right|\left(M_{B}^{2}+3 M_{D^{*}}^{2}-q^{2}\right)}{\left(m_{b}+m_{c}\right) M_{D^{*}}} \mathcal{R}\left(\mathbf{C}_{\mathbf{P R}}^{\ell} \mathbf{C}_{\mathbf{T R}}^{\ell *}\right) \mathbf{A}_{\mathbf{0}} \mathbf{T}_{\mathbf{2}} \\
& -m_{\ell}\left[\frac{32 M_{B}\left(M_{B}-M_{D^{*}}\right)\left|p_{D^{*}}\right|}{q^{2}} \mathcal{R}\left(\mathbf{C}_{\mathbf{V L}}^{\ell} \mathbf{C}_{\mathbf{T L}}^{\ell *}\right) \mathbf{V T}_{\mathbf{2}}\right. \\
& +\frac{32 M_{B}\left(M_{B}+M_{D^{*}}\right)\left|p_{D^{*}}\right|}{q^{2}} \mathcal{R}\left(\mathbf{C}_{\mathbf{A L}}^{\ell} \mathbf{C}_{\mathbf{T L}}^{\ell *}\right) \mathbf{A}_{\mathbf{1}} \mathbf{T}_{\mathbf{1}} \\
& +\frac{8 M_{B}\left|p_{D^{*}}\right|\left(M_{B}^{2}+3 M_{D^{*}}^{2}-q^{2}\right)}{M_{D^{*}} q^{2}} \mathcal{R}\left(\mathbf{C}_{\mathbf{A R}}^{\ell} \mathbf{C}_{\mathbf{T R}}^{\ell *}\right) \mathbf{A}_{\mathbf{0}} \mathbf{T}_{\mathbf{2}} \\
& \left.-\frac{32 M_{B}^{3}\left|p_{D^{*}}\right|^{3}}{\left(M_{B}-M_{D^{*}}\right) M_{D^{*}}\left(M_{B}+M_{D^{*}}\right) q^{2}} \mathcal{R}\left(\mathbf{C}_{\mathbf{A R}}^{\ell} \mathbf{C}_{\mathbf{T R}}^{\ell *}\right) \mathbf{A}_{\mathbf{0}} \mathbf{T}_{\mathbf{3}}\right] \\
& -m_{\ell}^{2}\left[\frac{64 M_{B}\left(M_{B}^{2}-M_{D^{*}}^{2}\right)\left|p_{D^{*}}\right|}{q^{4}}\left|\mathbf{C}_{\mathbf{T L}}^{\ell}\right|^{\mathbf{2}} \mathbf{T}_{\mathbf{1}} \mathbf{T}_{\mathbf{2}}\right] \\
& c_{\ell}^{D^{*}}(-)=\frac{8\left|p_{D^{*}}\right|^{2} M_{B}^{2}}{\left(M_{B}+M_{D^{*}}\right)^{2}}\left|\mathbf{C}_{\mathbf{V L}}^{\ell}\right|^{2} \mathbf{V}^{\mathbf{2}}-\frac{\left(M_{B}+M_{D^{*}}\right)^{2} \lambda}{2 M_{D^{*}}^{2} q^{2}}\left|\mathbf{C}_{\mathbf{A L}}^{\ell}\right|^{\mathbf{2}} \mathbf{A}_{\mathbf{1}}^{\mathbf{2}} \\
& -\frac{8\left|p_{D^{*}}\right|^{4} M_{B}^{4}}{\left(M_{B}+M_{D^{*}}\right)^{2} M_{D^{*}}^{2} q^{2}}\left|\mathbf{C}_{\mathbf{A L}}^{\ell}\right|^{2} \mathbf{A}_{\mathbf{2}}^{2} \\
& +\frac{4\left|p_{D^{*}}\right|^{2} M_{B}^{2}\left(M_{B}^{2}-M_{D^{*}}^{2}-q^{2}\right)}{M_{D^{*}}^{2} q^{2}}\left|\mathbf{C}_{\mathbf{A L}}^{\ell}\right|^{\mathbf{2}} \mathbf{A}_{\mathbf{1}} \mathbf{A}_{\mathbf{2}} \\
& -\frac{32 M_{B}^{2} M_{D^{*}}^{2}\left(M_{B}^{2}-M_{D^{*}}^{2}\right)^{2}\left|p_{D^{*}}\right|^{2}}{\left(-M_{B}^{2} M_{D^{*}}+M_{D^{*}}^{3}\right)^{2} q^{2}}\left|\mathbf{C}_{\mathbf{T R}}^{\ell}\right|^{2} \mathbf{T}_{\mathbf{1}}^{\mathbf{2}} \\
& \frac{2\left(M_{B}^{2}-M_{D^{*}}^{2}\right)^{2}}{M_{D^{*}}^{2}}\left|\mathbf{C}_{\mathbf{T R}}^{\ell}\right|^{\mathbf{2}} \mathbf{T}_{\mathbf{2}}^{\mathbf{2}} \\
& -\frac{4\left(-M_{B}^{2}+M_{D^{*}}^{2}\right)\left(-M_{B}^{4}+M_{D^{*}}^{4}+4 M_{B}^{2}\left|p_{D^{*}}\right|^{2}\right)}{M_{D^{*}}^{2} q^{2}}\left|\mathbf{C}_{\mathbf{T R}}^{\ell}\right|^{2} \mathbf{T}_{\mathbf{2}}^{2} \\
& +\frac{32 M_{B}^{4}\left|p_{D^{*}}\right|^{4}}{\left(-M_{B}^{2} M_{D^{*}}+M_{D^{*}}^{3}\right)^{2}}\left|\mathbf{C}_{\mathbf{T R}}^{\ell}\right|^{2} \mathbf{T}_{\mathbf{3}}^{\mathbf{2}} \\
& +\frac{16 M_{B}^{2}\left|p_{D^{*}}\right|^{2}\left(M_{B}^{2}+3 M_{D^{*}}^{2}-q^{2}\right)}{-M_{B}^{2} M_{D^{*}}^{2}+M_{D^{*}}^{4}}\left|\mathbf{C}_{\mathbf{T R}}^{\ell}\right|^{\mathbf{2}} \mathbf{T}_{\mathbf{2}} \mathbf{T}_{\mathbf{3}} \\
& +m_{\ell}\left[\frac{32 M_{B}^{2}\left|p_{D^{*}}\right|^{2}}{q^{2}\left(M_{B}+M_{D^{*}}\right)} \mathcal{R}\left(\mathbf{C}_{\mathbf{V L}}^{\ell} \mathbf{C}_{\mathbf{T L}}^{\ell *}\right) \mathbf{V T}_{\mathbf{1}}\right. \\
& -\frac{8 M_{B}^{2}\left(M_{B}+M_{D^{*}}\right)\left|p_{D^{*}}\right|^{2}}{q^{2} M_{D^{*}}^{2}} \mathcal{R}\left(\mathbf{C}_{\mathbf{A L}}^{\ell} \mathbf{C}_{\mathbf{T L}}^{\ell *}\right) \mathbf{A}_{\mathbf{1}} \mathbf{T}_{\mathbf{2}}
\end{aligned}
$$




$$
\begin{aligned}
& +\frac{8 M_{B}^{2}\left(M_{B}^{2}-M_{D^{*}}^{2}-q^{2}\right)\left|p_{D^{*}}\right|^{2}}{q^{2} M_{D^{*}}^{2}\left(M_{B}-M_{D^{*}}\right)} \mathcal{R}\left(\mathbf{C}_{\mathbf{A L}}^{\ell} \mathbf{C}_{\mathbf{T L}}^{\ell *}\right) \mathbf{A}_{\mathbf{1}} \mathbf{T}_{\mathbf{3}} \\
& +\frac{8 M_{B}^{2}\left(M_{B}^{2}+3 M_{D^{*}}^{2}-q^{2}\right)\left|p_{D^{*}}\right|^{2}}{q^{2} M_{D^{*}}^{2}\left(M_{B}+M_{D^{*}}\right)} \mathcal{R}\left(\mathbf{C}_{\mathbf{A L}}^{\ell} \mathbf{C}_{\mathbf{T L}}^{\ell *}\right) \mathbf{A}_{\mathbf{2}} \mathbf{T}_{\mathbf{2}} \\
& -\frac{32 M_{B}^{4}\left|p_{D^{*}}\right|^{4}}{q^{2} M_{D^{*}}^{2}\left(M_{B}+M_{D^{*}}\right)\left(M_{B}^{2}-M_{D^{*}}^{2}\right)} \mathcal{R}\left(\mathbf{C}_{\mathbf{A L}}^{\ell} \mathbf{C}_{\mathbf{T L}}^{\ell *}\right) \mathbf{A}_{\mathbf{2}} \mathbf{T}_{\mathbf{3}} \\
& -\frac{32 M_{B}^{2}\left|p_{D^{*}}\right|^{2}}{\left(M_{B}+M_{D^{*}}\right) q^{2}} \mathcal{R}\left(\mathbf{C}_{\mathbf{V R}}^{\ell} \mathbf{C}_{\mathbf{T R}}^{\ell *}\right) \mathbf{V} \mathbf{T}_{\mathbf{1}} \\
& +\frac{8 M_{B}^{2}\left(M_{B}+M_{D^{*}}\right)\left|p_{D^{*}}\right|^{2}}{M_{D^{*}}^{2} q^{2}} \mathcal{R}\left(\mathbf{C}_{\mathbf{A R}}^{\ell} \mathbf{C}_{\mathbf{T R}}^{\ell *}\right) \mathbf{A}_{\mathbf{1}} \mathbf{T}_{\mathbf{2}} \\
& -\frac{8 M_{B}^{2}\left|p_{D^{*}}\right|^{2}\left(-M_{B}^{2}+M_{D^{*}}^{2}+q^{2}\right)}{\left(M_{B}-M_{D^{*}}\right) M_{D^{*}}^{2} q^{2}} \mathcal{R}\left(\mathbf{C}_{\mathbf{V R}}^{\ell} \mathbf{C}_{\mathbf{T R}}^{\ell *}\right) \mathbf{A}_{\mathbf{1}} \mathbf{T}_{\mathbf{3}} \\
& +\frac{8 M_{B}^{2}\left|p_{D^{*}}\right|^{2}\left(M_{B}^{2}+3 M_{D^{*}}^{2}-q^{2}\right)}{M_{D^{*}}^{2}\left(M_{B}+M_{D^{*}}\right) q^{2}} \mathcal{R}\left(\mathbf{C}_{\mathbf{V R}}^{\ell} \mathbf{C}_{\mathbf{T R}}^{\ell *}\right) \mathbf{A}_{\mathbf{2}} \mathbf{T}_{\mathbf{2}} \\
& \left.-\frac{32 M_{B}^{4}\left|p_{D^{*}}\right|^{4}}{\left(M_{B}-M_{D^{*}}\right) M_{D^{*}}^{2}\left(M_{B}+M_{D^{*}}\right)^{2} q^{2}} \mathcal{R}\left(\mathbf{C}_{\mathbf{V R}}^{\ell} \mathbf{C}_{\mathbf{T R}}^{\ell *}\right) \mathbf{A}_{\mathbf{2}} \mathbf{T}_{\mathbf{3}}\right] \\
& +m_{\ell}^{2}\left[\frac{32 M_{B}^{2}\left|p_{D^{*}}\right|^{2}}{q^{4}}\left|\mathbf{C}_{\mathbf{T L}}^{\ell}\right|^{2} \mathbf{T}_{\mathbf{1}}^{\mathbf{2}}+\frac{2\left(4 M_{D^{*}}^{2}-q^{2}\right) \lambda}{M_{D^{*}}^{2} q^{4}}\left|\mathbf{C}_{\mathbf{T L}}^{\ell}\right|^{\mathbf{2}} \mathbf{T}_{\mathbf{2}}^{\mathbf{2}}\right. \\
& -\frac{32 M_{B}^{4}\left|p_{D^{*}}\right|^{4}}{q^{2} M_{D^{*}}^{2}\left(M_{B}^{2}-M_{D^{*}}^{2}\right)^{2}}\left|\mathbf{C}_{\mathbf{T L}}^{\ell}\right|^{2} \mathbf{T}_{\mathbf{3}}^{\mathbf{2}} \\
& \left.+\frac{16 M_{B}^{2}\left|p_{D^{*}}\right|^{2}\left(M_{B}^{2}+3 M_{D^{*}}^{2}-q^{2}\right)}{q^{2} M_{D^{*}}^{2}\left(M_{B}^{2}-M_{D^{*}}^{2}\right)}\left|\mathbf{C}_{\mathbf{T L}}^{\ell}\right|^{2} \mathbf{T}_{\mathbf{2}} \mathbf{T}_{\mathbf{3}}\right] \\
& a_{\ell}^{D^{*}}(+)=\frac{8\left|p_{D^{*}}\right|^{2} M_{B}^{2}}{\left(m_{b}+m_{c}\right)^{2}}\left|\mathbf{C}_{\mathbf{P L}}^{\ell}\right|^{2} \mathbf{A}_{\mathbf{0}}^{2}+\frac{32 M_{B}^{2}\left|p_{D^{*}}\right|^{2}}{q^{2}}\left|\mathbf{C}_{\mathbf{T L}}^{\ell}\right|^{2} \mathbf{T}_{\mathbf{1}}^{2}+\frac{8\left(M_{B}^{2}-M_{D^{*}}^{2}\right)^{2}}{q^{2}}\left|\mathbf{C}_{\mathbf{T L}}^{\ell}\right|^{2} \mathbf{T}_{\mathbf{2}}^{\mathbf{2}} \\
& -m_{\ell}\left[\frac{16\left|p_{D^{*}}\right|^{2} M_{B}^{2}}{\left(m_{b}+m_{c}\right) q^{2}} \mathcal{R}\left(\mathbf{C}_{\mathbf{A L}}^{\ell} \mathbf{C}_{\mathbf{P L}}^{\ell *}\right) \mathbf{A}_{\mathbf{0}}^{2}\right. \\
& -\frac{32 M_{B}^{2}\left|p_{D^{*}}\right|^{2}}{q^{2}\left(M_{B}+M_{D^{*}}\right)} \mathcal{R}\left(\mathbf{C}_{\mathbf{V L}}^{\ell} \mathbf{C}_{\mathbf{T L}}^{\ell *}\right) \mathbf{V} \mathbf{T}_{\mathbf{1}} \\
& -\frac{8\left(M_{B}+M_{D^{*}}\right)\left(M_{B}^{2}-M_{D^{*}}^{2}\right)}{q^{2}} \mathcal{R}\left(\mathbf{C}_{\mathbf{A L}}^{\ell} \mathbf{C}_{\mathbf{T L}}^{\ell *}\right) \mathbf{A}_{\mathbf{1}} \mathbf{T}_{\mathbf{2}} \\
& -\frac{32 M_{B}^{2}\left|p_{D^{*}}\right|^{2}}{\left(M_{B}+M_{D^{*}}\right) q^{2}} \mathcal{R}\left(\mathbf{C}_{\mathbf{V R}}^{\ell} \mathbf{C}_{\mathbf{T R}}^{\ell *}\right) \mathbf{V} \mathbf{T}_{\mathbf{1}} \\
& +\frac{8\left(M_{B}+M_{D^{*}}\right)\left(-2 M_{D^{*}}^{4}+M_{B}^{2}\left(2 M_{D^{*}}^{2}+\left|p_{D^{*}}\right|^{2}\right)\right)}{M_{D^{*}}^{2} q^{2}} \mathcal{R}\left(\mathbf{C}_{\mathbf{A R}}^{\ell} \mathbf{C}_{\mathbf{T R}}^{\ell *}\right) \mathbf{A}_{\mathbf{1}} \mathbf{T}_{\mathbf{2}} \\
& +\frac{8 M_{B}^{2}\left|p_{D^{*}}\right|^{2}\left(-M_{B}^{2}+M_{D^{*}}^{2}+q^{2}\right)}{\left(M_{B}-M_{D^{*}}\right) M_{D^{*}}^{2} q^{2}} \mathcal{R}\left(\mathbf{C}_{\mathbf{A R}}^{\ell} \mathbf{C}_{\mathbf{T R}}^{\ell *}\right) \mathbf{A}_{\mathbf{1}} \mathbf{T}_{\mathbf{3}}
\end{aligned}
$$




$$
\begin{aligned}
& -\frac{8 M_{B}^{2}\left|p_{D^{*}}\right|^{2}\left(M_{B}^{2}+3 M_{D^{*}}^{2}-q^{2}\right)}{M_{D^{*}}^{2}\left(M_{B}+M_{D^{*}}\right) q^{2}} \mathcal{R}\left(\mathbf{C}_{\mathbf{A R}}^{\ell} \mathbf{C}_{\mathbf{T R}}^{\ell *}\right) \mathbf{A}_{\mathbf{2}} \mathbf{T}_{\mathbf{2}} \\
& \left.+\frac{32 M_{B}^{4}\left|p_{D^{*}}\right|^{4}}{\left(M_{B}-M_{D^{*}}\right) M_{D^{*}}^{2}\left(M_{B}+M_{D^{*}}\right)^{2} q^{2}} \mathcal{R}\left(\mathbf{C}_{\mathbf{A R}}^{\ell} \mathbf{C}_{\mathbf{T R}}^{\ell *}\right) \mathbf{A}_{\mathbf{2}} \mathbf{T}_{\mathbf{3}}\right] \\
& +m_{\ell}^{2}\left[\frac{8\left|p_{D^{*}}\right|^{2} M_{B}^{2}}{q^{4}}\left|\mathbf{C}_{\mathbf{A L}}^{\ell}\right|^{\mathbf{2}} \mathbf{A}_{\mathbf{0}}^{\mathbf{2}}+\frac{8\left|p_{D^{*}}\right|^{2} M_{B}^{2}}{\left(M_{B}+M_{D^{*}}\right)^{2} q^{2}}\left|\mathbf{C}_{\mathbf{V L}}^{\ell}\right|^{\mathbf{2}} \mathbf{V}^{\mathbf{2}}\right. \\
& +\frac{2\left(M_{B}+M_{D^{*}}\right)^{2}}{q^{2}}\left|\mathbf{C}_{\mathbf{A L}}^{\ell}\right|^{2} \mathbf{A}_{\mathbf{1}}^{2} \\
& +\frac{32 M_{B}^{2}\left|p_{D^{*}}\right|^{2}}{q^{4}}\left|\mathbf{C}_{\mathbf{T R}}^{\ell}\right|^{2} \mathbf{T}_{\mathbf{1}}^{\mathbf{2}}+8 \frac{M_{B}^{2}\left|p_{D^{*}}\right|^{2}}{M_{D^{*}}^{2} q^{2}}\left|\mathbf{C}_{\mathbf{T R}}^{\ell}\right|^{2} \mathbf{T}_{\mathbf{2}}^{\mathbf{2}} \\
& +\frac{16\left(M_{B}^{4}+M_{D^{*}}^{4}-2 M_{B}^{2}\left(M_{D^{*}}^{2}+\left|p_{D^{*}}\right|^{2}\right)\right)}{q^{4}}\left|\mathbf{C}_{\mathbf{T R}}^{\ell}\right|^{2} \mathbf{T}_{\mathbf{2}}^{\mathbf{2}} \\
& +\frac{32 M_{B}^{4}\left|p_{D^{*}}\right|^{4}}{\left(-M_{B}^{2} M_{D^{*}}+M_{D^{*}}^{3}\right)^{2} q^{2}}\left|\mathbf{C}_{\mathbf{T R}}^{\ell}\right|^{\mathbf{2}} \mathbf{T}_{\mathbf{3}}^{\mathbf{2}} \\
& \left.+\frac{16 M_{B}^{2}\left|p_{D^{*}}\right|^{2}\left(M_{B}^{2}+3 M_{D^{*}}^{2}-q^{2}\right)}{M_{D^{*}}^{2}\left(-M_{B}^{2}+M_{D^{*}}^{2}\right) q^{2}}\left|\mathbf{C}_{\mathbf{T R}}^{\ell}\right|^{2} \mathbf{T}_{\mathbf{2}} \mathbf{T}_{\mathbf{3}}\right] \\
& b_{\ell}^{D^{*}}(+)=\frac{8 M_{B}\left(M_{B}^{2}+3 M_{D^{*}}^{2}-q^{2}\right)\left|p_{D^{*}}\right|}{\left(m_{b}+m_{c}\right) M_{D^{*}}} \mathcal{R}\left(\mathbf{C}_{\mathbf{P L}}^{\ell} \mathbf{C}_{\mathbf{T L}}^{\ell *}\right) \mathbf{A}_{\mathbf{0}} \mathbf{T}_{\mathbf{2}} \\
& -\frac{32 M_{B}^{3}\left|p_{D^{*}}\right|^{3}}{\left(m_{b}+m_{c}\right) M_{D^{*}}\left(M_{B}^{2}-M_{D^{*}}^{2}\right)} \mathcal{R}\left(\mathbf{C}_{\mathbf{P L}}^{\ell} \mathbf{C}_{\mathbf{T L}}^{\ell *}\right) \mathbf{A}_{\mathbf{0}} \mathbf{T}_{\mathbf{3}} \\
& +m_{\ell}\left[\frac{4\left|p_{D^{*}}\right| M_{B}\left(M_{B}+M_{D^{*}}\right)\left(M_{B}^{2}-M_{D^{*}}^{2}-q^{2}\right)}{M_{D^{*}}\left(m_{b}+m_{c}\right) q^{2}} \mathcal{R}\left(\mathbf{C}_{\mathbf{A L}}^{\ell} \mathbf{C}_{\mathbf{P L}}^{\ell *}\right) \mathbf{A}_{\mathbf{0}} \mathbf{A}_{\mathbf{1}}\right. \\
& -\frac{16}{\left(m_{b}+m_{c}\right)} \frac{\left|p_{D^{*}}\right|^{3} M_{B}^{3}}{\left(M_{B}+M_{D^{*}}\right) M_{D^{*}} q^{2}} \mathcal{R}\left(\mathbf{C}_{\mathbf{A L}}^{\ell} \mathbf{C}_{\mathbf{P L}}^{\ell *}\right) \mathbf{A}_{\mathbf{0}} \mathbf{A}_{\mathbf{2}} \\
& -\frac{8 M_{B}\left(M_{B}^{2}+3 M_{D^{*}}^{2}-q^{2}\right)\left|p_{D^{*}}\right|}{M_{D^{*}} q^{2}} \mathcal{R}\left(\mathbf{C}_{\mathbf{A L}}^{\ell} \mathbf{C}_{\mathbf{T L}}^{\ell *}\right) \mathbf{A}_{\mathbf{0}} \mathbf{T}_{\mathbf{2}} \\
& +\frac{32 M_{B}^{3}\left|p_{D^{*}}\right|^{3}}{q^{2} M_{D^{*}}\left(M_{B}^{2}-M_{D^{*}}^{2}\right)} \mathcal{R}\left(\mathbf{C}_{\mathbf{A L}}^{\ell} \mathbf{C}_{\mathbf{T L}}^{\ell *}\right) \mathbf{A}_{\mathbf{0}} \mathbf{T}_{\mathbf{3}} \\
& +\frac{32 M_{B}\left(-M_{B}+M_{D^{*}}\right)\left|p_{D^{*}}\right|}{q^{2}} \mathcal{R}\left(\mathbf{C}_{\mathbf{V R}}^{\ell} \mathbf{C}_{\mathbf{T R}}^{\ell *}\right) \mathbf{V} \mathbf{T}_{\mathbf{2}} \\
& \left.+\frac{32 M_{B}\left(M_{B}+M_{D^{*}}\right)\left|p_{D^{*}}\right|}{q^{2}} \mathcal{R}\left(\mathbf{C}_{\mathbf{A R}}^{\ell} \mathbf{C}_{\mathbf{T R}}^{\ell *}\right) \mathbf{A}_{\mathbf{1}} \mathbf{T}_{\mathbf{1}}\right] \\
& +m_{\ell}^{2}\left[-\frac{4\left|p_{D^{*}}\right| M_{B}\left(M_{B}+M_{D^{*}}\right)}{M_{D^{*}} q^{4}}\left(M_{B}^{2}-M_{D^{*}}^{2}-q^{2}\right)\left|\mathbf{C}_{\mathbf{A L}}^{\ell}\right|^{2} \mathbf{A}_{\mathbf{0}} \mathbf{A}_{\mathbf{1}}\right. \\
& +\frac{16\left|p_{D^{*}}\right|^{3} M_{B}^{3}}{\left(M_{B}+M_{D^{*}}\right) M_{D^{*}} q^{4}}\left|\mathbf{C}_{\mathbf{A L}}^{\ell}\right|^{2} \mathbf{A}_{\mathbf{0}} \mathbf{A}_{\mathbf{2}} \\
& \left.+\frac{64 M_{B}\left(-M_{B}^{2}+M_{D^{*}}^{2}\right)\left|p_{D^{*}}\right|}{q^{4}}\left|\mathbf{C}_{\mathbf{T R}}^{\ell}\right|^{2} \mathbf{T}_{\mathbf{1}} \mathbf{T}_{\mathbf{2}}\right]
\end{aligned}
$$




$$
\begin{aligned}
& c_{\ell}^{D^{*}}(+)=-\frac{32 M_{B}^{2}\left|p_{D^{*}}\right|^{2}}{q^{2}}\left|\mathbf{C}_{\mathbf{T L}}^{\ell}\right|^{\mathbf{2}} \mathbf{T}_{\mathbf{1}}^{\mathbf{2}}-\frac{2\left(4 M_{D^{*}}^{2}-q^{2}\right) \lambda}{M_{D^{*}}^{2} q^{2}}\left|\mathbf{C}_{\mathbf{T L}}^{\ell}\right|^{\mathbf{2}} \mathbf{T}_{\mathbf{2}}^{\mathbf{2}} \\
& +\frac{32 M_{B}^{4}\left|p_{D^{*}}\right|^{4}}{M_{D^{*}}^{2}\left(M_{B}^{2}-M_{D^{*}}^{2}\right)^{2}}\left|\mathbf{C}_{\mathbf{T L}}^{\ell}\right|^{2} \mathbf{T}_{\mathbf{3}}^{2} \\
& -\frac{16 M_{B}^{2}\left|p_{D^{*}}\right|^{2}\left(M_{B}^{2}+3 M_{D^{*}}^{2}-q^{2}\right)}{M_{D^{*}}^{2}\left(M_{B}^{2}-M_{D^{*}}^{2}\right)}\left|\mathbf{C}_{\mathbf{T L}}^{\ell}\right|^{2} \mathbf{T}_{\mathbf{2}} \mathbf{T}_{\mathbf{3}} \\
& -m_{\ell}\left[\frac{32 M_{B}^{2}\left|p_{D^{*}}\right|^{2}}{q^{2}\left(M_{B}+M_{D^{*}}\right)} \mathcal{R}\left(\mathbf{C}_{\mathbf{V L}}^{\ell} \mathbf{C}_{\mathbf{T L}}^{\ell *}\right) \mathbf{V} \mathbf{T}_{\mathbf{1}}\right. \\
& -\frac{8 M_{B}^{2}\left(M_{B}+M_{D^{*}}\right)\left|p_{D^{*}}\right|^{2}}{q^{2} M_{D^{*}}^{2}} \mathcal{R}\left(\mathbf{C}_{\mathbf{A L}}^{\ell} \mathbf{C}_{\mathbf{T L}}^{\ell *}\right) \mathbf{A}_{\mathbf{1}} \mathbf{T}_{\mathbf{2}} \\
& +\frac{8 M_{B}^{2}\left(M_{B}^{2}-M_{D^{*}}^{2}-q^{2}\right)\left|p_{D^{*}}\right|^{2}}{q^{2} M_{D^{*}}^{2}\left(M_{B}-M_{D^{*}}\right)} \mathcal{R}\left(\mathbf{C}_{\mathbf{A L}}^{\ell} \mathbf{C}_{\mathbf{T L}}^{\ell *}\right) \mathbf{A}_{\mathbf{1}} \mathbf{T}_{\mathbf{3}} \\
& +\frac{8 M_{B}^{2}\left(M_{B}^{2}+3 M_{D^{*}}^{2}-q^{2}\right)\left|p_{D^{*}}\right|^{2}}{q^{2} M_{D^{*}}^{2}\left(M_{B}+M_{D^{*}}\right)} \mathcal{R}\left(\mathbf{C}_{\mathbf{A L}}^{\ell} \mathbf{C}_{\mathbf{T L}}^{\ell *}\right) \mathbf{A}_{\mathbf{2}} \mathbf{T}_{\mathbf{2}} \\
& -\frac{32 M_{B}^{4}\left|p_{D^{*}}\right|^{4}}{q^{2} M_{D^{*}}^{2}\left(M_{B}+M_{D^{*}}\right)\left(M_{B}^{2}-M_{D^{*}}^{2}\right)} \mathcal{R}\left(\mathbf{C}_{\mathbf{A L}}^{\ell} \mathbf{C}_{\mathbf{T L}}^{\ell *}\right) \mathbf{A}_{\mathbf{2}} \mathbf{T}_{\mathbf{3}} \\
& +\frac{32 M_{B}^{2}\left|p_{D^{*}}\right|^{2}}{\left(M_{B}+M_{D^{*}}\right) q^{2}} \mathcal{R}\left(\mathbf{C}_{\mathbf{V R}}^{\ell} \mathbf{C}_{\mathbf{T R}}^{\ell *}\right) \mathbf{V} \mathbf{T}_{\mathbf{1}} \\
& +\frac{8 M_{B}^{2}\left(M_{B}+M_{D^{*}}\right)\left|p_{D^{*}}\right|^{2}}{M_{D^{*}}^{2} q^{2}} \mathcal{R}\left(\mathbf{C}_{\mathbf{A R}}^{\ell} \mathbf{C}_{\mathbf{T R}}^{\ell *}\right) \mathbf{A}_{\mathbf{1}} \mathbf{T}_{\mathbf{2}} \\
& -\frac{8 M_{B}^{2}\left|p_{D^{*}}\right|^{2}\left(-M_{B}^{2}+M_{D^{*}}^{2}+q^{2}\right)}{\left(M_{B}-M_{D^{*}}\right) M_{D^{*}}^{2} q^{2}} \mathcal{R}\left(\mathbf{C}_{\mathbf{A R}}^{\ell} \mathbf{C}_{\mathbf{T R}}^{\ell *}\right) \mathbf{A}_{\mathbf{1}} \mathbf{T}_{\mathbf{3}} \\
& +\frac{8 M_{B}^{2}\left|p_{D^{*}}\right|^{2}\left(M_{B}^{2}+3 M_{D^{*}}^{2}-q^{2}\right)}{M_{D^{*}}^{2}\left(M_{B}+M_{D^{*}}\right) q^{2}} \mathcal{R}\left(\mathbf{C}_{\mathbf{A R}}^{\ell} \mathbf{C}_{\mathbf{T R}}^{\ell *}\right) \mathbf{A}_{\mathbf{2}} \mathbf{T}_{\mathbf{2}} \\
& \left.-\frac{32 M_{B}^{4}\left|p_{D^{*}}\right|^{4}}{\left(M_{B}-M_{D^{*}}\right) M_{D^{*}}^{2}\left(M_{B}+M_{D^{*}}\right)^{2} q^{2}} \mathcal{R}\left(\mathbf{C}_{\mathbf{A R}}^{\ell} \mathbf{C}_{\mathbf{T R}}^{\ell *}\right) \mathbf{A}_{\mathbf{2}} \mathbf{T}_{\mathbf{3}}\right] \\
& +m_{\ell}^{2}\left[-\frac{8\left|p_{D^{*}}\right|^{2} M_{B}^{2}}{\left(M_{B}+M_{D^{*}}\right)^{2} q^{2}}\left|\mathbf{C}_{\mathbf{V L}}^{\ell}\right|^{\mathbf{2}} \mathbf{V}^{\mathbf{2}}+\frac{\left(M_{B}+M_{D^{*}}\right)^{2} \lambda}{2 M_{D^{*}}^{2} q^{4}}\left|\mathbf{C}_{\mathbf{A L}}^{\ell}\right|^{\mathbf{2}} \mathbf{A}_{\mathbf{1}}^{\mathbf{2}}\right. \\
& +\frac{8\left|p_{D^{*}}\right|^{4} M_{B}^{4}}{M_{D^{*}}^{2}\left(M_{B}+M_{D^{*}}\right)^{2} q^{4}}\left|\mathbf{C}_{\mathbf{A L}}^{\ell}\right|^{2} \mathbf{A}_{\mathbf{2}}^{2} \\
& -\frac{4\left|p_{D^{*}}\right|^{2} M_{B}^{2}}{M_{D^{*}}^{2} q^{4}}\left(M_{B}^{2}-M_{D^{*}}^{2}-q^{2}\right)\left|\mathbf{C}_{\mathbf{A L}}^{\ell}\right|^{2} \mathbf{A}_{\mathbf{1}} \mathbf{A}_{\mathbf{2}} \\
& +\frac{32 M_{B}^{2}\left|p_{D^{*}}\right|^{2}}{q^{4}}\left|\mathbf{C}_{\mathbf{T R}}^{\ell}\right|^{\mathbf{2}} \mathbf{T}_{\mathbf{1}}^{\mathbf{2}}+\frac{8 M_{B}^{2}\left|p_{D^{*}}\right|^{2}\left(4 M_{D^{*}}^{2}-q^{2}\right)}{M_{D^{*}}^{2} q^{4}}\left|\mathbf{C}_{\mathbf{T R}}^{\ell}\right|^{\mathbf{2}} \mathbf{T}_{\mathbf{2}}^{\mathbf{2}} \\
& -\frac{32 M_{B}^{4}\left|p_{D^{*}}\right|^{4}}{\left(-M_{B}^{2} M_{D^{*}}+M_{D^{*}}^{3}\right)^{2} q^{2}}\left|\mathbf{C}_{\mathbf{T R}}^{\ell}\right|^{2} \mathbf{T}_{\mathbf{3}}^{2} \\
& \left.+\frac{16 M_{B}^{2}\left|p_{D^{*}}\right|^{2}\left(M_{B}^{2}+3 M_{D^{*}}^{2}-q^{2}\right)}{M_{D^{*}}^{2}\left(M_{B}^{2}-M_{D^{*}}^{2}\right) q^{2}}\left|\mathbf{C}_{\mathbf{T R}}^{\ell}\right|^{2} \mathbf{T}_{\mathbf{2}} \mathbf{T}_{\mathbf{3}}\right]
\end{aligned}
$$




\begin{tabular}{|c|c|c|c|c|c|}
\hline \multirow{2}{*}{$\begin{array}{c}C_{T L}^{\tau} \in[0.240,0.796] \\
C_{T L}^{\tau}\end{array}$} & $P_{\tau}(D)$ & \multicolumn{4}{|c|}{$R_{D}[\mathrm{bin}]$} \\
\cline { 3 - 6 } & $\in[0.125,0.254]$ & {$\left[m_{\tau}^{2}-5\right] \mathrm{GeV}^{2}$} & {$[5-7] \mathrm{GeV}^{2}$} & {$[7-9] \mathrm{GeV}^{2}$} & {$\left[9-\left(M_{B}-M_{D}\right)^{2}\right] \mathrm{GeV}^{2}$} \\
\cline { 3 - 6 } & $\mathcal{A}_{F B}^{D}$ & {$[0.178,0.233]$} & {$[0.673,0.907]$} & {$[1.135,1.533]$} & {$[1.989,2.508]$} \\
\hline
\end{tabular}

Table 7. Predictions for $P_{\tau}(D), \mathcal{A}_{F B}^{D}$ and binwise values of $R_{D}$ for a range of $C_{T L}^{\tau}$ for which $R_{D}$ is experimentally satisfied within $1 \sigma$. The range of the WCs is given in the first column. The values in the subsequent columns are only for the range of $C_{T L}^{\tau}$ closest to the SM value of 0 , viz. the positive range.
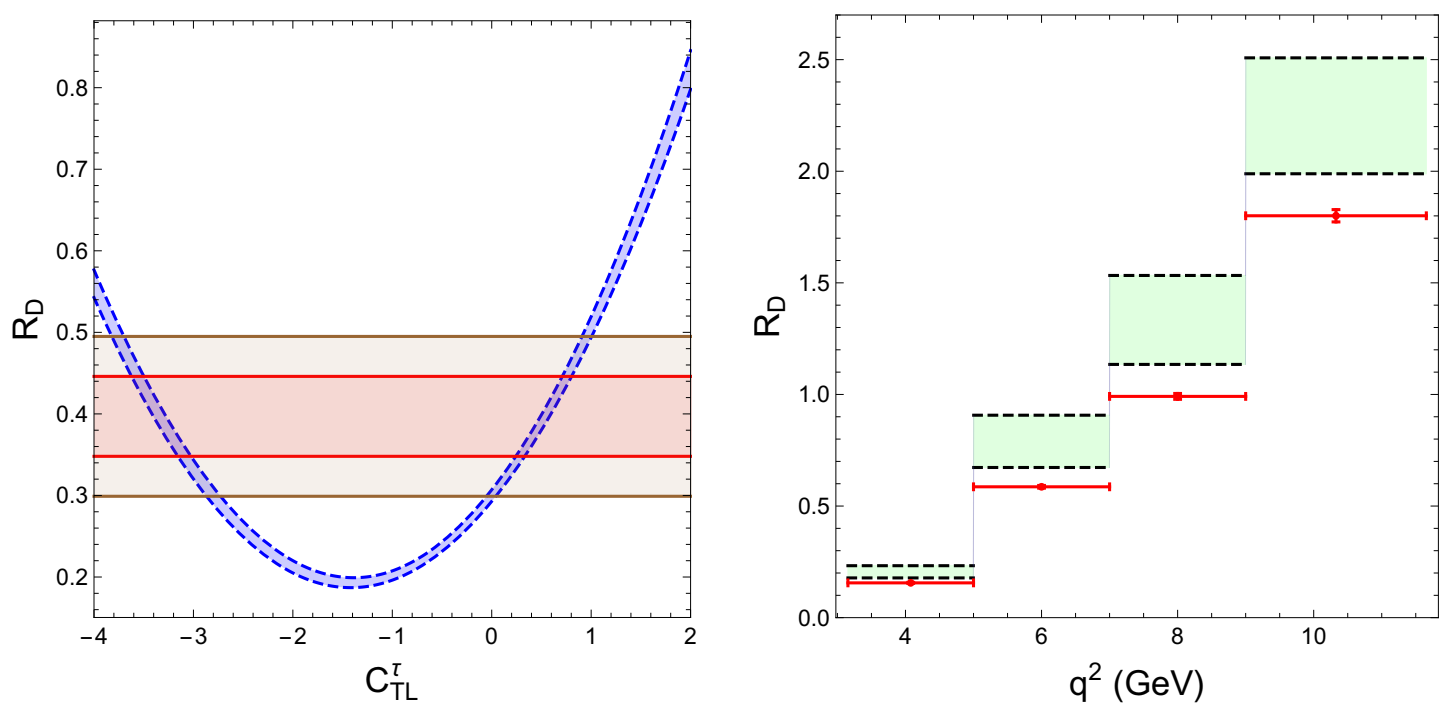

Figure 11. The left panel shows the dependence of $R_{D}$ with respect to the variation of the WCs $C_{T L}^{\tau}$ and the right panel shows the prediction for $R_{D}$ in four different bins of $q^{2}$ from table 7 .

\section{Contribution of the Tensor operator $\mathcal{O}_{\mathrm{TL}}^{c b \ell}$}

\section{C.1 $\quad \bar{B} \rightarrow D \tau \bar{\nu}_{\tau}$}

In this section we investigate the effect of the tensor operator $\mathcal{O}_{\mathrm{TL}}^{\text {cbl }}$ on the $\bar{B} \rightarrow D \tau \bar{\nu}_{\tau}$ decay. In the first column of table 7 , we show the range of $C_{T L}^{\tau}$ that explains $R_{D}$ within $1 \sigma$. In the subsequent columns, we show the predictions of $P_{\tau}(D), \mathcal{A}_{F B}^{D}$ and binwise $R_{D}$ for the allowed range of $C_{T L}^{\tau}$ that is closest to zero (i.e., $\left.C_{T L}^{\tau} \in[0.240,0.796]\right)$. A comparison with the left plot of figure 6 reveals that $P_{\tau}(D)$ in this case is quite different from the other cases and thus, can completely distinguish the tensor operator from the vector or scalar operators. Similarly, $\mathcal{A}_{F B}^{D}$ can also be used to distinguish the tensor from the vector operator, however, there exists some degeneracy with the scalar operator.

The variation of $R_{D}$ as a function of $C_{T L}^{\tau}$ is also shown in the left plot of figure 11 . The predictions for binwise $R_{D}$ for the tensor operators are graphically presented in the right plot of figure 11 . 


\begin{tabular}{|c|c|c|c|c|c|}
\hline \multirow{2}{*}{$\begin{array}{c}C_{T L}^{\tau} \\
\in[-0.120,-0.058]\end{array}$} & $P_{\tau}\left(D^{*}\right)$ & \multicolumn{4}{|c|}{$R_{D^{*}}[\mathrm{bin}]$} \\
\cline { 3 - 6 } & $\in[-0.481,-0.441]$ & {$\left[m_{\tau}^{2}-5\right] \mathrm{GeV}^{2}$} & {$[5-7] \mathrm{GeV}^{2}$} & {$[7-9] \mathrm{GeV}^{2}$} & {$\left[9-\left(M_{B}-M_{D^{*}}\right)^{2}\right] \mathrm{GeV}^{2}$} \\
\cline { 3 - 6 }$C_{T L}^{\tau}$ & $\mathcal{A}_{F B}^{D^{*}}$ & {$[0.113,0.129]$} & {$[0.368,0.423]$} & {$[0.531,0.610]$} & {$[0.620,0.715]$} \\
\hline$[0.709,0.834]$ & $\in[-0.016,0.034]$ & & & & \\
\hline
\end{tabular}

Table 8. Predictions for $P_{\tau}\left(D^{*}\right), \mathcal{A}_{F B}^{D^{*}}$ and binwise values of $R_{D^{*}}$ for a range of $C_{T L}^{\tau}$ for which $R_{D^{*}}$ is experimentally satisfied within $1 \sigma$. The corresponding range of the WCs is given in the first column. The values in the subsequent columns are only for the range of $C_{T L}^{\tau}$ closest to the SM value of 0 , viz. the negative range.
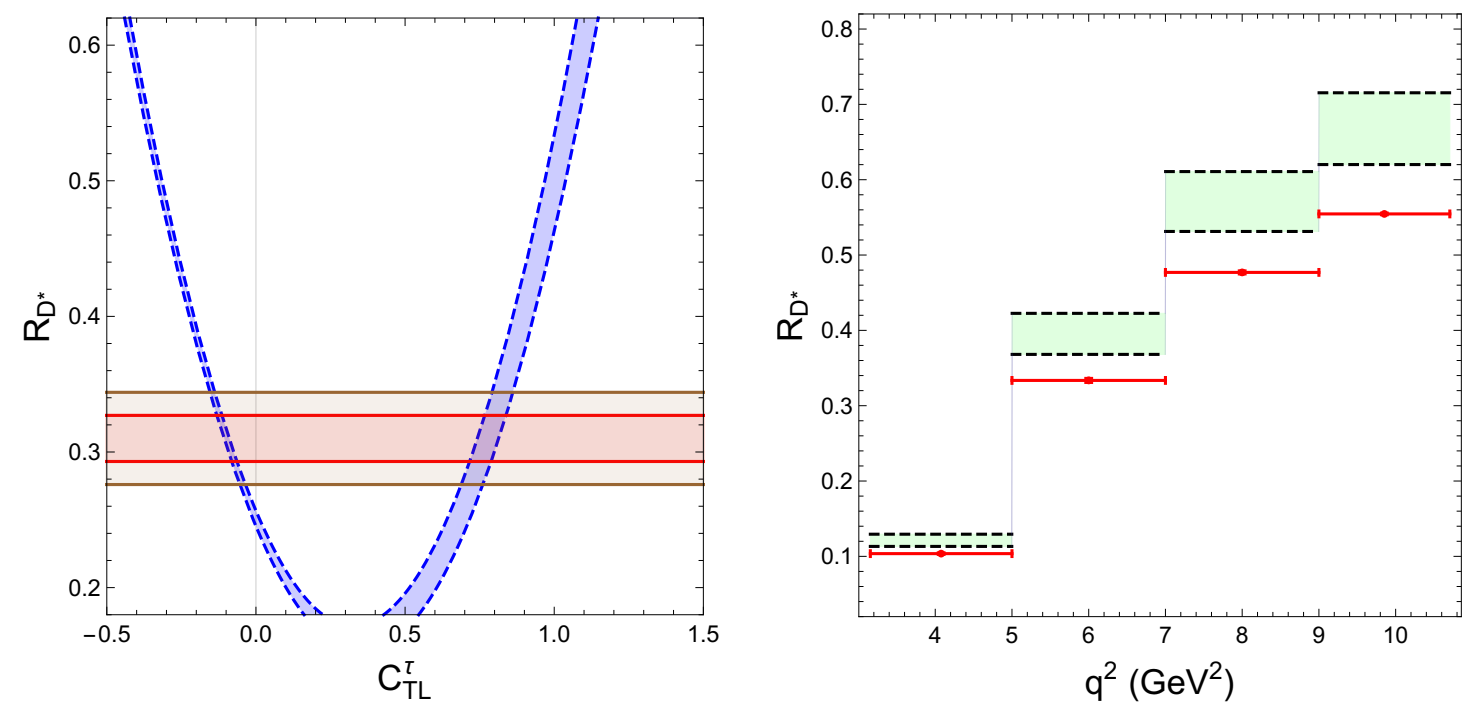

Figure 12. The left panel shows the dependence of $R_{D^{*}}$ with respect to the variation of the WCs $C_{T L}^{\tau}$ and the right panel shows the prediction for $R_{D^{*}}$ in four different bins of $q^{2}$ from table 8 .

\section{C.2 $\bar{B} \rightarrow D^{*} \tau \bar{\nu}_{\tau}$}

The range of $C_{T L}^{\tau}$ that explains $R_{D}^{*}$ within $1 \sigma$ is shown in the first column of table 8 . The resulting values for $P_{\tau}\left(D^{*}\right), \mathcal{A}_{F B}^{D^{*}}$ and binwise $R_{D}^{*}$ are shown in the subsequent columns. In the left plot of figure 12 we also show the dependence of $R_{D}^{*}$ as a function of $C_{T L}^{\tau}$. The right plot shows the binwise $R_{D}^{*}$ graphically.

A quick look at the allowed ranges for $C_{T L}$ in the $B \rightarrow D$ (table 7 ) and the $B \rightarrow D^{*}$ (table 8 ) cases shows that there is a region of overlap, around $0.7-0.8$, which allows one to explain both the anomalies simultaneously.

\section{$\mathrm{D} \quad \mathrm{SU}(3)_{\mathrm{C}} \times \mathrm{SU}(2)_{\mathrm{L}} \times \mathrm{U}(1)_{\mathrm{Y}}$ gauge invariance}

In table 9 we show how the WCs of the operators in this paper are related to the WCs of the gauge invariant dimension 6 operators of [64]. We use the following set of notations:

- Greek letters $\mu, \nu, \cdots$ are used to denote Lorentz indices.

- $\mathrm{SU}(2)$ fundamental indices are denoted by $a, b, \cdots$ and $I, J \cdots$ will be used to denote adjoint indices. 


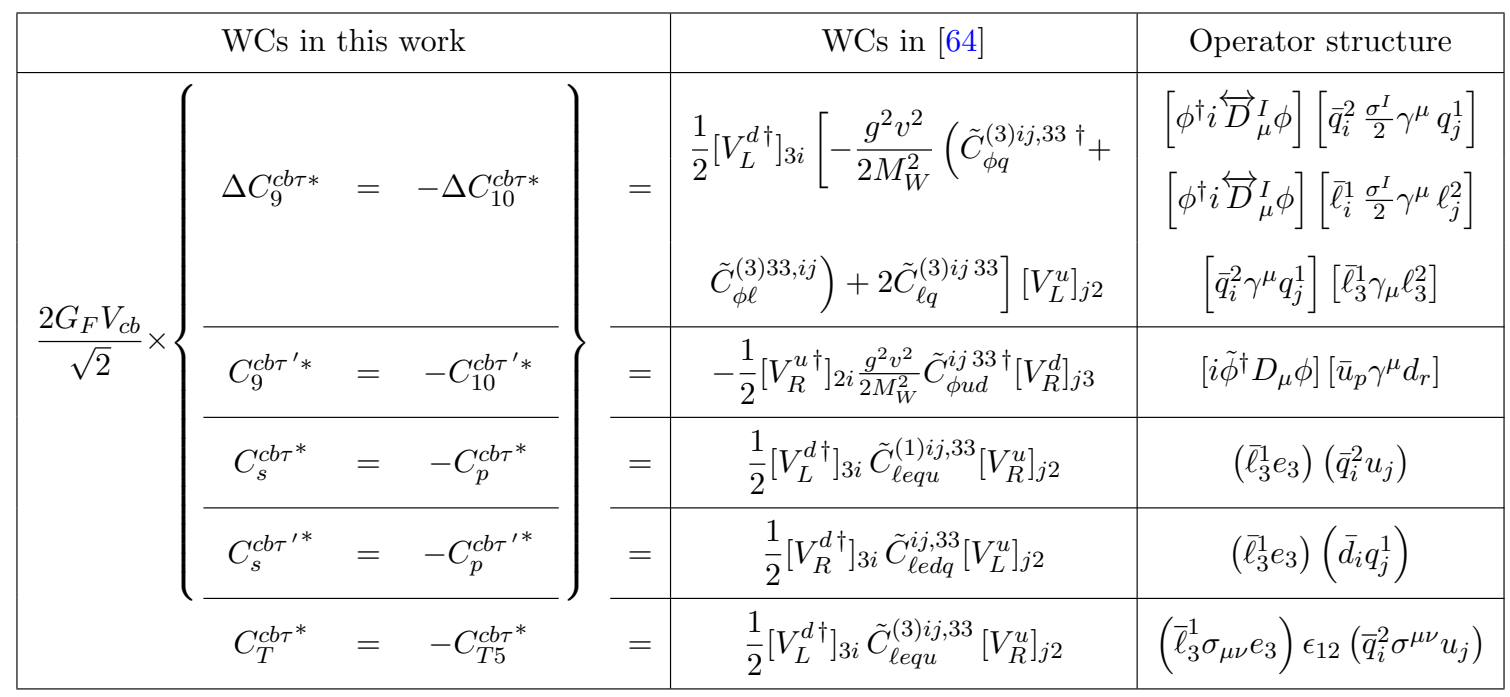

Table 9. Correspondence of our operators with those in reference [64]. The mixing of different lepton flavours are ignored.

- To represent quark (lepton) flavors, we use $i, j, k \cdots(m, n \cdots)$.

- A tilde (e.g. $\tilde{\mathcal{C}}$ ) is used to denote high energy Wilson coefficients.

- The notation for the operators is as given in [64].

- definition of the quark mixing matrices ( $f$ and $m$ denote flavour and mass bases)

$$
\begin{aligned}
u_{L}^{f} & =V_{L}^{u} u_{L}^{m} \\
u_{R}^{f} & =V_{R}^{u} u_{R}^{m} \\
d_{L}^{f} & =V_{L}^{d} d_{L}^{m} \\
d_{R}^{f} & =V_{R}^{d} d_{R}^{m}
\end{aligned}
$$

\section{E RG running of Wilson coefficients}

In this section, we note the renormalisation group (RG) running of the couplings and the Wilson coefficients. The QCD coupling above the $m_{b}$ scale is given by $\alpha_{s}^{(5)}$ and that above the $m_{t}$ scale is given by $\alpha_{s}^{(6)}$. These are given by

$$
\alpha_{s}^{(5)}(\mu)=\frac{\alpha_{s}\left(m_{b}\right)}{1+\beta_{0}^{(5)} \frac{\alpha_{s}\left(m_{b}\right)}{2 \pi} \ln \left(\frac{\mu}{m_{b}}\right)} \quad \alpha_{s}^{(6)}(\mu)=\frac{\alpha_{s}\left(m_{t}\right)}{1+\beta_{0}^{(6)} \frac{\alpha_{s}\left(m_{t}\right)}{2 \pi} \ln \left(\frac{\mu}{m_{t}}\right)}
$$

where $\beta_{0}^{\left(n_{f}\right)}=11-\frac{2 n_{f}}{3}$.

In order to calculate the running of the Wilson Coefficients to a high scale $M$, we need to calculate the beta functions for the different operators - the scalar, vector and tensor operators. The calculation is sketched below (for a good review on the subject, see [69]). 

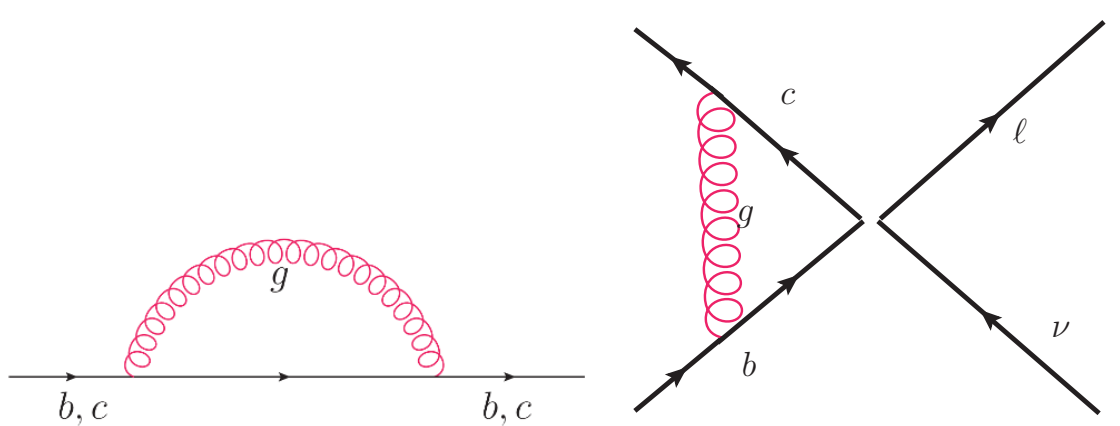

Figure 13. Vertex Correction and self energy diagrams.

Firstly, we need to consider the self-energy correction for the $b$ or $c$ quarks (left diagram in figure 13). This is given by

$$
\begin{aligned}
\Sigma(p) & =i \int \frac{d^{4} k}{(2 \pi)^{4}}\left(i g_{s} \gamma^{\mu} T^{a}\right) \frac{i\left(\not p+\not k+m_{b / c}\right)}{(p+k)^{2}-m_{b / c}^{2}}\left(i g_{s} \gamma^{\nu} T^{b}\right) \frac{\left(-i g_{\mu \nu} \delta_{a b}\right)}{k^{2}} \\
& =\frac{4}{3}(-\frac{\alpha_{s}}{4 \pi} \not p+\underbrace{\frac{\alpha_{s} m_{b / c}}{\pi}}_{\text {dropped }}) \frac{1}{\epsilon}+\text { finite }
\end{aligned}
$$

where $p$ is the momentum of the incoming (or outgoing) quark.

From Feynman diagram on the right of figure 13, we find that the vertex correction in $d$ dimensions $(d=4-2 \epsilon)$ is given by

$$
\begin{aligned}
\Gamma_{\text {Had }}\left(p, p^{\prime}\right) & =i \int \frac{d^{d} k}{(2 \pi)^{d}}\left(i g_{s} \gamma^{\lambda} T^{a}\right) \frac{i}{\not p+\not k-m_{b}} i \mathcal{F} \frac{i}{\not p^{\prime}+\not k-m_{c}}\left(i g_{s} \gamma^{\sigma} T^{b}\right)\left(-i \delta_{a b} g_{\lambda \sigma}\right) \frac{1}{k^{2}} \\
& =i g_{s}^{2} C_{2}(3) \int \frac{d^{d} k}{(2 \pi)^{d}} \frac{\gamma_{\lambda}\left(\not p+\not k+m_{b}\right) \mathcal{F}\left(\not p^{\prime}+\not k+m_{c}\right) \gamma^{\lambda}}{k^{2}\left((p+k)^{2}+m_{b}^{2}\right)\left(\left(p^{\prime}+k\right)^{2}+m_{c}^{2}\right)}
\end{aligned}
$$

where $C_{2}(3)=\frac{4}{3}$ and $\mathcal{F}=1, \gamma_{\mu}, \sigma_{\mu \nu}$ for scalar, vector and tensor operators and $p\left(p^{\prime}\right)$ is the on-shell momentum of the $b(c)$ quark. A few things are noteworthy and enlisted below:

- As the denominator has mass dimension 6, divergence will appear only when the numerator is a function of loop momentum with mass dimension greater than and equals to two.

- The general form of the numerator is

$$
\begin{aligned}
N & =\gamma_{\lambda}\left(\not{ }^{\prime}+\not k+m_{b}\right) \mathcal{F}\left(\not p+\not k+m_{c}\right) \gamma^{\lambda} \\
& =\gamma_{\lambda} \not k \mathcal{F} \not k \gamma^{\lambda}+\text { finite }
\end{aligned}
$$

- For scalar

$$
N=4 k^{2}
$$


- For vector

$$
N=\gamma_{\lambda} k \gamma_{\mu} k \gamma^{\lambda}=-k^{2} \gamma_{\lambda} \gamma_{\mu} \gamma^{\lambda}+2 k_{\mu} \gamma_{\lambda} k \gamma^{\lambda}=2 k^{2} \gamma_{\mu}-4 k_{\mu} k
$$

Using

$$
\int d^{4} k k^{\mu} k^{\nu} f\left(k^{2}\right)=\frac{1}{4} g^{\mu \nu} \int d^{4} k k^{2} f\left(k^{2}\right)
$$

we get

$$
N=k^{2} \gamma_{\mu}
$$

- For tensor

$$
N=\gamma_{\lambda} k \sigma_{\mu \nu} k \gamma^{\lambda} \longmapsto k^{2} \frac{1}{4} \gamma_{\lambda} \gamma_{\rho} \sigma_{\mu \nu} \gamma^{\rho} \gamma^{\lambda}=0
$$

where we used the previous integral formula in the second step.

Putting this back and using Feynman parameterisation and neglecting quark masses, we have the following formula

$$
\begin{aligned}
\Gamma_{\text {Had }} & =i g_{s}^{2} C_{2}(3) \mathcal{N F} \int_{0}^{1} d \zeta \int \frac{d^{d} k}{(2 \pi)^{d}} \frac{1}{\left[\zeta(p+k)^{2}+(1-\zeta)\left(p^{\prime}+k\right)^{2}\right]^{2}} \\
& =i \frac{16 \pi}{3} \alpha_{s} \mathcal{N} \mathcal{F} \int_{0}^{1} d \zeta \int \frac{d^{d} \ell}{(2 \pi)^{d}} \frac{1}{\left(\ell^{2}+\Delta\right)^{2}} \\
& \text { where } \ell=k+p+(1-\zeta)\left(p^{\prime}-p\right) \text { and } \Delta=\zeta(1-\zeta)\left(p^{\prime}-p\right)^{2} \\
& =i \frac{16 \pi}{3} \alpha_{s} \mathcal{N} \mathcal{F} \int_{0}^{1} d \zeta \frac{i}{(4 \pi)^{2}}\left(\frac{2}{\epsilon}+\text { finite }\right) \\
& =-\frac{\alpha_{s}}{4 \pi} \frac{8 \mathcal{N}}{3} \mathcal{F} \frac{1}{\epsilon}+\text { finite }
\end{aligned}
$$

where $\mathcal{N}=4,1,0$ for $\mathcal{F}=1, \gamma^{\mu}, \sigma^{\mu \nu}$ respectively. The bare effective Lagrangian to the lowest power in derivatives is

$$
\mathcal{L}_{\text {eff }}^{\text {bare }}=i \bar{\psi}_{0} \not \partial \psi_{0}+\mathcal{C} \bar{c}_{0} \mathcal{F} b_{0} \bar{\ell}_{0} \mathcal{F}^{\prime} \nu_{\ell 0}
$$

where $\psi_{0}$ is any bare quark or lepton field, $\mathcal{C}$ is the Wilson coefficient to the six-dimensional operator and $\mathcal{F}, \mathcal{F}^{\prime}$ are Dirac operators.

We redefine the quantities in the bare Lagrangian as

$$
\psi_{0}=\sqrt{Z_{\psi}} \psi ; \quad \mathcal{C}_{0}=\mu^{2 \epsilon} Z_{\mathcal{C}} \mathcal{C}
$$

where $\psi$ represents any quark field. The QCD contributions to the different quark fields will be equal to each other. Then eq. (E.9) can then be written as

$$
\begin{aligned}
\mathcal{L}_{\text {eff }}^{\mathrm{ren}} & =i Z_{\psi} \bar{\psi} \not \partial \psi+\mathcal{C} Z_{\mathcal{C}} Z_{\psi}^{2} \mu^{2 \epsilon} \bar{c} \mathcal{F} b \bar{\ell} \mathcal{F}^{\prime} \nu_{\ell} \\
& =i \bar{\psi} \not \partial \psi+i\left(Z_{\psi}-1\right) \bar{\psi} \not \partial \psi+\mathcal{C} \mu^{2 \epsilon} \bar{c} \mathcal{F} b \bar{\ell} \mathcal{F}^{\prime} \nu_{\ell}+\mathcal{C}\left(Z_{\mathcal{C}} Z_{\psi}^{2}-1\right) \mu^{2 \epsilon} \bar{c} \mathcal{F} b \bar{\ell} \mathcal{F}^{\prime} \nu_{\ell}
\end{aligned}
$$

Absorbing the divergences in eq. (E.2) and eq. (E.8) in the counter terms, we find that

$$
Z_{\psi}=1-\frac{4}{3} \frac{\alpha_{s}}{4 \pi} \frac{1}{\epsilon} \text { and } Z_{\mathcal{C}}=1-\frac{8}{3} \frac{\alpha_{s}}{4 \pi}(\mathcal{N}-1) \frac{1}{\epsilon}
$$



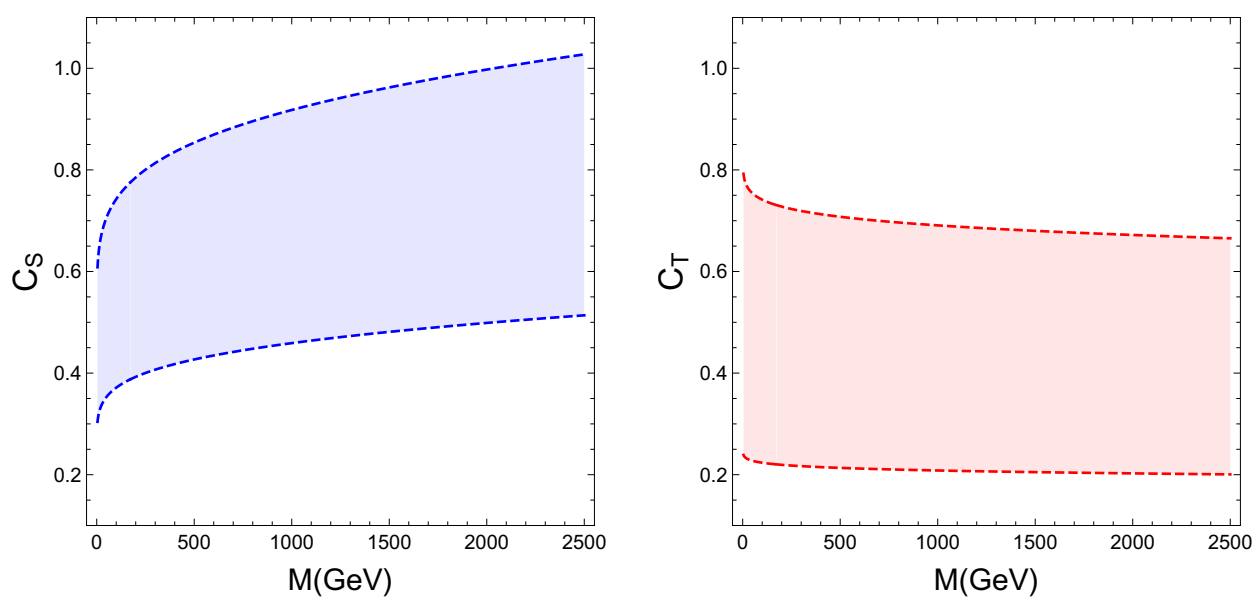

Figure 14. Plot of the running of the Scalar (left) and Tensor (right) Wilson Coefficients. The range of the running is from $m_{b}$ to $2.5 \mathrm{TeV}$. As a demonstration, the range of the initial values used are the ones mentioned in the text for $B \rightarrow D$ decay.

Using the RG equations, the $\beta$-function turns out to be

$$
\begin{aligned}
\beta_{\mathcal{C}} & =-2 \epsilon \mathcal{C}-\frac{\mu}{Z_{\mathcal{C}}} \mathcal{C} \frac{d Z_{\mathcal{C}}}{d \mu} \\
& =\frac{8}{3} \frac{1}{4 \pi}(\mathcal{N}-1) \mathcal{C} \frac{\mu}{Z_{\mathcal{C}}} \frac{d \alpha_{s}}{d \mu} \frac{1}{\epsilon} \\
& =-\frac{8}{3} \frac{\alpha_{s}}{4 \pi}(\mathcal{N}-1) \mathcal{C}
\end{aligned}
$$

Thus,

$$
\beta_{\mathcal{C}}^{S}=-\mathbf{8} \frac{\alpha_{s}}{4 \pi} \mathcal{C}, \beta_{\mathcal{C}}^{V}=\mathbf{0}, \text { and } \beta_{\mathcal{C}}^{T}=\frac{\mathbf{8}}{\mathbf{3}} \frac{\alpha_{s}}{4 \pi} \mathcal{C}
$$

where the superscripts $S, V$ and $T$ on the $\beta$ denote scalar, vector and tensor couplings. The running of the Wilson Coefficients can be found by solving the $\beta$-function equation given in eq. (E.13). Solving, we get,

$$
\tilde{C}\left(m_{b}\right)=\left[\frac{\alpha_{s}\left(m_{t}\right)}{\alpha_{s}\left(m_{b}\right)}\right]^{\frac{\gamma}{2 \beta_{0}^{(5)}}}\left[\frac{\alpha_{s}(M)}{\alpha_{s}\left(m_{t}\right)}\right]^{\frac{\gamma}{2 \beta_{0}^{(6)}}} \tilde{C}(M)
$$

Thus, the scalar and tensor WCs are given by:

$$
\begin{aligned}
& \tilde{C}_{S}(M)=\left[\left[\frac{\alpha_{s}\left(m_{t}\right)}{\alpha_{s}\left(m_{b}\right)}\right]^{\frac{\gamma_{S}}{2 \beta_{0}^{(5)}}}\left[\frac{\alpha_{s}(M)}{\alpha_{s}\left(m_{t}\right)}\right]^{\frac{\gamma_{S}}{2 \beta_{0}^{(6)}}}\right]^{-1} \tilde{C}_{S}\left(m_{b}\right) \\
& \tilde{C}_{T}(M)=\left[\left[\frac{\alpha_{s}\left(m_{t}\right)}{\alpha_{s}\left(m_{b}\right)}\right]^{\frac{\gamma_{T}}{2 \beta_{0}^{(5)}}}\left[\frac{\alpha_{s}(M)}{\alpha_{s}\left(m_{t}\right)}\right]^{\frac{\gamma_{T}}{2 \beta_{0}^{(6)}}}\right]^{-1} \tilde{C}_{T}\left(m_{b}\right)
\end{aligned}
$$

where

$$
\gamma_{S}=-8 \quad \gamma_{T}=\frac{8}{3}
$$

which are simply the boldfaced coefficients in eq. (E.13). This is plotted in figure 14 . 
Open Access. This article is distributed under the terms of the Creative Commons Attribution License (CC-BY 4.0), which permits any use, distribution and reproduction in any medium, provided the original author(s) and source are credited.

\section{References}

[1] LHCb collaboration, Test of lepton universality using $B^{+} \rightarrow K^{+} \ell^{+} \ell^{-}$decays, Phys. Rev. Lett. 113 (2014) 151601 [arXiv:1406.6482] [INSPIRE].

[2] LHCb collaboration, Angular analysis of the $B^{0} \rightarrow K^{* 0} \mu^{+} \mu^{-}$decay using $3 \mathrm{fb}^{-1}$ of integrated luminosity, JHEP 02 (2016) 104 [arXiv:1512.04442] [INSPIRE].

[3] W. Altmannshofer, P. Ball, A. Bharucha, A.J. Buras, D.M. Straub and M. Wick, Symmetries and Asymmetries of $B \rightarrow K^{*} \mu^{+} \mu^{-}$Decays in the Standard Model and Beyond, JHEP 01 (2009) 019 [arXiv:0811.1214] [INSPIRE].

[4] A.K. Alok et al., New-physics contributions to the forward-backward asymmetry in $B \rightarrow K^{*} \mu^{+} \mu^{-}$, JHEP 02 (2010) 053 [arXiv:0912.1382] [INSPIRE].

[5] A.K. Alok, A. Datta, A. Dighe, M. Duraisamy, D. Ghosh and D. London, New Physics in $b \rightarrow s \mu^{+} \mu^{-}$: CP-Conserving Observables, JHEP 11 (2011) 121 [arXiv:1008.2367] [INSPIRE].

[6] A.K. Alok, A. Datta, A. Dighe, M. Duraisamy, D. Ghosh and D. London, New Physics in $b \rightarrow s \mu^{+} \mu^{-}$: CP-Violating Observables, JHEP 11 (2011) 122 [arXiv:1103.5344] [INSPIRE].

[7] S. Descotes-Genon, D. Ghosh, J. Matias and M. Ramon, Exploring New Physics in the C7-C7' plane, JHEP 06 (2011) 099 [arXiv:1104.3342] [INSPIRE].

[8] S. Descotes-Genon, J. Matias and J. Virto, Understanding the $B \rightarrow K^{*} \mu^{+} \mu^{-}$Anomaly, Phys. Rev. D 88 (2013) 074002 [arXiv:1307.5683] [INSPIRE].

[9] W. Altmannshofer and D.M. Straub, New Physics in $B \rightarrow K^{*} \mu \mu$ ?, Eur. Phys. J. C 73 (2013) 2646 [arXiv: 1308.1501] [inSPIRE].

[10] A. Datta, M. Duraisamy and D. Ghosh, Explaining the $B \rightarrow K^{*} \mu^{+} \mu^{-}$data with scalar interactions, Phys. Rev. D 89 (2014) 071501 [arXiv:1310.1937] [InSPIRE].

[11] D. Ghosh, M. Nardecchia and S.A. Renner, Hint of Lepton Flavour Non-Universality in $B$ Meson Decays, JHEP 12 (2014) 131 [arXiv:1408.4097] [INSPIRE].

[12] R. Mandal, R. Sinha and D. Das, Testing New Physics Effects in $B \rightarrow K^{*} \ell^{+} \ell^{-}$, Phys. Rev. D 90 (2014) 096006 [arXiv: 1409.3088] [INSPIRE].

[13] W. Altmannshofer and D.M. Straub, New physics in $b \rightarrow s$ transitions after LHC run 1, Eur. Phys. J. C 75 (2015) 382 [arXiv:1411.3161] [InSPIRE].

[14] S. Jäger and J. Martin Camalich, Reassessing the discovery potential of the $B \rightarrow K^{*} \ell^{+} \ell^{-}$ decays in the large-recoil region: SM challenges and BSM opportunities, Phys. Rev. D 93 (2016) 014028 [arXiv:1412.3183] [INSPIRE].

[15] S. Descotes-Genon, L. Hofer, J. Matias and J. Virto, Global analysis of $b \rightarrow$ sll anomalies, JHEP 06 (2016) 092 [arXiv: 1510.04239] [INSPIRE].

[16] M. Ciuchini et al., $B \rightarrow K^{*} \ell^{+} \ell^{-}$decays at large recoil in the Standard Model: a theoretical reappraisal, JHEP 06 (2016) 116 [arXiv:1512.07157] [INSPIRE]. 
[17] Heavy Flavor Averaging Group (HFAG) collaboration, Y. Amhis et al., Averages of b-hadron, c-hadron and $\tau$-lepton properties as of summer 2014, arXiv:1412.7515 [INSPIRE].

[18] BeLle collaboration, M. Huschle et al., Measurement of the branching ratio of $\bar{B} \rightarrow D^{(*)} \tau^{-} \bar{\nu}_{\tau}$ relative to $\bar{B} \rightarrow D^{(*)} \ell^{-} \bar{\nu}_{\ell}$ decays with hadronic tagging at Belle, Phys. Rev. $\mathbf{D}$ 92 (2015) 072014 [arXiv: 1507.03233] [INSPIRE].

[19] MILC collaboration, J.A. Bailey et al., $B \rightarrow D \ell \nu$ form factors at nonzero recoil and $\left|V_{c b}\right|$ from 2+1-flavor lattice QCD, Phys. Rev. D 92 (2015) 034506 [arXiv:1503.07237] [INSPIRE].

[20] BABAR collaboration, J.P. Lees et al., Evidence for an excess of $\bar{B} \rightarrow D^{(*)} \tau^{-} \bar{\nu}_{\tau}$ decays, Phys. Rev. Lett. 109 (2012) 101802 [arXiv:1205.5442] [INSPIRE].

[21] BABAR collaboration, J.P. Lees et al., Measurement of an Excess of $\bar{B} \rightarrow D^{(*)} \tau^{-} \bar{\nu}_{\tau}$ Decays and Implications for Charged Higgs Bosons, Phys. Rev. D 88 (2013) 072012 [arXiv: 1303.0571] [INSPIRE].

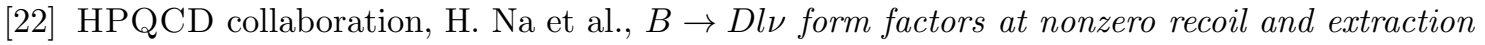
of $\left|V_{c b}\right|$, Phys. Rev. D 92 (2015) 054510 [arXiv: 1505.03925] [inSPIRE].

[23] D. Bigi and P. Gambino, Revisiting B $\rightarrow$ D $\ell \nu$, Phys. Rev. D 94 (2016) 094008 [arXiv:1606.08030] [INSPIRE].

[24] S. Fajfer, J.F. Kamenik and I. Nisandzic, On the $B \rightarrow D^{*} \tau \bar{\nu}_{\tau}$ Sensitivity to New Physics, Phys. Rev. D 85 (2012) 094025 [arXiv: 1203.2654] [INSPIRE].

[25] BeLLe collaboration, A. Abdesselam et al., Measurement of the branching ratio of $\bar{B}^{0} \rightarrow D^{*+} \tau^{-} \bar{\nu}_{\tau}$ relative to $\bar{B}^{0} \rightarrow D^{*+} \ell^{-} \bar{\nu}_{\ell}$ decays with a semileptonic tagging method, arXiv: 1603.06711 [INSPIRE].

[26] LHCb collaboration, Measurement of the ratio of branching fractions $\mathcal{B}\left(\bar{B}^{0} \rightarrow D^{*+} \tau^{-} \bar{\nu}_{\tau}\right) / \mathcal{B}\left(\bar{B}^{0} \rightarrow D^{*+} \mu^{-} \bar{\nu}_{\mu}\right)$, Phys. Rev. Lett. 115 (2015) 111803 [arXiv: 1506.08614] [INSPIRE].

[27] A. Abdesselam et al., Measurement of the $\tau$ lepton polarization in the decay $\bar{B} \rightarrow D^{*} \tau^{-} \bar{\nu}_{\tau}$, arXiv:1608.06391 [INSPIRE].

[28] M. Tanaka and R. Watanabe, Tau longitudinal polarization in $B \rightarrow D \tau \nu$ and its role in the search for charged Higgs boson, Phys. Rev. D 82 (2010) 034027 [arXiv: 1005.4306] [INSPIRE].

[29] M. Tanaka and R. Watanabe, New physics in the weak interaction of $\bar{B} \rightarrow D^{(*)} \tau \bar{\nu}$, Phys. Rev. D 87 (2013) 034028 [arXiv:1212.1878] [INSPIRE].

[30] U. Nierste, S. Trine and S. Westhoff, Charged-Higgs effects in a new $B \rightarrow D \tau \nu$ differential decay distribution, Phys. Rev. D 78 (2008) 015006 [arXiv:0801.4938] [InSPIRE].

[31] A. Datta, M. Duraisamy and D. Ghosh, Diagnosing New Physics in $b \rightarrow c \tau \nu_{\tau}$ decays in the light of the recent BaBar result, Phys. Rev. D 86 (2012) 034027 [arXiv:1206.3760] [INSPIRE].

[32] Y. Sakaki and H. Tanaka, Constraints on the charged scalar effects using the forward-backward asymmetry on $B \rightarrow D^{*} \tau \nu$, Phys. Rev. D 87 (2013) 054002 [arXiv: 1205.4908] [INSPIRE].

[33] A. Crivellin, C. Greub and A. Kokulu, Explaining $B \rightarrow D \tau \nu, B \rightarrow D^{*} \tau \nu$ and $B \rightarrow \tau \nu$ in a 2HDM of type-III, Phys. Rev. D 86 (2012) 054014 [arXiv:1206.2634] [InSPIRE]. 
[34] D. Choudhury, D.K. Ghosh and A. Kundu, B decay anomalies in an effective theory, Phys. Rev. D 86 (2012) 114037 [arXiv:1210.5076] [INSPIRE].

[35] A. Celis, M. Jung, X.-Q. Li and A. Pich, Sensitivity to charged scalars in $B \rightarrow D^{(*)} \tau \nu_{\tau}$ and $B \rightarrow \tau \nu_{\tau}$ decays, JHEP 01 (2013) 054 [arXiv: 1210.8443] [INSPIRE].

[36] Y. Sakaki, M. Tanaka, A. Tayduganov and R. Watanabe, Testing leptoquark models in $\bar{B} \rightarrow D^{(*)} \tau \bar{\nu}$, Phys. Rev. D 88 (2013) 094012 [arXiv:1309.0301] [InSPIRE].

[37] I. Doršner, S. Fajfer, N. Košnik and I. Nišandžić, Minimally flavored colored scalar in $\bar{B} \rightarrow D^{(*)} \tau \bar{\nu}$ and the mass matrices constraints, JHEP 11 (2013) 084 [arXiv:1306.6493] [INSPIRE].

[38] M. Duraisamy and A. Datta, The Full $B \rightarrow D^{*} \tau^{-} \overline{\nu_{\tau}}$ Angular Distribution and CP-violating Triple Products, JHEP 09 (2013) 059 [arXiv: 1302.7031] [INSPIRE].

[39] P. Biancofiore, P. Colangelo and F. De Fazio, On the anomalous enhancement observed in $B \rightarrow D^{(*)} \tau \bar{\nu}_{\tau}$ decays, Phys. Rev. D 87 (2013) 074010 [arXiv:1302.1042] [INSPIRE].

[40] M. Duraisamy, P. Sharma and A. Datta, Azimuthal $B \rightarrow D^{*} \tau^{-} \overline{\nu_{\tau}}$ angular distribution with tensor operators, Phys. Rev. D 90 (2014) 074013 [arXiv: 1405.3719] [INSPIRE].

[41] M. Freytsis, Z. Ligeti and J.T. Ruderman, Flavor models for $\bar{B} \rightarrow D^{(*)} \tau \bar{\nu}$, Phys. Rev. D 92 (2015) 054018 [arXiv: 1506. 08896] [INSPIRE].

[42] A. Greljo, G. Isidori and D. Marzocca, On the breaking of Lepton Flavor Universality in B decays, JHEP 07 (2015) 142 [arXiv: 1506.01705] [INSPIRE].

[43] L. Calibbi, A. Crivellin and T. Ota, Effective Field Theory Approach to $b \rightarrow$ sl( $^{(\prime)}$, $B \rightarrow K^{(*)} \nu \bar{\nu}$ and $B \rightarrow D^{(*)} \tau \nu$ with Third Generation Couplings, Phys. Rev. Lett. 115 (2015) 181801 [arXiv: 1506.02661] [INSPIRE].

[44] S. Bhattacharya, S. Nandi and S.K. Patra, Optimal-observable analysis of possible new physics in $B \rightarrow D^{(*)} \tau \nu_{\tau}$, Phys. Rev. D 93 (2016) 034011 [arXiv: 1509.07259] [INSPIRE].

[45] C. Hati, G. Kumar and N. Mahajan, $\bar{B} \rightarrow D^{(*)} \tau \bar{\nu}$ excesses in ALRSM constrained from $B$, $D$ decays and $D^{0}-\bar{D}^{0}$ mixing, JHEP 01 (2016) 117 [arXiv:1511.03290] [INSPIRE].

[46] M. Bauer and M. Neubert, Minimal Leptoquark Explanation for the $R_{D^{(*)}}, R_{K}$ and $(g-2)_{g}$ Anomalies, Phys. Rev. Lett. 116 (2016) 141802 [arXiv:1511.01900] [INSPIRE].

[47] R. Barbieri, G. Isidori, A. Pattori and F. Senia, Anomalies in B-decays and U(2) flavour symmetry, Eur. Phys. J. C 76 (2016) 67 [arXiv:1512.01560] [INSPIRE].

[48] M.A. Ivanov, J.G. Körner and C.T. Tran, Exclusive decays $B \rightarrow \ell^{-} \bar{\nu}$ and $B \rightarrow D^{(*)} \ell^{-} \bar{\nu}$ in the covariant quark model, Phys. Rev. D 92 (2015) 114022 [arXiv:1508. 02678] [InSPIRE].

[49] J.M. Cline, Scalar doublet models confront $\tau$ and $b$ anomalies, Phys. Rev. D 93 (2016) 075017 [arXiv: 1512.02210] [INSPIRE].

[50] D. Das, C. Hati, G. Kumar and N. Mahajan, Towards a unified explanation of $R_{D^{(*)}}, R_{K}$ and $(g-2)_{\mu}$ anomalies in a left-right model with leptoquarks, Phys. Rev. D 94 (2016) 055034 [arXiv: 1605.06313] [INSPIRE].

[51] M. Bordone, G. Isidori and D. van Dyk, Impact of leptonic $\tau$ decays on the distribution of $B \rightarrow P \mu \bar{\nu}$ decays, Eur. Phys. J. C 76 (2016) 360 [arXiv: 1602.06143] [InSPIRE].

[52] R. Alonso, A. Kobach and J. Martin Camalich, New physics in the kinematic distributions of $\bar{B} \rightarrow D^{(*)} \tau^{-}\left(\rightarrow \ell^{-} \bar{\nu}_{\ell} \nu_{\tau}\right) \bar{\nu}_{\tau}$, Phys. Rev. D 94 (2016) 094021 [arXiv:1602.07671] [inSPIRE]. 
[53] S. Nandi, S.K. Patra and A. Soni, Correlating new physics signals in $B \rightarrow D^{(*)} \tau \nu_{\tau}$ with $B \rightarrow \tau \nu_{\tau}$, arXiv:1605.07191 [INSPIRE].

[54] F. Feruglio, P. Paradisi and A. Pattori, Revisiting Lepton Flavour Universality in B Decays, Phys. Rev. Lett. 118 (2017) 011801 [arXiv:1606.00524] [INSPIRE].

[55] A.K. Alok, D. Kumar, S. Kumbhakar and S.U. Sankar, $D^{*}$ polarization as a probe to discriminate new physics in $B \rightarrow D^{*} \tau \bar{\nu}$, arXiv:1606.03164 [INSPIRE].

[56] S.M. Boucenna, A. Celis, J. Fuentes-Martin, A. Vicente and J. Virto, Non-abelian gauge extensions for B-decay anomalies, Phys. Lett. B 760 (2016) 214 [arXiv:1604.03088] [INSPIRE].

[57] S.M. Boucenna, A. Celis, J. Fuentes-Martin, A. Vicente and J. Virto, Phenomenology of an $\mathrm{SU}(2) \times \mathrm{SU}(2) \times \mathrm{U}(1)$ model with lepton-flavour non-universality, JHEP 12 (2016) 059 [arXiv: 1608.01349] [INSPIRE].

[58] S. Sahoo, R. Mohanta and A.K. Giri, Explaining $R_{K}$ and $R_{D^{(*)}}$ anomalies with vector leptoquark, arXiv: 1609.04367 [INSPIRE].

[59] D.A. Faroughy, A. Greljo and J.F. Kamenik, Confronting lepton flavor universality violation in B decays with high-p $p_{T}$ tau lepton searches at LHC, Phys. Lett. B 764 (2017) 126 [arXiv: 1609.07138] [INSPIRE].

[60] Z. Ligeti, M. Papucci and D.J. Robinson, New Physics in the Visible Final States of $B \rightarrow D^{(*)} \tau \nu$, JHEP 01 (2017) 083 [arXiv:1610.02045] [inSPIRE].

[61] M.A. Ivanov, J.G. Körner and C.-T. Tran, Analyzing new physics in the decays $\bar{B}^{0} \rightarrow D^{(*)} \tau^{-} \bar{\nu}_{\tau}$ with form factors obtained from the covariant quark model, Phys. Rev. D 94 (2016) 094028 [arXiv: 1607.02932] [INSPIRE].

[62] I. Doršner, S. Fajfer, A. Greljo, J.F. Kamenik and N. Košnik, Physics of leptoquarks in precision experiments and at particle colliders, Phys. Rept. 641 (2016) 1 [arXiv:1603.04993] [INSPIRE].

[63] D. Bečirević, S. Fajfer, N. Košnik and O. Sumensari, Leptoquark model to explain the B-physics anomalies, $R_{K}$ and $R_{D}$, Phys. Rev. D 94 (2016) 115021 [arXiv:1608.08501] [INSPIRE].

[64] B. Grzadkowski, M. Iskrzynski, M. Misiak and J. Rosiek, Dimension-Six Terms in the Standard Model Lagrangian, JHEP 10 (2010) 085 [arXiv: 1008.4884] [INSPIRE].

[65] D. Melikhov and B. Stech, Weak form-factors for heavy meson decays: An update, Phys. Rev. D 62 (2000) 014006 [hep-ph/0001113] [INSPIRE].

[66] I. Caprini, L. Lellouch and M. Neubert, Dispersive bounds on the shape of $\bar{B} \rightarrow D^{*} \ell \bar{\nu}$ form-factors, Nucl. Phys. B 530 (1998) 153 [hep-ph/9712417] [INSPIRE].

[67] Fermilab Lattice, MiLC collaborations, J.A. Bailey et al., Update of $\left|V_{c b}\right|$ from the $\bar{B} \rightarrow D^{*} \ell \bar{\nu}$ form factor at zero recoil with three-flavor lattice QCD, Phys. Rev. D 89 (2014) 114504 [arXiv: 1403.0635] [INSPIRE].

[68] B. Golob, Belle II, talk given at Flavour Physics with High-Luminosity Experiments, Munich, Germany (2016), http://indico.ijs.si/getFile.py/access? contribId $=1 \&$ resId $=0 \&$ materialId $=$ slides\&confId $=801$.

[69] W. Skiba, Effective Field Theory and Precision Electroweak Measurements, arXiv:1006.2142 [INSPIRE]. 